\title{
CBZ6 as a Recyclable Organic Photoreductant for Pinacol Coupling
}

\author{
Hua Wang, ${ }^{\dagger}$ Jian-Ping Qu, ${ }^{*}$ and Yan-Biao Kang* ${ }^{\dagger}$ \\ ${ }^{\dagger}$ Department of Chemistry, University of Science and Technology of China, Hefei, Anhui 230026, China \\ ${ }^{\ddagger}$ Institute of Advanced Synthesis, School of Chemistry and Molecular Engineering, Jiangsu National \\ Synergetic Innovation Center for Advanced Materials, Nanjing Tech University, Nanjing 211816, China \\ E-mail: ybkang@ustc.edu.cn
}

Table of Contents

1. General Information

S2

2. Experimental Procedures

S2-S14

3. Fluorescence and Luminescence Experiments

S15

4. References

S15

5. NMR spectra

S16-S95 


\section{General Information}

All reactions were carried out under atmospheric pressure. Solvents were pre-dried over activated $4 \AA$ molecular sieves and heated to reflux over calcium hydride or Mg turnings and iodine crystals $\left(\mathrm{PhCN}, \mathrm{CH}_{3} \mathrm{CN}, \mathrm{DCM}, \mathrm{Et}_{3} \mathrm{~N}, \mathrm{THF}, \mathrm{DMF}, \mathrm{PhCl}, \mathrm{DMSO}, \mathrm{MeOH}\right)$ under argon atmosphere and collected by distillation. Aldehydes were used with purification as commercially available. Aldehydes, ketones, imines and other chemicals without notes in experimental section were purchased from commercial sources. All reactions were performed with SemiLEDs lamps (C35LU-60), the glass reaction tube was placed $5 \mathrm{~cm}$ away from LEDs. All reactions were monitored by thin layer chromatography. Purification of reaction products were carried out by flash chromatography on silica gel or aluminum oxide active neutral. Chemical yields refer to pure isolated substances. All work-up and purification procedures were carried out with reagent-grade solvents in air. ${ }^{1} \mathrm{H},{ }^{19} \mathrm{~F}$ decoupled, ${ }^{13} \mathrm{C}\left\{{ }^{1} \mathrm{H}\right\}$ NMR spectra were recorded on a Bruker $400 / 500$ spectrometer; Chemical shifts are reported in $\delta$ units relative to $\mathrm{CDCl}_{3}\left[{ }^{1} \mathrm{H} \delta=7.26,{ }^{13} \mathrm{C} \delta=77.16\right]$ and $d^{6}$-DMSO $\left[{ }^{1} \mathrm{H} \delta=2.50,{ }^{13} \mathrm{C} \delta=39.52\right]$. High resolution mass spectral analysis (HRMS) was performed on Waters XEVO G2 Q-TOF (Waters Corporation). Fluorescence Spectrum was recorded on an F-4600 spectrometer.

\section{Experimental Procedures}

\subsection{Optimization of Reaction Conditions.}

Procedure for reductive coupling of aldehydes: CBZ6 (0.002 mmol, $1 \mathrm{~mol} \%)$ was added in an oven-dried $25 \mathrm{~mL}$ Schlenk tube. The reaction tube was placed under vacuum and backfilled with argon three times. Then aldehydes ( $0.2 \mathrm{mmol}, 1$ equiv), amine and dry solvent $(2 \mathrm{~mL})$ were added sequentially in the Schlenk tube via syringe. Then the tube was placed $5 \mathrm{~cm}$ away from LEDs $(3 \mathrm{~W} \times 3)$, and stirred vigorously under the irradiation of light. After $2.5 \mathrm{~h}$, the tube was removed from the light source, the reaction mixture was diluted with $\mathrm{CH}_{2} \mathrm{Cl}_{2}(2 \mathrm{~mL})$, and the product yield was determined by ${ }^{1} \mathrm{H}$ NMR technology using nitromethane and tert-butyl methyl ether as internal standard.

\section{Table S1. Screening Reaction Conditions ${ }^{a}$}

\begin{tabular}{|c|c|c|c|c|c|c|}
\hline Entry & PC & amine & additive & solvent & $\mathbf{2 a}(\%)^{b}$ & meso/dl \\
\hline 1 & CBZ6 & DIPEA ( 2 equiv) & - & $\mathrm{MeCN}$ & 75 & $1: 1.1$ \\
\hline 2 & CBZ6 & DIPEA (1 equiv) & - & $\mathrm{MeCN}$ & 75 & $1: 1.1$ \\
\hline 3 & CBZ6 & $\mathrm{Et}_{3} \mathrm{~N}$ & - & $\mathrm{MeCN}$ & 75 & $1: 1.1$ \\
\hline 4 & CBZ6 & DABCO & - & $\mathrm{MeCN}$ & 0 & - \\
\hline 5 & CBZ6 & DIPA & - & $\mathrm{MeCN}$ & 30 & $1: 1$ \\
\hline 6 & $\operatorname{Ir}(\text { ppy })_{3}$ & $\mathrm{Et}_{3} \mathrm{~N}$ & - & $\mathrm{MeCN}$ & 25 & $1: 1.5$ \\
\hline 7 & $\mathrm{Ru}(\mathrm{bpy})_{3} \mathrm{Cl}_{2} \cdot 6 \mathrm{H}_{2} \mathrm{O}$ & $\mathrm{Et}_{3} \mathrm{~N}$ & - & $\mathrm{MeCN}$ & n.r. & - \\
\hline 8 & EosinY & $\mathrm{Et}_{3} \mathrm{~N}$ & - & $\mathrm{MeCN}$ & n.r. & - \\
\hline 9 & Rhodamine B & $\mathrm{Et}_{3} \mathrm{~N}$ & - & $\mathrm{MeCN}$ & n.r. & - \\
\hline 10 & CBZ6 & $\mathrm{Et}_{3} \mathrm{~N}$ & $\mathrm{Sc}(\mathrm{OTf})_{3}(20 \mathrm{~mol} \%)$ & $\mathrm{MeCN}$ & 50 & $1: 1$ \\
\hline 11 & CBZ6 & $\mathrm{Et}_{3} \mathrm{~N}$ & ${ }^{n} \mathrm{Bu}_{4} \mathrm{NPF}_{6}(20 \mathrm{~mol} \%)$ & $\mathrm{MeCN}$ & 55 & $1: 1.2$ \\
\hline 12 & CBZ6 & $\mathrm{Et}_{3} \mathrm{~N}$ & - & DMF & 45 & $1: 1.2$ \\
\hline
\end{tabular}




\begin{tabular}{|c|c|c|c|c|c|c|}
\hline 13 & CBZ6 & $\mathrm{Et}_{3} \mathrm{~N}$ & - & DCM & 10 & 1:1 \\
\hline 14 & CBZ6 & $\mathrm{Et}_{3} \mathrm{~N}$ & - & THF & n.r. & - \\
\hline 15 & CBZ6 & $\mathrm{Et}_{3} \mathrm{~N}$ & - & $\mathrm{MeOH}$ & n.r. & - \\
\hline 16 & CBZ6 & $\mathrm{Et}_{3} \mathrm{~N}$ & - & DMSO & 70 & $1: 1$ \\
\hline 17 & CBZ6 & $\mathrm{Et}_{3} \mathrm{~N}$ & - & $\mathrm{PhCN}$ & 65 & $1: 1.2$ \\
\hline 18 & CBZ6 & $\mathrm{Et}_{3} \mathrm{~N}$ & - & $\mathrm{PhCl}$ & n.r. & - \\
\hline 19 & CBZ6 & - & - & $\mathrm{Et}_{3} \mathrm{~N}$ & 10 & 1:1 \\
\hline $20^{c}$ & CBZ6 & $\mathrm{Et}_{3} \mathrm{~N}$ & & $\mathrm{MeCN}$ & 55 & $1: 1.2$ \\
\hline $21^{d j}$ & CBZ6 & $\mathrm{Et}_{3} \mathrm{~N}$ & & $\mathrm{MeCN}$ & 54 & $1: 1.1$ \\
\hline $22^{e j}$ & CBZ6 & $\mathrm{Et}_{3} \mathrm{~N}$ & & $\mathrm{MeCN}$ & 70 & 1:1.1 \\
\hline $23^{f}$ & CBZ6 & $\mathrm{Et}_{3} \mathrm{~N}$ & - & $\mathrm{MeCN}$ & n.r. & \\
\hline $24^{g}$ & CBZ6 & $\mathrm{Et}_{3} \mathrm{~N}$ & - & $\mathrm{MeCN}$ & n.r. & \\
\hline $25^{h}$ & CBZ6 & $\mathrm{Et}_{3} \mathrm{~N}$ & - & $\mathrm{MeCN}$ & n.r. & \\
\hline $26^{i}$ & CBZ6 & $\mathrm{Et}_{3} \mathrm{~N}$ & - & $\mathrm{MeCN}$ & n.r. & \\
\hline $27^{j}$ & CBZ6 & $\mathrm{Et}_{3} \mathrm{~N}$ & - & $\mathrm{MeCN}$ & $72\left(74^{k}\right)$ & $1: 1.1$ \\
\hline
\end{tabular}

${ }^{a}$ Conditions: 1a $(0.2 \mathrm{mmol})$, solvent $(2 \mathrm{~mL}) .{ }^{b} \mathrm{H}$ NMR yield was reported using nitromethane and tert-Butyl methyl ether as an internal standard. n.r. $=$ no reaction. ${ }^{c} 18 \mathrm{~W}$ LEDs $(407 \mathrm{~nm}) .{ }^{d} 3 \mathrm{~W}$ LEDs $(407 \mathrm{~nm}) .{ }^{e} 6 \mathrm{~W}$ LEDs $(407 \mathrm{~nm}) .{ }^{f}$ Without light. ${ }^{g}$ Reaction under $\mathrm{O}_{2} .{ }^{h}$ Without Et 3 N. ${ }^{i}$ Without CBZ6. ${ }^{j} 0.25 \mathrm{M}(0.5 \mathrm{mmol} \mathrm{1a)}){ }^{k}$ Isolated yield.

\subsection{General procedure}

CBZ6 (1 mol \%, $2.2 \mathrm{mg}$ ) was added in an oven-dried $25 \mathrm{~mL}$ Schlenk tube. The reaction tube was placed under vacuum and backfilled with argon three times. Then aldehydes or ketones $(0.5 \mathrm{mmol}$, 1 equiv), $\mathrm{Et}_{3} \mathrm{~N}(0.5 \mathrm{mmol}, 69 \mu \mathrm{L})$ and dry $\mathrm{MeCN}(2 \mathrm{~mL})$ were added sequentially in the Schlenk tube via syringe under argon atmosphere. Then the tube was placed $5 \mathrm{~cm}$ away from LEDs $(9 \mathrm{~W}=$ $3 \mathrm{~W} \times 3 ; 18 \mathrm{~W}=3 \mathrm{~W} \times 6$ ), and stirred vigorously under the irradiation of light. Upon consumption of aldehydes or ketones (determined by TLC), the tube was removed from the light source. The crude product residue was purified by column chromatography on silica gel (PE/EA 20/1 to 3/1) to afford the purified product.<smiles>OC(c1ccccc1)C(O)c1ccccc1</smiles>

\section{1,2-diphenylethane-1,2-diol (2a) ${ }^{1}$}

This compound was prepared according to the general procedure under $9 \mathrm{~W}$ LEDs. Purified on silica gel chromatography (PE/EA 10/1 to 3/1) to give the product, $2.5 \mathrm{~h}$, white solid (39.5 mg, 74\%). meso/dl 1:1.1; ${ }^{1} \mathrm{H}$ NMR of (meso)- and $(\boldsymbol{d l})-\mathbf{2 a}\left(500 \mathrm{MHz}, \mathrm{CDCl}_{3}\right) \delta 7.34-7.29(\mathrm{~m}, 1.1 \times 6 \mathrm{H}, d l)$, 7.27-7.21 (m, 10H, meso), 7.14-7.12 (m, $1.1 \times 4 \mathrm{H}, d l), 4.84(\mathrm{~s}, 2 \mathrm{H}$, meso $), 4.72(\mathrm{~s}, 1.1 \times 2 \mathrm{H}, d l)$, $2.84(\mathrm{br} \mathrm{s}, 1.1 \times 2 \mathrm{H}, d l), 2.20$ (br s, $2 \mathrm{H}$, meso). ${ }^{13} \mathrm{C}$ NMR of (meso)- and (dl)-2a $\left(125 \mathrm{MHz}, \mathrm{CDCl}_{3}\right)$ $\delta 134.0,139.9,128.4,128.3(2 \mathrm{C}), 128.1,127.2,127.1,79.3,78.3$. 
<smiles>OC(c1ccc2ccccc2c1)C(O)c1ccc2ccccc2c1</smiles>

\section{1,2-di(naphthalen-2-yl)ethane-1,2-diol (2b) ${ }^{2}$}

This compound was prepared according to the general procedure under $9 \mathrm{~W}$ LEDs. Purified on silica gel chromatography (PE/EA 10/1 to 3/1) to give the product, $3.5 \mathrm{~h}$, yellow solid (68.9 mg, 88\%). meso/dl 1.1:1; ${ }^{1} \mathrm{H}$ NMR of (meso)- and (dl)-2b (500 MHz, $d^{6}$-DMSO) $\delta 7.86-7.81(\mathrm{~m}, 10.6 \mathrm{H})$, 7.75-7.69 (m, 7H), $7.51(\mathrm{~d}, J=8.0 \mathrm{~Hz}, 2 \mathrm{H}), 7.46-7.42(\mathrm{~m}, 8 \mathrm{H}), 7.33(\mathrm{~m}, J=7.9 \mathrm{~Hz}, 2 \mathrm{H}), 5.58(\mathrm{~s}$, $1.1 \times 2 \mathrm{H}$, meso), $5.45(\mathrm{~s}, 2 \mathrm{H}, d l), 4.92(\mathrm{~s}, 1.1 \times 2 \mathrm{H}$, meso $), 4.85(\mathrm{~s}, 2 \mathrm{H}, d l) .{ }^{13} \mathrm{C}$ NMR of $($ meso)and $(\boldsymbol{d l})-2 \mathbf{b}\left(125 \mathrm{MHz}, d^{6}\right.$-DMSO) $\delta 141.2,140.2,132.6,132.5,132.4,132.2,127.7,127.7,127.5$, $127.4,126.7,126.7,126.1,125.9,125.8,125.8,125.7,125.5,77.5,77.2$.<smiles>OC(c1ccc(-c2ccccc2)cc1)C(O)c1ccc(-c2ccccc2)cc1</smiles>

\section{1,2-di([1,1'-biphenyl]-4-yl)ethane-1,2-diol (2c) ${ }^{1}$}

This compound was prepared according to the general procedure under $9 \mathrm{~W}$ LEDs. Purified on silica gel chromatography (PE/EA 10/1 to 3/1) to give the product, $2.5 \mathrm{~h}$, yellow solid (85.6 mg, 93\%). meso/dl 1:1; ${ }^{1} \mathrm{H}$ NMR of (meso)- and (dl)-2c (400 MHz, $d^{6}$-DMSO) $\delta 7.66(\mathrm{~d}, J=7.4 \mathrm{~Hz}, 4 \mathrm{H})$, $7.63(\mathrm{~d}, J=7.3 \mathrm{~Hz}, 4 \mathrm{H}), 7.58(\mathrm{~d}, J=8.2 \mathrm{~Hz}, 4 \mathrm{H}), 7.52(\mathrm{~d}, J=8.2 \mathrm{~Hz}, 4 \mathrm{H}), 7.47-7.39(\mathrm{~m}, 12 \mathrm{H})$, 7.36-7.31 (m, 4H), 7.27 (d, J=8.2 Hz, 4H), 5.43 (s, 2H, meso), 5.32 (s, 2H, $d l), 4.71$ (s, 2H, meso), $4.64(\mathrm{~s}, 2 \mathrm{H}, d l) .{ }^{13} \mathbf{C}$ NMR of (meso)- and $(d l)-2 \mathrm{c}\left(100 \mathrm{MHz}, d^{6}\right.$-DMSO) $\delta 142.9,141.8,140.2$, 140.0, 138.5, 138.4, 128.9, 128.9, 128.0, 127.8, 127.2(2C), 126.5, 126.5, 125.7, 125.6, 77.1, 76.8.<smiles>Cc1ccc(C(O)C(O)c2ccc(C)cc2)cc1</smiles>

\section{1,2-di-p-tolylethane-1,2-diol (2d) ${ }^{3}$}

This compound was prepared according to the general procedure under $9 \mathrm{~W}$ LEDs. Purified on silica gel chromatography (PE/EA 10/1 to 3/1) to give the product, $2.5 \mathrm{~h}$, white solid $(59.4 \mathrm{mg}, 98 \%$ ). meso/dl 1:1.2; ${ }^{1} \mathrm{H}$ NMR of (meso)-2d $\left(400 \mathrm{MHz}, \mathrm{CDCl}_{3}\right) \delta 7.18(\mathrm{~d}, J=8.0 \mathrm{~Hz}, 4 \mathrm{H}), 7.14(\mathrm{~d}, J=$ $8.0 \mathrm{~Hz}, 4 \mathrm{H}), 4.73$ (s, 2H), 2.35 (s, 6H), 2.14 (s, 2H). ${ }^{13} \mathbf{C}$ NMR of (meso)-2d $\left(100 \mathrm{MHz}, \mathrm{CDCl}_{3}\right) \delta$ 138.0, 137.1, 129.1, 127.2, 78.2, 21.3. ${ }^{1} \mathbf{H}$ NMR of $(\boldsymbol{d l})-\mathbf{2 d}\left(400 \mathrm{MHz}, \mathrm{CDCl}_{3}\right) \delta 7.06-7.01(\mathrm{~m}, 8 \mathrm{H})$, $4.66(\mathrm{~s}, 2 \mathrm{H}), 2.86(\mathrm{~s}, 2 \mathrm{H}), 2.30(\mathrm{~s}, 6 \mathrm{H}) \cdot{ }^{13} \mathbf{C}$ NMR of $(\boldsymbol{d l})-\mathbf{2 d}\left(100 \mathrm{MHz}, \mathrm{CDCl}_{3}\right) \delta$ 137.6, 137.1, $128.9,127.0,78.9,21.3$.<smiles>Cc1cccc(C(O)C(O)c2cccc(C)c2)c1</smiles>

1,2-di-m-tolylethane-1,2-diol (2e $)^{4}$ 
This compound was prepared according to the general procedure under $9 \mathrm{~W}$ LEDs. Purified on silica gel chromatography (PE/EA 10/1 to 3/1) to give the product, $2.5 \mathrm{~h}$, yellow solid (44.4 mg, 73\%). meso/dl 1:1.3; ${ }^{1} \mathrm{H}$ NMR of (meso)-2e (400 MHz, $\left.\mathrm{CDCl}_{3}\right) \delta$ 7.25-7.21 (m, 2H), 7.13 (s, 4H), 7.117.09 (m, 2H), 4.71 (s, 2H), 2.35 (s, 6H), 2.15 (s, 2H). ${ }^{13}$ C NMR of (meso)-2e (100 MHz, CDCl $) \delta$ 140.1, 138.1, 129.1, 128.3, 127.9, 124.4, 78.3, 21.6. ${ }^{1} \mathbf{H}$ NMR of $(\boldsymbol{d l})-2 \mathbf{e}\left(400 \mathrm{MHz}, \mathrm{CDCl}_{3}\right) \delta$ 7.15$7.11(\mathrm{~m}, 2 \mathrm{H}), 7.05(\mathrm{~d}, J=7.6 \mathrm{~Hz}, 2 \mathrm{H}), 7.01(\mathrm{~s}, 2 \mathrm{H}), 6.92(\mathrm{~d}, J=7.5 \mathrm{~Hz}, 2 \mathrm{H}), 4.67(\mathrm{~s}, 2 \mathrm{H}), 2.86(\mathrm{~s}$, $2 \mathrm{H}), 2.29(\mathrm{~s}, 6 \mathrm{H}) .{ }^{13} \mathbf{C} \mathbf{~ N M R ~ o f ~}(\boldsymbol{d l})-2 \mathrm{e}\left(100 \mathrm{MHz}, \mathrm{CDCl}_{3}\right) \delta 140.0,137.9,128.7,128.1,127.6,124.1$, 78.9, 21.5.<smiles>Cc1ccccc1C(O)C(O)c1ccccc1C</smiles>

1,2-di-o-tolylethane-1,2-diol (2f) ${ }^{1}$

This compound was prepared according to the general procedure under $9 \mathrm{~W}$ LEDs. Purified on silica gel chromatography (PE/EA 10/1 to 3/1) to give the product, $2.5 \mathrm{~h}$, yellow oil $(51.2 \mathrm{mg}, 85 \%)$. meso/dl 1:1.5; ${ }^{1} \mathbf{H}$ NMR of (meso)- and (dl)-2f $\left(400 \mathrm{MHz}, \mathrm{CDCl}_{3}\right) \delta 7.60(\mathrm{~d}, J=7.7 \mathrm{~Hz}, 1.5 \times 2 \mathrm{H}$, $d l), 7.32-7.30(\mathrm{~m}, 2 \mathrm{H}$, meso $), 7.22-7.16(\mathrm{~m}, 7 \mathrm{H}, 1.5 \times 2 \mathrm{H} d l$ and $4 \mathrm{H}$ meso), $7.12(\mathrm{td}, J=7.5 \mathrm{~Hz}, 1.4$ $\mathrm{Hz}, 1.5 \times 2 \mathrm{H}, d l), 7.09-7.06(\mathrm{~m}, 2 \mathrm{H}$, meso $), 6.92(\mathrm{~d}, J=7.5 \mathrm{~Hz}, 1.5 \times 2 \mathrm{H}, d l), 5.17(\mathrm{~s}, 2 \mathrm{H}$, meso $)$, $4.94(\mathrm{~s}, 1.5 \times 2 \mathrm{H}, d l), 3.19(\mathrm{br} \mathrm{s}, 1.5 \times 2 \mathrm{H}, d l), 2.44(\mathrm{br} \mathrm{s}, 2 \mathrm{H}$, meso), 2.15 (s, $6 \mathrm{H}$, meso), $1.65(\mathrm{~s}, 1.5$ $\times 6 \mathrm{H}, d l) .{ }^{13} \mathbf{C} \mathbf{~ N M R ~ o f ~ ( m e s o ) - ~ a n d ~}(d l)-2 \mathrm{f}\left(100 \mathrm{MHz}, \mathrm{CDCl}_{3}\right) \delta 138.2,138.1,136.2,136.0,130.3$, $130.1,127.8,127.8,127.3,126.8,126.1,126.0,74.7,73.4,19.2,18.8$.<smiles>OC(c1ccc(F)cc1)C(O)c1ccc(F)cc1</smiles>

\section{1,2-bis(4-fluorophenyl)ethane-1,2-diol (2g) ${ }^{1}$}

This compound was prepared according to the general procedure under $9 \mathrm{~W}$ LEDs. Purified on silica gel chromatography (PE/EA 10/1 to 3/1) to give the product, $2.5 \mathrm{~h}$, white solid (43.0 mg, 69\%). meso/dl 1.2:1; ${ }^{1} \mathrm{H}$ NMR of (meso)- and (dl)-2g (500 MHz, $d^{6}$-DMSO) $\delta$ 7.24-7.21 (m, 4H, $\left.d l\right)$, 7.09-7.04 (m, 8.8H, $4 \mathrm{H} d l$ and $1.2 \times 4 \mathrm{H} \mathrm{meso),} \mathrm{7.00-6.97} \mathrm{(m,} 1.2 \times 4 \mathrm{H}$, meso), $5.46(\mathrm{~s}, 1.2 \times 2 \mathrm{H}$, meso), $5.32(\mathrm{~s}, 2 \mathrm{H}, d l), 4.60(\mathrm{~s}, 1.2 \times 2 \mathrm{H}$, meso $), 4.56(\mathrm{~s}, 2 \mathrm{H}, d l) \cdot{ }^{13} \mathrm{C}$ NMR of (meso)- and $(d l)-2 \mathrm{~g}$ $\left(125 \mathrm{MHz}, d^{6}\right.$-DMSO) $\delta 161.2\left(\mathrm{C}-\mathrm{F}, 1 J_{\mathrm{C}-\mathrm{F}}=240.3 \mathrm{~Hz}\right), 161.1\left(\mathrm{C}-\mathrm{F}, 1 J_{\mathrm{C}-\mathrm{F}}=240.6 \mathrm{~Hz}\right), 139.2(\mathrm{C}-\mathrm{F}$, $\left.4 J_{\mathrm{C}-\mathrm{F}}=2.5 \mathrm{~Hz}\right), 138.3\left(\mathrm{C}-\mathrm{F}, 4 J_{\mathrm{C}-\mathrm{F}}=2.6 \mathrm{~Hz}\right), 129.1\left(\mathrm{C}-\mathrm{F}, 3 J_{\mathrm{C}-\mathrm{F}}=7.8 \mathrm{~Hz}\right), 128.9\left(\mathrm{C}-\mathrm{F}, 3 J_{\mathrm{C}-\mathrm{F}}=7.8\right.$ $\mathrm{Hz}), 114.0\left(\mathrm{C}-\mathrm{F}, 2 J_{\mathrm{C}-\mathrm{F}}=20.9 \mathrm{~Hz}\right), 113.9\left(\mathrm{C}-\mathrm{F}, 2 J_{\mathrm{C}-\mathrm{F}}=20.9 \mathrm{~Hz}\right), 76.6,76.2 .{ }^{19} \mathbf{F}$ NMR of (meso)and $(\boldsymbol{d l})$-2g $\left(471 \mathrm{MHz}, d^{6}\right.$-DMSO, decoupled) $\delta-116.3,-116.4$.<smiles>OC(c1ccc(Cl)cc1)C(O)c1ccc(Cl)cc1</smiles>

\section{1,2-bis(4-chlorophenyl)ethane-1,2-diol (2h) ${ }^{1}$}

This compound was prepared according to the general procedure under $9 \mathrm{~W}$ LEDs. Purified on silica gel chromatography (PE/EA 10/1 to 3/1) to give the product, $2.5 \mathrm{~h}$, white solid (48.9 mg, 69\%). 
meso/dl 1:1.1; ${ }^{1} \mathrm{H}$ NMR of (meso)- and (dl)-2h $\left(400 \mathrm{MHz}, \mathrm{CDCl}_{3}\right) \delta 7.25(\mathrm{~d}, J=8.2 \mathrm{~Hz}, 4 \mathrm{H}$, meso), $7.20(\mathrm{~d}, J=8.2 \mathrm{~Hz}, 1.1 \times 4 \mathrm{H}, d l), 7.09(\mathrm{~d}, J=8.2 \mathrm{~Hz}, 4 \mathrm{H}, m e s o), 7.00(\mathrm{~d}, J=8.2 \mathrm{~Hz}, 1.1 \times 4 \mathrm{H}, d l)$, $4.81(\mathrm{~s}, 2 \mathrm{H}$, meso $), 4.59(\mathrm{~s}, 1.1 \times 2 \mathrm{H}, d l), 2.99(\mathrm{~s}, 1.1 \times 2 \mathrm{H}, d l), 2.42\left(\mathrm{~s}, 2 \mathrm{H}\right.$, meso) ${ }^{13} \mathbf{C}$ NMR of (meso)- and (dl)-2h $\left(100 \mathrm{MHz}, \mathrm{CDCl}_{3}\right) \delta 138.0,137.9,134.0,128.5,128.5,128.5,78.7,77.3$.<smiles>COc1ccc(C(O)C(O)c2ccc(OC)cc2)cc1</smiles>

\section{1,2-bis(4-methoxyphenyl)ethane-1,2-diol (2i) ${ }^{1}$}

This compound was prepared according to the general procedure under $9 \mathrm{~W}$ LEDs. Purified on silica gel chromatography (PE/EA 10/1 to 3/1) to give the product, $2.5 \mathrm{~h}$, yellow oil (47.0 mg, 69\%). meso/dl 1:1.7; ${ }^{1} \mathrm{H}$ NMR of (meso)- and (dl)-2i $\left(500 \mathrm{MHz}, \mathrm{CDCl}_{3}\right) \delta 7.20(\mathrm{~d}, J=8.7 \mathrm{~Hz}, 4 \mathrm{H}$, meso), $7.03(\mathrm{~d}, J=8.8 \mathrm{~Hz}, 1.7 \times 4 \mathrm{H}, d l), 6.86(\mathrm{~d}, J=8.8 \mathrm{~Hz}, 4 \mathrm{H}, m e s o), 6.76(\mathrm{~d}, J=8.8 \mathrm{~Hz}, 1.7 \times 4 \mathrm{H}, d l)$, $4.73(\mathrm{~s}, 2 \mathrm{H}$, meso), $4.62(\mathrm{~s}, 1.7 \times 2 \mathrm{H}, d l), 3.80(\mathrm{~s}, 6 \mathrm{H}$, meso $), 3.76(\mathrm{~s}, 1.7 \times 6 \mathrm{H}, d l), 2.83(\mathrm{~s}, 1.7 \times$ $2 \mathrm{H}, d l), 2.12\left(\mathrm{~s}, 2 \mathrm{H}\right.$, meso). ${ }^{13} \mathbf{C}$ NMR of (meso)- and $(\boldsymbol{d l})-\mathbf{2 i}\left(125 \mathrm{MHz}, \mathrm{CDCl}_{3}\right) \delta 159.6,159.3$, $132.2,132.2,128.5,128.3,113.8,113.6,78.9,77.9,55.4,55.3$.<smiles>OC(c1ccc(C(F)(F)F)cc1)C(O)c1ccc(C(F)(F)F)cc1</smiles>

\section{1,2-bis(4-(trifluoromethyl)phenyl)ethane-1,2-diol (2j) $)^{2}$}

This compound was prepared according to the general procedure using $\mathrm{Et}_{3} \mathrm{~N}(1.0 \mathrm{mmol}, 138 \mu \mathrm{L})$ under 18 W LEDs. Purified on silica gel chromatography (PE/EA 10/1 to 3/1) to give the product, $12 \mathrm{~h}$, white solid (57.0 mg, 65\%). meso/dl 1:1.1; ${ }^{1} \mathbf{H}$ NMR of (meso)- and (dl)-2j (400 MHz, $\left.\mathrm{CDCl}_{3}\right)$ $\delta 7.55-7.50(\mathrm{~m}, 8.5 \mathrm{H}, 1.1 \times 4 \mathrm{H} d l$ and $4 \mathrm{H} m e s o), 7.28(\mathrm{~d}, J=8.2 \mathrm{~Hz}, 4 \mathrm{H}, m e s o), 7.22(\mathrm{~d}, J=8.0 \mathrm{~Hz}$, $1.1 \times 4 \mathrm{H}, d l), 4.95(\mathrm{~s}, 2 \mathrm{H}$, meso $), 4.74(\mathrm{~s}, 1.1 \times 2 \mathrm{H}, d l), 3.02(\mathrm{br} \mathrm{s}, 1.1 \times 2 \mathrm{H}, d l), 2.50(\mathrm{br} \mathrm{s}, 2 \mathrm{H}$, meso). ${ }^{13} \mathbf{C}$ NMR of (meso)- and (dl)-2j (100 MHz, $\left.\mathrm{CDCl}_{3}\right) \delta 143.4,143.3,130.6\left(\mathrm{C}-\mathrm{F}, 2 J_{\mathrm{C}-\mathrm{F}}=32.2\right.$ $\mathrm{Hz}), 130.5\left(\mathrm{C}-\mathrm{F}, 2 J_{\mathrm{C}-\mathrm{F}}=32.1 \mathrm{~Hz}\right), 127.5,127.4,125.4\left(\mathrm{C}-\mathrm{F}, 3 J_{\mathrm{C}-\mathrm{F}}=3.6 \mathrm{~Hz}\right), 125.2\left(\mathrm{C}-\mathrm{F}, 3 J_{\mathrm{C}-\mathrm{F}}=3.6\right.$ $\mathrm{Hz}), 124.1\left(\mathrm{C}-\mathrm{F}, 1 J_{\mathrm{C}-\mathrm{F}}=270.3 \mathrm{~Hz}\right), 124.1\left(\mathrm{C}-\mathrm{F}, 1 J_{\mathrm{C}-\mathrm{F}}=270.3 \mathrm{~Hz}\right), 78.5,77.3 .{ }^{19} \mathbf{F}$ NMR of $(\boldsymbol{m e s o})-$ and $(d l)-2 \mathbf{j}\left(376 \mathrm{MHz}, \mathrm{CDCl}_{3}\right.$, decoupled) $\delta-62.6,-62.6$.<smiles>COC(=O)c1ccc(C(O)C(O)c2ccc(C(=O)OC)cc2)cc1</smiles>

\section{dimethyl 4,4'-(1,2-dihydroxyethane-1,2-diyl)dibenzoate (2k) ${ }^{1}$}

This compound was prepared according to the general procedure using $\mathrm{Et}_{3} \mathrm{~N}(1.0 \mathrm{mmol}, 138 \mu \mathrm{L})$ under 18 W LEDs. Purified on silica gel chromatography (PE/EA 10/1 to 3/1) to give the product, $12 \mathrm{~h}$, yellow solid (63.2 mg, 77\%). meso/dl 1.3:1; ${ }^{1} \mathrm{H}$ NMR of (meso)- and (dl)-2k (500 MHz, $d^{6}$ DMSO) $\delta 7.85(\mathrm{~d}, J=7.8 \mathrm{~Hz}, 4 \mathrm{H}, d l), 7.77(\mathrm{~d}, J=7.8 \mathrm{~Hz}, 1.3 \times 4 \mathrm{H}, m e s o), 7.36(\mathrm{~d}, J=7.8 \mathrm{~Hz}, 4 \mathrm{H}$, 
$d l), 7.24(\mathrm{~d}, J=7.8 \mathrm{~Hz}, 1.3 \times 4 \mathrm{H}$, meso), $5.67(\mathrm{~s}, 1.3 \times 2 \mathrm{H}$, meso), $5.56(\mathrm{~s}, 2 \mathrm{H}, d l), 4.76(\mathrm{~s}, 1.3 \times$ $2 \mathrm{H}$, meso), $4.68(\mathrm{~s}, 2 \mathrm{H}, d l), 3.83(\mathrm{~s}, 6 \mathrm{H}, d l), 3.81\left(\mathrm{~s}, 1.3 \times 6 \mathrm{H}\right.$, meso). ${ }^{13} \mathrm{C} \mathrm{NMR} \mathrm{of} \mathrm{(meso)-} \mathrm{and} \mathrm{(dl)-}$ 2k (125 MHz, $d^{6}$-DMSO) $\delta 166.3,166.3,148.5,147.7,128.3,128.2,128.1,128.0,127.7,127.4$, $76.7,76.5,52.0(2 \mathrm{C})$.<smiles>N#Cc1ccc(C(O)C(O)c2ccc(C#N)cc2)cc1</smiles>

\section{4,4'-(1,2-dihydroxyethane-1,2-diyl)dibenzonitrile (2l) ${ }^{2}$}

This compound was prepared according to the general procedure using $\mathrm{Et}_{3} \mathrm{~N}(1.0 \mathrm{mmol}, 138 \mu \mathrm{L})$ under 18 W LEDs. Purified on silica gel chromatography (PE/EA 10/1 to 3/1) to give the product, $12 \mathrm{~h}$, orange solid (51.8 mg, 78\%). meso/dl 1.1:1; ${ }^{1} \mathbf{H}$ NMR of (meso)- and (dl)-2l (500 MHz, $d^{6}$ DMSO) $\delta 7.73(\mathrm{~d}, J=7.8 \mathrm{~Hz}, 4 \mathrm{H}, d l), 7.68(\mathrm{~d}, J=7.8 \mathrm{~Hz}, 1.1 \times 4 \mathrm{H}, m e s o), 7.42(\mathrm{~d}, J=7.8 \mathrm{~Hz}, 4 \mathrm{H}$, $d l), 7.33(\mathrm{~d}, J=7.8 \mathrm{~Hz}, 1.1 \times 4 \mathrm{H}$, meso), $5.75(\mathrm{~s}, 1.1 \times 2 \mathrm{H}$, meso $), 5.69(\mathrm{~s}, 2 \mathrm{H}, d l), 4.82(\mathrm{~s}, 1.1 \times$ $2 \mathrm{H}$, meso), 4.67 (s, 2H, $d l) .{ }^{13} \mathrm{C}$ NMR of (meso)- and (dl)-2l (125 MHz, $d^{6}$-DMSO) $\delta 148.5,147.9$, $131.4,131.3,128.3,128.0,119.1,109.6,109.5,76.1,75.9$.<smiles>OC(c1cccs1)C(O)c1ccc(-c2cccs2)s1</smiles>

\section{1,2-di([2,2'-bithiophen]-5-yl)ethane-1,2-diol (2m) ${ }^{2}$}

This compound was prepared according to the general procedure under $9 \mathrm{~W}$ LEDs. Purified on silica gel chromatography (PE/EA 10/1 to 3/1) to give the product, $12 \mathrm{~h}$, orange solid (61.4 mg, 63\%). meso/dl 1.15:1; ${ }^{1} \mathbf{H}$ NMR of (meso)- and (dl)-2m (400 MHz, $d^{6}$-DMSO) $\delta$ 7.46-7.44 (m, 4.3H, 1.15 $\times 2 \mathrm{H} \mathrm{meso}$ and $2 \mathrm{H} d l), 7.22(\mathrm{~d}, J=3.0 \mathrm{~Hz}, 2 \mathrm{H}, d l), 7.20(\mathrm{~d}, J=2.8 \mathrm{~Hz}, 1.15 \times 2 \mathrm{H}$, meso $), 7.10(\mathrm{~d}$, $J=3.6 \mathrm{~Hz}, 2 \mathrm{H}, d l), 7.07-7.04(\mathrm{~m}, 6.5 \mathrm{H}, 1.15 \times 3 \mathrm{H}$ meso and $3 \mathrm{H} d l), 6.91(\mathrm{~d}, J=3.6 \mathrm{~Hz}, 2 \mathrm{H}, d l)$, $6.77(\mathrm{~d}, J=3.6 \mathrm{~Hz}, 1.15 \times 2 \mathrm{H}$, meso $), 6.09-6.08(\mathrm{~m}, 4.3 \mathrm{H}, 1.15 \times 2 \mathrm{H}$ meso and $2 \mathrm{H} d l), 4.89(\mathrm{~d}, J=$ $2.4 \mathrm{~Hz}, 1.15 \times 2 \mathrm{H}$, meso $), 4.83(\mathrm{~d}, J=2.7 \mathrm{~Hz}, 2 \mathrm{H}, d l) .{ }^{13} \mathbf{C ~ N M R ~ o f ~ ( m e s o ) - ~ a n d ~}(d l)-2 \mathrm{~m}(100 \mathrm{MHz}$, $d^{6}$-DMSO) $\delta$ 146.0, 145.0, 137.1, 137.0, 135.3, 135.2, 128.3 (2C), 125.6, 125.5, 124.9 (2C), 123.4 (2C), 122.9 (2C), 73.3, 73.1.<smiles>CC1CCC(C(C)C)C(OC(=O)c2ccc(C(O)C(O)c3ccc(C(=O)OC4CC(C)CCC4C(C)C)cc3)cc2)C1</smiles>

bis(2-isopropyl-5-methylcyclohexyl) 4,4'-(1,2-dihydroxyethane-1,2-diyl)dibenzoate (2n)

This compound was prepared according to the general procedure using $\mathrm{Et}_{3} \mathrm{~N}(1.0 \mathrm{mmol}, 138 \mu \mathrm{L})$ under 18 W LEDs. Purified on silica gel chromatography (PE/EA 10/1 to 3/1) to give the product, $12 \mathrm{~h}$, yellow oil (105.7 mg, 73\%). meso/dl 1:1.23; ${ }^{1} \mathbf{H}$ NMR of (meso)- and (dl)-2n (400 MHz, $\left.\mathrm{CDCl}_{3}\right) \delta 7.94(\mathrm{~d}, J=8.0 \mathrm{~Hz}, 4 \mathrm{H}$, meso $), 7.89(\mathrm{~d}, J=8.0 \mathrm{~Hz}, 1.23 \times 4 \mathrm{H}, d l), 7.25(\mathrm{~d}, J=8.3 \mathrm{~Hz}$, $4 \mathrm{H}$, meso $), 7.18(\mathrm{t}, J=7.5 \mathrm{~Hz}, 1.23 \times 4 \mathrm{H}, d l), 4.97-4.87(\mathrm{~m}, 6.5 \mathrm{H}, 1.23 \times 2 \mathrm{H} d l$ and $4 \mathrm{H}$ meso $), 4.75$ $(\mathrm{d}, J=8.4 \mathrm{~Hz}, 1.23 \times 2 \mathrm{H}, d l), 3.09-3.06(\mathrm{~m}, 1.23 \times 2 \mathrm{H}, d l), 2.52(\mathrm{~s}, 2 \mathrm{H}, m e s o), 2.11(\mathrm{~d}, J=11.3 \mathrm{~Hz}$, 
$4.5 \mathrm{H}, 1.23 \times 2 \mathrm{H} d l$ and $2 \mathrm{H}$ meso $), 1.95-1.91(\mathrm{~m}, 4.46 \mathrm{H}, 1.23 \times 2 \mathrm{H} d l$ and $2 \mathrm{H}$ meso), $1.72(\mathrm{~d}, J=$ $11.3 \mathrm{~Hz}, 9 \mathrm{H}, 1.23 \times 4 \mathrm{H} d l$ and $4 \mathrm{H} \mathrm{meso}), 1.56-1.51(\mathrm{~m}, 9 \mathrm{H}, 1.23 \times 4 \mathrm{H} d l$ and $4 \mathrm{H}$ meso $), 1.17-1.05$ (m, 9H, $1.23 \times 4 \mathrm{H} d l$ and $4 \mathrm{H}$ meso), 0.96-0.88 (m, 31H, $1.23 \times 14 \mathrm{H} d l$ and $14 \mathrm{H}$ meso), 0.79-0.77 $\left(\mathrm{m}, 13.5 \mathrm{H}, 1.23 \times 6 \mathrm{H} \mathrm{dl}\right.$ and $6 \mathrm{H}$ meso). ${ }^{13} \mathbf{C} \mathbf{~ N M R}$ of (meso)- and $(d l)-2 \mathbf{n}\left(100 \mathrm{MHz}, \mathrm{CDCl}_{3}\right) \delta$ 166.0 (2C), 144.5 (2C), 130.5 (2C), 129.6, 129.5, 127.1, 127.1, 78.8, 75.1, 47.3 (2C), 41.0 (2C), 34.4 (2C), 31.5 (2C), 26.6 (2C), 23.7 (2C), 22.2 (2C), 20.9 (2C), 16.6 (2C). HRMS (ESI- TOF) $\mathrm{m} / \mathrm{z}:[\mathrm{M}+\mathrm{Na}]^{+}$calcd for $\mathrm{C}_{36} \mathrm{H}_{50} \mathrm{NaO}_{6}{ }^{+} 601.3500$, found 601.3500 .<smiles>OC(c1ccccc1)(c1ccccc1)C(O)(c1ccccc1)c1ccccc1</smiles>

\section{1,1,2,2-tetraphenylethane-1,2-diol (2o) ${ }^{1}$}

This compound was prepared according to the general procedure under $9 \mathrm{~W}$ LEDs. Purified on silica gel chromatography (PE/EA 20/1 to 10/1) to give the product, $2.5 \mathrm{~h}$, white solid (64.2 $\mathrm{mg}, 70 \%)$. ${ }^{1} \mathbf{H}$ NMR of $2 \mathbf{o}\left(400 \mathrm{MHz}, \mathrm{CDCl}_{3}\right) \delta$ 7.32-7.30 (m, 8H), 7.20-7.16 (m, 12H), 3.05 (s, 2H). ${ }^{13} \mathbf{C}$ NMR of $2 \mathbf{o}\left(100 \mathrm{MHz}, \mathrm{CDCl}_{3}\right) \delta 144.3,128.7,127.4,127.1,83.1$.<smiles>OC(c1ccccc1)(c1ccc(Cl)cc1)C(O)(c1ccccc1)c1ccc(Cl)cc1</smiles>

\section{1,2-bis(4-chlorophenyl)-1,2-diphenylethane-1,2-diol (2p) ${ }^{5}$}

This compound was prepared according to the general procedure under $9 \mathrm{~W}$ LEDs. Purified on silica gel chromatography (PE/EA 30/1 to 15/1) to give the product, $2.5 \mathrm{~h}$, white solid $(79.8 \mathrm{mg}, 73 \%)$. meso/dl 1:1.15; ${ }^{1} \mathbf{H}$ NMR of (meso)- and (dl)-2p $\left(400 \mathrm{MHz}, \mathrm{CDCl}_{3}\right) \delta 7.41(\mathrm{~d}, J=8.6 \mathrm{~Hz}, 1.15 \times$ $4 \mathrm{H}, d l), 7.26-7.23(\mathrm{~m}, 10 \mathrm{H}), 7.21-7.19(\mathrm{~m}, 11 \mathrm{H}), 7.16-7.13(\mathrm{~m}, 9 \mathrm{H}), 7.08(\mathrm{~d}, J=7.0 \mathrm{~Hz}, 1.15 \times 4 \mathrm{H}$, $d l), 2.97(\mathrm{~s}, 1.15 \times 2 \mathrm{H}, d l), 2.94\left(\mathrm{~s}, 2 \mathrm{H}\right.$, meso). ${ }^{13} \mathbf{C}$ NMR of (meso)- and $(d l)-2 p\left(100 \mathrm{MHz}, \mathrm{CDCl}_{3}\right)$ $\delta$ 143.6, 143.3, $142.8(2 \mathrm{C}), 133.1,132.9,130.3,130.2,128.5,128.4,127.7,127.7,127.6(2 \mathrm{C}), 127.5$ (2C), 82.9 (2C).<smiles>Cc1ccc(C(O)(c2ccccc2)C(O)(c2ccccc2)c2ccc(C)cc2)cc1</smiles>

\section{1,2-diphenyl-1,2-di-p-tolylethane-1,2-diol (2q) $)^{1}$}

This compound was prepared according to the general procedure under $9 \mathrm{~W}$ LEDs. Purified on silica gel chromatography (PE/EA 40/1 to 20/1) to give the product, $3.5 \mathrm{~h}$, white solid (75.5 mg, 77\%). meso/dl 1:1; ${ }^{1} \mathbf{H}$ NMR of (meso)- and (dl)-2q (400 MHz, $\left.\mathrm{CDCl}_{3}\right) \delta$ 7.37-7.32 (m, 8H), 7.20-7.14 (m, 20H), 7.02-6.98 (m, 8H), 3.03 (s, 4H, 2H dl and 2H meso), 2.31 (s, 6H, $d l$ ), 2.30 (s, 6H, meso). ${ }^{13} \mathbf{C}$ NMR of (meso)- and (dl)-2q $\left(100 \mathrm{MHz}, \mathrm{CDCl}_{3}\right) \delta 144.6,144.5,141.3,141.3,136.7,136.6$, $128.7,128.7,128.6,128.6,128.2,128.1,127.3,127.3,126.9,126.8,83.0$ (2C), 21.1 (2C). 


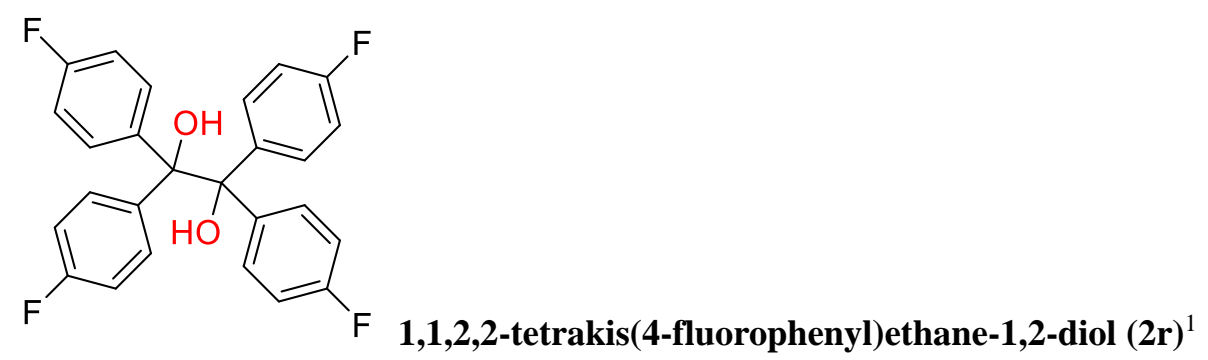

This compound was prepared according to the general procedure under $9 \mathrm{~W}$ LEDs. Purified on silica gel chromatography (PE/EA 40/1 to 20/1) to give the product, $3.5 \mathrm{~h}$, white solid $(84.7 \mathrm{mg}, 77 \%$ ). ${ }^{1} \mathbf{H}$ NMR of $2 \mathbf{r}\left(400 \mathrm{MHz}, \mathrm{CDCl}_{3}\right) \delta 7.26-7.23(\mathrm{~m}, 8 \mathrm{H}), 6.90-6.85(\mathrm{~m}, 8 \mathrm{H}), 2.86(\mathrm{~s}, 2 \mathrm{H}) .{ }^{13} \mathbf{C ~ N M R}$ of $2 \mathbf{r}\left(100 \mathrm{MHz}, \mathrm{CDCl}_{3}\right) \delta 161.9\left(\mathrm{C}-\mathrm{F}, 1 J_{\mathrm{C}-\mathrm{F}}=246.0 \mathrm{~Hz}\right), 139.8\left(\mathrm{C}-\mathrm{F}, 4 J_{\mathrm{C}-\mathrm{F}}=3.2 \mathrm{~Hz}\right), 130.4(\mathrm{C}-\mathrm{F}$, $\left.3 J_{\mathrm{C}-\mathrm{F}}=7.9 \mathrm{~Hz}\right), 114.4\left(\mathrm{C}-\mathrm{F}, 2 J_{\mathrm{C}-\mathrm{F}}=21.1 \mathrm{~Hz}\right), 82.7 .{ }^{19} \mathbf{F} \mathbf{N M R}$ of $2 \mathbf{r}\left(376 \mathrm{MHz}, \mathrm{CDCl}_{3}\right.$, decoupled $)$ $\delta-115.0$<smiles>OC(c1ccc(Cl)cc1)(c1ccc(Cl)cc1)C(O)(c1ccc(Cl)cc1)c1ccc(Cl)cc1</smiles>

\section{1,1,2,2-tetrakis(4-chlorophenyl)ethane-1,2-diol (2s) ${ }^{1}$}

This compound was prepared according to the general procedure under $9 \mathrm{~W}$ LEDs. Purified on silica gel chromatography (PE/EA 30/1 to $15 / 1$ ) to give the product, $4 \mathrm{~h}$, white solid $(81.4 \mathrm{mg}, 65 \%) .{ }^{1} \mathbf{H}$ NMR of $2 \mathrm{~s}\left(400 \mathrm{MHz}, \mathrm{CDCl}_{3}\right) \delta 7.21(\mathrm{~d}, J=7.0 \mathrm{~Hz}, 8 \mathrm{H}), 7.16(\mathrm{~d}, J=7.0 \mathrm{~Hz}, 8 \mathrm{H}), 2.85(\mathrm{~s}, 2 \mathrm{H})$. ${ }^{13}$ C NMR of $2 s\left(100 \mathrm{MHz}, \mathrm{CDCl}_{3}\right) \delta 142.1,133.6,130.0,127.9,82.6$.<smiles>Cc1ccc(C(O)(c2ccc(C)cc2)C(O)(c2ccc(C)cc2)c2ccc(C)cc2)cc1</smiles>

This compound was prepared according to the general procedure under $9 \mathrm{~W}$ LEDs. Purified on silica gel chromatography (PE/EA 40/1 to 20/1) to give the product, $8 \mathrm{~h}$, white solid $(69.8 \mathrm{mg}, 66 \%)$. ${ }^{1} \mathbf{H}$ NMR of $2 \mathrm{t}\left(400 \mathrm{MHz}, \mathrm{CDCl}_{3}\right) \delta 7.16(\mathrm{~d}, J=8.2 \mathrm{~Hz}, 8 \mathrm{H}), 6.98(\mathrm{~d}, J=8.1 \mathrm{~Hz}, 8 \mathrm{H}), 2.97(\mathrm{~s}, 2 \mathrm{H})$, $2.29(\mathrm{~s}, 12 \mathrm{H}) .{ }^{13} \mathrm{C}$ NMR of $2 \mathrm{t}\left(100 \mathrm{MHz}, \mathrm{CDCl}_{3}\right) \delta 141.6,136.4,128.6,128.1,83.0,21.1$.<smiles>CC(O)(c1ccccc1)C(C)(O)c1ccccc1</smiles>

\section{2,3-diphenylbutane-2,3-diol (2u) ${ }^{1}$}

This compound was prepared according to the general procedure under $18 \mathrm{~W}$ LEDs. Purified on silica gel chromatography (PE/EA 30/1 to 10/1) to give the product, $12 \mathrm{~h}$, white solid (42.2 mg, 
70\%). meso/dl 1:1.2; ${ }^{1} \mathrm{H}$ NMR of (meso)- and (dl)-2u (400 MHz, $\left.\mathrm{CDCl}_{3}\right) \delta$ 7.27-7.19 (m, 22H, 1.2 $\times 10 \mathrm{H} d l$ and $10 \mathrm{H}$ meso), $2.61(\mathrm{~d}, J=8.2 \mathrm{~Hz}, 1.2 \times 2 \mathrm{H}, d l), 2.31(\mathrm{~d}, J=7.1 \mathrm{~Hz}, 2 \mathrm{H}$, meso $), 1.59(\mathrm{~s}$, $6 \mathrm{H}$, meso), $1.51(\mathrm{~s}, 1.2 \times 6 \mathrm{H}, d l) .{ }^{13} \mathbf{C}$ NMR of (meso)- and $(d l)-2 \mathbf{u}\left(100 \mathrm{MHz}, \mathrm{CDCl}_{3}\right) \delta 143.9$, $143.5,127.5,127.4,127.3,127.2,127.0$ (2C), 79.0, 78.7, 25.2, 25.1.<smiles>CC(O)(c1ccc(C#N)cc1)C(C)(O)c1ccc(C#N)cc1</smiles>

\section{4,4'-(2,3-dihydroxybutane-2,3-diyl)dibenzonitrile $(2 \mathrm{v})^{7}$}

This compound was prepared according to the general procedure under $9 \mathrm{~W}$ LEDs. Purified on silica gel chromatography (PE/EA 10/1 to 3/1) to give the product, $8 \mathrm{~h}$, white solid $(59.5 \mathrm{mg}, 81 \%)$. meso/dl 1.7:1; ${ }^{1} \mathrm{H}$ NMR of (meso)- and (dl)-2v (500 MHz, $d^{6}$-DMSO) $\delta 7.74(\mathrm{~d}, J=8.5 \mathrm{~Hz}, 4 \mathrm{H}$, $d l), 7.68(\mathrm{~d}, J=8.4 \mathrm{~Hz}, 4 \mathrm{H}, d l), 7.51(\mathrm{~d}, J=8.6 \mathrm{~Hz}, 1.7 \times 4 \mathrm{H}, m e s o), 7.23(\mathrm{~d}, J=8.3 \mathrm{~Hz}, 1.7 \times 4 \mathrm{H}$, meso), $5.55\left(\mathrm{~s}, 1.7 \times 2 \mathrm{H}\right.$, meso), $5.38(\mathrm{~s}, 2 \mathrm{H}, d l), 1.61(\mathrm{~s}, 1.7 \times 6 \mathrm{H}$, meso $), 1.23(\mathrm{~s}, 6 \mathrm{H}, d l) .{ }^{13} \mathrm{C} \mathrm{NMR}$ of (meso)- and $(d l)-2 \mathbf{v}\left(125 \mathrm{MHz}, d^{6}\right.$-DMSO) $\delta 152.4,152.1,130.6,130.2,128.7,128.0,119.2$, $119.2,108.9,108.5,77.0,76.8,24.6,24.3$.<smiles>CC(O)(c1ccc(C(F)(F)F)cc1)C(C)(O)c1ccc(C(F)(F)F)cc1</smiles>

\section{2,3-bis(4-(trifluoromethyl)phenyl)butane-2,3-diol (2w) ${ }^{1}$}

This compound was prepared according to the general procedure under $9 \mathrm{~W}$ LEDs. Purified on silica gel chromatography (PE/EA 15/1 to 5/1) to give the product, $8 \mathrm{~h}$, yellow oil $(68.9 \mathrm{mg}, 73 \%)$. meso/dl $1: 1.15 ;{ }^{1} \mathrm{H}$ NMR of (meso)- and $(d l)-2 \mathbf{w}\left(400 \mathrm{MHz}, \mathrm{CDCl}_{3}\right) \delta 7.52-7.50(\mathrm{~m}, 8.6 \mathrm{H}, 1.15 \times 4 \mathrm{H} d l$ and 4H meso), $7.44(\mathrm{~d}, J=8.0 \mathrm{~Hz}, 4 \mathrm{H}, m e s o), 7.29(\mathrm{~d}, J=8.0 \mathrm{~Hz}, 1.15 \times 4 \mathrm{H}, d l), 2.62(\mathrm{br} \mathrm{s}, 1.15 \times 2 \mathrm{H}$, $d l), 2.23$ (s, 2H, meso), 1.57 (s, 6H, meso), 1.53 (s, $1.15 \times 6 \mathrm{H}, d l) .{ }^{13} \mathrm{C}$ NMR of (meso)- and (dl)$2 \mathbf{w}\left(100 \mathrm{MHz}, \mathrm{CDCl}_{3}\right) \delta 147.8,147.2,129.7\left(\mathrm{C}-\mathrm{F}, 2 J_{\mathrm{C}-\mathrm{F}}=32.2 \mathrm{~Hz}\right), 129.5\left(\mathrm{C}-\mathrm{F}, 2 J_{\mathrm{C}-\mathrm{F}}=32.3 \mathrm{~Hz}\right)$, 127.9, 127.6, $124.4\left(\mathrm{C}-\mathrm{F}, 3 J_{\mathrm{C}-\mathrm{F}}=3.6 \mathrm{~Hz}\right), 124.3\left(\mathrm{C}-\mathrm{F}, 3 J_{\mathrm{C}-\mathrm{F}}=3.6 \mathrm{~Hz}\right), 124.3\left(\mathrm{C}-\mathrm{F}, 1 J_{\mathrm{C}-\mathrm{F}}=271.0 \mathrm{~Hz}\right)$, $124.3\left(\mathrm{C}-\mathrm{F}, 1 J_{\mathrm{C}-\mathrm{F}}=270.2 \mathrm{~Hz}\right), 78.7,78.4,25.4,24.9 .{ }^{19} \mathbf{F}$ NMR of (meso)- and $(d l)-2 \mathbf{w}(376 \mathrm{MHz}$, $\mathrm{CDCl}_{3}$, decoupled) $\delta-62.4,-62.5$.<smiles>CC(O)(c1ccc2ccccc2c1)C(C)(O)c1ccc2ccccc2c1</smiles>

\section{2,3-di(naphthalen-2-yl)butane-2,3-diol (2x) ${ }^{1}$}

This compound was prepared according to the general procedure under $9 \mathrm{~W}$ LEDs. Purified on silica gel chromatography (PE/EA 15/1 to 5/1) to give the product, $8 \mathrm{~h}$, yellow oil ( $85.0 \mathrm{mg}, 99 \%)$. meso/dl $1: 1.3 ;{ }^{1} \mathbf{H}$ NMR of (meso)- and $(\boldsymbol{d l})-\mathbf{2 x}\left(400 \mathrm{MHz}, \mathrm{CDCl}_{3}\right) \delta 7.88-7.67(\mathrm{~m}, 18.4 \mathrm{H}, 1.3 \times 8 \mathrm{H} d l$ and $8 \mathrm{H}$ meso), 7.51-7.45 (m, 9.2, $1.3 \times 4 \mathrm{H} \mathrm{dl}$ and $4 \mathrm{H}$ meso), $7.41(\mathrm{~d}, J=8.6 \mathrm{~Hz}, 2 \mathrm{H}, m e s o), 7.28(\mathrm{~d}, J$ $=9.0 \mathrm{~Hz}, 1.3 \times 2 \mathrm{H}, d l), 2.79(\mathrm{~s}, 1.3 \times 2 \mathrm{H}, d l), 2.37(\mathrm{~s}, 2 \mathrm{H}$, meso $), 1.70(\mathrm{~s}, 6 \mathrm{H}$, meso $), 1.64(\mathrm{~s}, 1.3 \times$ $6 \mathrm{H}, d l) .{ }^{13} \mathbf{C}$ NMR of (meso)- and $(d l)-2 \mathbf{x}\left(100 \mathrm{MHz}, \mathrm{CDCl}_{3}\right) \delta 141.6,141.1,132.8,132.7,132.6$, 132.5, 128.5, 128.4, 127.5 (2C), 126.9, 126.6, 126.6, 126.1, 126.0, 126.0, 125.9, 125.6, 79.4, 79.0, 
<smiles>CCOC(=O)C(O)(O)C(O)(O)C(=O)OCC</smiles>

\section{diethyl 2,3-dihydroxy-2,3-dimethylsuccinate $(2 y)^{1}$}

This compound was prepared according to the general procedure under $18 \mathrm{~W}$ LEDs in $4 \mathrm{mLCH}_{3} \mathrm{CN}$. Purified on silica gel chromatography (PE/EA 15/1 to 5/1) to give the product, $12 \mathrm{~h}$, yellow oil (34.1 $\mathrm{mg}, 58 \%)$. meso/dl 1:1; ${ }^{1} \mathrm{H}$ NMR of (meso)- and (dl)-2y $\left(400 \mathrm{MHz}, \mathrm{CDCl}_{3}\right) \delta 4.26(\mathrm{qd}, J=7.2 \mathrm{~Hz}$, $1.4 \mathrm{~Hz}, 4 \mathrm{H}, d l), 4.22(\mathrm{q}, J=7.1 \mathrm{~Hz}, 4 \mathrm{H}, m e s o), 3.86(\mathrm{~s}, 2 \mathrm{H}, d l), 3.61(\mathrm{~s}, 2 \mathrm{H}, m e s o), 1.51(\mathrm{~s}, 6 \mathrm{H}, d l)$, 1.49 (s, 6H, meso), 1.32 (t, $J=7.2 \mathrm{~Hz}, 6 \mathrm{H}, d l), 1.28\left(\mathrm{t}, J=7.2 \mathrm{~Hz}, 6 \mathrm{H}\right.$, meso). ${ }^{13} \mathrm{C}$ NMR of (meso)and $(d l)-2 \mathbf{y}\left(100 \mathrm{MHz}, \mathrm{CDCl}_{3}\right) \delta 174.9,174.4,78.7,78.6,62.5,62.4,20.6,20.5,14.2,14.1$.<smiles>c1ccc(CNC(NCc2ccccc2)c2ccccc2)cc1</smiles>

\section{$N^{1}, N^{2}$-dibenzyl-1,2-diphenylethane-1,2-diamine (4a) ${ }^{1}$}

This compound was prepared according to the general procedure using $\mathrm{Et}_{3} \mathrm{~N}(1.0 \mathrm{mmol}, 138 \mu \mathrm{L})$ under 18 W LEDs. Purified on aluminum oxide active neutral chromatography (PE/EA 15/1 to 5/1) to give the product, $12 \mathrm{~h}$, yellow solid $(69.8 \mathrm{mg}, 68 \%)$. meso/dl $1: 1.8 ;{ }^{1} \mathrm{H}$ NMR of (meso)- and $(d l)-4 \mathbf{a}\left(500 \mathrm{MHz}, \mathrm{CDCl}_{3}\right) \delta 7.36-7.14(\mathrm{~m}, 45 \mathrm{H}, 1.8 \times 16 \mathrm{H} d l$ and $16 \mathrm{H}$ meso $), 7.06(\mathrm{~d}, J=6.6 \mathrm{~Hz}$, $4 \mathrm{H}$, meso), $6.99(\mathrm{~d}, J=7.0 \mathrm{~Hz}, 1.8 \times 4 \mathrm{H}, d l), 3.77(\mathrm{~s}, 1.8 \times 2 \mathrm{H}, d l), 3.73(\mathrm{~s}, 2 \mathrm{H}$, meso $), 3.68(\mathrm{~d}, J=$ $13.3 \mathrm{~Hz}, 2 \mathrm{H}$, meso), $3.55(\mathrm{~d}, J=13.8 \mathrm{~Hz}, 1.8 \times 2 \mathrm{H}, d l), 3.51(\mathrm{~d}, J=13.3 \mathrm{~Hz}, 2 \mathrm{H}, m e s o), 3.31(\mathrm{~d}, J$ $=13.8 \mathrm{~Hz}, 1.8 \times 2 \mathrm{H}, d l), 1.92\left(\mathrm{br} \mathrm{s}, 5.6 \mathrm{H}, 1.8 \times 2 \mathrm{H} \mathrm{dl}\right.$ and $2 \mathrm{H}$ meso). ${ }^{13} \mathbf{C ~ N M R ~ o f ~ ( m e s o ) - ~ a n d ~ ( d l ) - ~}$ 4a $\left(125 \mathrm{MHz}, \mathrm{CDCl}_{3}\right) \delta 141.3,140.9,140.7,140.4,128.7,128.5$ (2C), 128.4, 128.3, 128.2, 128.1, $128.0,127.8,127.1,126.9,126.8,68.5,67.3,51.5,51.1$.<smiles>N#Cc1ccc(C(NCc2ccccc2)C(NCc2ccccc2)c2ccccc2)cc1</smiles>

\section{4,4'-(1,2-bis(benzylamino)ethane-1,2-diyl)dibenzonitrile (4b) ${ }^{1}$}

This compound was prepared according to the general procedure using $\mathrm{Et}_{3} \mathrm{~N}(1.0 \mathrm{mmol}, 138 \mu \mathrm{L})$ under 18 W LEDs. Purified on aluminum oxide active neutral chromatography (PE/EA 15/1 to 5/1) to give the product, $12 \mathrm{~h}$, yellow oil (90.8 mg, 82\%). meso/dl 1.2:1; ${ }^{1} \mathrm{H}$ NMR of (meso)- and (dl)4b $\left(500 \mathrm{MHz}, \mathrm{CDCl}_{3}\right) \delta 7.56(\mathrm{~d}, J=8.2 \mathrm{~Hz}, 4 \mathrm{H}, d l), 7.47$ (d, $J=8.2 \mathrm{~Hz}, 1.2 \times 4 \mathrm{H}$, meso), 7.32-7.18 $(\mathrm{m}, 22 \mathrm{H}, 10 \mathrm{H} \mathrm{dl}$ and $1.2 \times 10 \mathrm{H}$ meso), $7.12(\mathrm{~d}, J=8.1 \mathrm{~Hz}, 1.2 \times 4 \mathrm{H}, m e s o), 7.05(\mathrm{~d}, J=6.7 \mathrm{~Hz}, 4 \mathrm{H}$, $d l), 3.87(\mathrm{~s}, 2 \mathrm{H}, d l), 3.71(\mathrm{~s}, 1.2 \times 2 \mathrm{H}$, meso $), 3.63(\mathrm{~d}, J=13.3 \mathrm{~Hz}, 1.2 \times 2 \mathrm{H}$, meso), $3.58(\mathrm{~d}, J=$ $13.6 \mathrm{~Hz}, 2 \mathrm{H}, d l), 3.46(\mathrm{~d}, J=13.3 \mathrm{~Hz}, 1.2 \times 2 \mathrm{H}$, meso $), 3.34(\mathrm{~d}, J=13.6 \mathrm{~Hz}, 2 \mathrm{H}, d l), 2.09(\mathrm{~s}, 4.4 \mathrm{H}$, $2 \mathrm{H} d l$ and $1.2 \times 2 \mathrm{H} \mathrm{meso)} .{ }^{13} \mathbf{C ~ N M R ~ o f ~ ( m e s o ) - ~ a n d ~}(d)-4 \mathbf{b}\left(125 \mathrm{MHz}, \mathrm{CDCl}_{3}\right) \delta 146.5,145.7$, 139.6, 139.5, 132.2, 132.1, 129.3, 128.7, 128.6, 128.6, 128.1, 128.0, 127.4, 127.3, 118.8, 118.7, 111.7, 111.4, 67.9, 66.4, 51.4, 51.2. 
<smiles>CC(=O)c1ccc(C(NCc2ccccc2)C(Nc2ccccc2)c2ccc(C(C)=O)cc2)cc1</smiles>

\section{dimethyl 4,4'-(1,2-bis(benzylamino)ethane-1,2-diyl)dibenzoate (4c) ${ }^{1}$}

This compound was prepared according to the general procedure using $\mathrm{Et}_{3} \mathrm{~N}(1.0 \mathrm{mmol}, 138 \mu \mathrm{L})$ under 18 W LEDs. Purified on aluminum oxide active neutral chromatography (PE/EA 15/1 to 5/1) to give the product, $10 \mathrm{~h}$, yellow solid (94.7 mg, 74\%). meso/dl 1:1.15; ${ }^{1} \mathrm{H}$ NMR of (meso)- and $(d l)-4 \mathrm{c}\left(500 \mathrm{MHz}, \mathrm{CDCl}_{3}\right) \delta 7.97(\mathrm{~d}, J=8.0 \mathrm{~Hz}, 1.15 \times 4 \mathrm{H}, d l), 7.82(\mathrm{~d}, J=8.0 \mathrm{~Hz}, 4 \mathrm{H}, m e s o)$, 7.32-7.21 (m, 21.5H, $1.15 \times 10 \mathrm{H} d l$ and $10 \mathrm{H}$ meso), $7.09(\mathrm{~d}, J=8.0 \mathrm{~Hz}, 4 \mathrm{H}$, meso), $7.01(\mathrm{~d}, J=6.9$ $\mathrm{Hz}, 1.15 \times 4 \mathrm{H}, d l), 3.93(\mathrm{~s}, 1.15 \times 6 \mathrm{H}, d l), 3.88-3.87(\mathrm{~m}, 8.3 \mathrm{H}, 1.15 \times 2 \mathrm{H} d l$ and $6 \mathrm{H} \mathrm{meso}), 3.75(\mathrm{~s}$, $2 \mathrm{H}, m e s o), 3.63(\mathrm{~d}, J=13.3 \mathrm{~Hz}, 2 \mathrm{H}, m e s o), 3.56(\mathrm{~d}, J=13.7 \mathrm{~Hz}, 1.15 \times 2 \mathrm{H}, d l), 3.48(\mathrm{~d}, J=13.3$ $\mathrm{Hz}, 2 \mathrm{H}$, meso $), 3.32(\mathrm{~d}, J=13.7 \mathrm{~Hz}, 1.15 \times 2 \mathrm{H}, d l), 2.01(\mathrm{~s}, 4.3 \mathrm{H}, 1.15 \times 2 \mathrm{H} d l$ and $2 \mathrm{H} \mathrm{meso}) .{ }^{13} \mathrm{C}$ NMR of (meso)- and (dl)-4c (125 MHz, $\left.\mathrm{CDCl}_{3}\right) \delta 167.1,167.0,146.5,145.9,140.2,139.9,129.7$ (2C), 129.6, 129.2, 128.6, 128.5, 128.5, 128.2, 128.1, 128.1, 127.1, 127.1, 68.1, 66.7, 52.3, 52.2, $51.4,51.2$.<smiles>Cc1ccccc1C(NCc1ccccc1)C(NBr)c1ccccc1</smiles>

\section{$N^{1}, N^{2}$-dibenzyl-1,2-di-o-tolylethane-1,2-diamine $(4 \mathrm{~d})^{1}$}

This compound was prepared according to the general procedure using $\mathrm{Et}_{3} \mathrm{~N}(1.0 \mathrm{mmol}, 138 \mu \mathrm{L})$ under 18 W LEDs. Purified on aluminum oxide active neutral chromatography (PE/EA 15/1 to 3/1) to give the product, $12 \mathrm{~h}$, yellow oil (83.6 mg, 80\%). meso/dl 1.1:1; ${ }^{1} \mathrm{H}$ NMR of (meso)- and (dl)$4 \mathbf{d}\left(400 \mathrm{MHz}, \mathrm{CDCl}_{3}\right) \delta 7.59(\mathrm{~d}, J=7.7 \mathrm{~Hz}, 1.1 \times 2 \mathrm{H}$, meso $), 7.36-7.04(\mathrm{~m}, 33.4 \mathrm{H}, 18 \mathrm{H} d l$ and 1.1 $\times 14 \mathrm{H} \mathrm{meso}), 6.88(\mathrm{~d}, J=7.4 \mathrm{~Hz}, 1.1 \times 2 \mathrm{H}$, meso $), 4.20(\mathrm{~s}, 2 \mathrm{H}, d l), 4.09(\mathrm{~s}, 1.1 \times 2 \mathrm{H}$, meso $), 3.66$ $(\mathrm{d}, J=13.3 \mathrm{~Hz}, 1.1 \times 2 \mathrm{H}$, meso $), 3.60(\mathrm{~d}, J=13.8 \mathrm{~Hz}, 2 \mathrm{H}$, meso $), 3.50(\mathrm{~d}, J=13.3 \mathrm{~Hz}, 1.1 \times 2 \mathrm{H}$, $m e s o), 3.36(\mathrm{~d}, J=13.8 \mathrm{~Hz}, 2 \mathrm{H}, d l), 2.24-2.04(\mathrm{~m}, 10.8 \mathrm{H}, 2 \mathrm{H} d l$ and $1.1 \times 8 \mathrm{H} \mathrm{meso}), 1.77(\mathrm{~s}, 6 \mathrm{H}$, $d l) .{ }^{13} \mathbf{C}$ NMR of (meso)- and $(\boldsymbol{d l})-\mathbf{4 d}\left(100 \mathrm{MHz}, \mathrm{CDCl}_{3}\right) \delta 140.9,140.8,139.7(2 \mathrm{C}), 137.8,137.2$, 130.0 (2C), 128.4, 128.3, 128.2, 128.0, 127.2 (2C), 127.0, 126.9, 126.8, 126.7, 126.2, 125.9, 62.5 (2C), 51.2, 50.9, 19.5, 19.4 .<smiles>Fc1ccccc1C(NCc1ccccc1)C(Nc1ccccc1)c1ccccc1</smiles>

\section{$N^{1}, N^{2}$-dibenzyl-1,2-bis(2-fluorophenyl)ethane-1,2-diamine (4e) $)^{1}$}

This compound was prepared according to the general procedure using $\mathrm{Et}_{3} \mathrm{~N}(1.0 \mathrm{mmol}, 138 \mu \mathrm{L})$ under 18 W LEDs. Purified on aluminum oxide active neutral chromatography (PE/EA 15/1 to 5/1) to give the product, $12 \mathrm{~h}$, yellow solid $(100.9 \mathrm{mg}, 94 \%)$. meso/dl 1.1:1; ${ }^{1} \mathrm{H}$ NMR of (meso)- and $(d l)-4 \mathrm{e}\left(500 \mathrm{MHz}, \mathrm{CDCl}_{3}\right) \delta 7.31-7.20(\mathrm{~m}, 23 \mathrm{H}, 12 \mathrm{H} d l$ and $1.1 \times 10 \mathrm{H}$ meso $), 7.13-7.12(\mathrm{~m}, 4.2 \mathrm{H}$, 
$2 \mathrm{H} d l$ and $1.1 \times 2 \mathrm{H} \mathrm{meso}), 7.10-7.04(\mathrm{~m}, 4.2 \mathrm{H}, 2 \mathrm{H} d l$ and $1.1 \times 2 \mathrm{H} \mathrm{meso}), 7.00-6.94(\mathrm{~m}, 4.2 \mathrm{H}, 2 \mathrm{H}$ $d l$ and $1.1 \times 2 \mathrm{H} \mathrm{meso}), 6.79(\mathrm{t}, J=9.5 \mathrm{~Hz}, 1.1 \times 2 \mathrm{H} \mathrm{meso}), 4.35(\mathrm{~s}, 2 \mathrm{H}, d l), 4.18(\mathrm{~s}, 1.1 \times 2 \mathrm{H}$, meso $)$, $3.64(\mathrm{~d}, J=13.3 \mathrm{~Hz}, 4.2 \mathrm{H}, 2 \mathrm{H} d l$ and $1.1 \times 2 \mathrm{H}$ meso), $3.55(\mathrm{~d}, J=13.2 \mathrm{~Hz}, 1.1 \times 2 \mathrm{H}$, meso $), 3.46$ $(\mathrm{d}, J=13.5 \mathrm{~Hz}, 2 \mathrm{H}, d l), 2.20(\mathrm{~s}, 4.2 \mathrm{H}, 2 \mathrm{H} d l$ and $1.1 \times 2 \mathrm{H}$ meso $) .{ }^{13} \mathbf{C}$ NMR of (meso)- and $(d l)-4 \mathrm{e}$ $\left(125 \mathrm{MHz}, \mathrm{CDCl}_{3}\right) \delta 161.7\left(\mathrm{C}-\mathrm{F}, 1 J_{\mathrm{C}-\mathrm{F}}=243.9 \mathrm{~Hz}\right), 161.4\left(\mathrm{C}-\mathrm{F}, 1 J_{\mathrm{C}-\mathrm{F}}=244.0 \mathrm{~Hz}\right), 140.5,140.4$, $129.5\left(\mathrm{C}-\mathrm{F}, 4 J_{\mathrm{C}-\mathrm{F}}=3.7 \mathrm{~Hz}\right), 129.0\left(\mathrm{C}-\mathrm{F}, 4 J_{\mathrm{C}-\mathrm{F}}=3.7 \mathrm{~Hz}\right), 128.7\left(\mathrm{C}-\mathrm{F}, 3 J_{\mathrm{C}-\mathrm{F}}=8.2 \mathrm{~Hz}\right), 128.7(\mathrm{C}-\mathrm{F}$, $\left.3 J_{\mathrm{C}-\mathrm{F}}=8.2 \mathrm{~Hz}\right), 128.5,128.4,128.3,128.1,128.0\left(\mathrm{C}-\mathrm{F}, 2 J_{\mathrm{C}-\mathrm{F}}=28.8 \mathrm{~Hz}\right), 127.8\left(\mathrm{C}-\mathrm{F}, 2 J_{\mathrm{C}-\mathrm{F}}=29.1\right.$ $\mathrm{Hz}), 127.0,126.9,124.0,124.0,115.2\left(\mathrm{C}-\mathrm{F}, 2 J_{\mathrm{C}-\mathrm{F}}=22.6 \mathrm{~Hz}\right), 115.1\left(\mathrm{C}-\mathrm{F}, 2 J_{\mathrm{C}-\mathrm{F}}=22.7 \mathrm{~Hz}\right), 61.2$, 58.7, 51.7, 51.4. ${ }^{19} \mathbf{F}$ NMR of (meso)- and $(\boldsymbol{d l})-4 \mathbf{e}\left(471 \mathrm{MHz}, \mathrm{CDCl}_{3}\right.$, decoupled) $\delta$-118.3, -118.6.<smiles>FC(F)(F)c1cccc(C(NCc2ccccc2)C(Nc2ccccc2)c2cccc(C(F)(F)F)c2)c1</smiles>

\section{$N^{\boldsymbol{I}}, N^{2}$-dibenzyl-1,2-bis(3-(trifluoromethyl)phenyl)ethane-1,2-diamine (4f)}

This compound was prepared according to the general procedure using $\mathrm{Et}_{3} \mathrm{~N}(1.0 \mathrm{mmol}, 138 \mu \mathrm{L})$ under 18 W LEDs. Purified on aluminum oxide active neutral chromatography (PE/EA 20/1 to 10/1) to give the product, $12 \mathrm{~h}$, yellow solid (105.9 mg, 80\%). meso/dl 1:1.35; ${ }^{1} \mathbf{H}$ NMR of (meso)- and (dl)-4f $\left(500 \mathrm{MHz}, \mathrm{CDCl}_{3}\right) \delta 7.55(\mathrm{~d}, J=7.5 \mathrm{~Hz}, 1.35 \times 2 \mathrm{H}, d l), 7.42-7.19(\mathrm{~m}, 34.2 \mathrm{H}$, $1.35 \times 12 \mathrm{H} d l$ and $18 \mathrm{H} \mathrm{meso}$ ), $7.01(\mathrm{~d}, J=7.2 \mathrm{~Hz}, 1.35 \times 4 \mathrm{H}, d l), 3.85(\mathrm{~s}, 1.35 \times 2 \mathrm{H}, d l), 3.73(\mathrm{~s}$, $2 \mathrm{H}, m e s o), 3.66(\mathrm{~d}, J=13.3 \mathrm{~Hz}, 2 \mathrm{H}, m e s o), 3.58(\mathrm{~d}, J=13.8 \mathrm{~Hz}, 1.35 \times 2 \mathrm{H}, d l), 3.49(\mathrm{~d}, J=13.3$ $\mathrm{Hz}, 2 \mathrm{H}$, meso), $3.32(\mathrm{~d}, J=13.8 \mathrm{~Hz}, 1.35 \times 2 \mathrm{H}, d l), 2.06(\mathrm{~s}, 4.7 \mathrm{H}, 1.35 \times 2 \mathrm{H} \mathrm{dl}$ and $2 \mathrm{H} \mathrm{meso}) .{ }^{13} \mathrm{C}$ NMR of (meso)- and (dl)-4f (125 MHz, $\left.\mathrm{CDCl}_{3}\right) \delta 142.0,141.4,140.0,139.8,132.1,131.1,130.8$ $\left(\mathrm{C}-\mathrm{F}, 2 J_{\mathrm{C}-\mathrm{F}}=31.8 \mathrm{~Hz}\right), 130.6\left(\mathrm{C}-\mathrm{F}, 2 J_{\mathrm{C}-\mathrm{F}}=32.0 \mathrm{~Hz}\right), 128.8,128.7,128.6,128.5,128.2,128.0$, 127.2, 127.2, $125.3\left(\mathrm{C}-\mathrm{F}, 3 J_{\mathrm{C}-\mathrm{F}}=3.6 \mathrm{~Hz}\right), 124.8\left(\mathrm{C}-\mathrm{F}, 3 J_{\mathrm{C}-\mathrm{F}}=3.7 \mathrm{~Hz}\right), 124.7\left(\mathrm{C}-\mathrm{F}, 3 J_{\mathrm{C}-\mathrm{F}}=3.7 \mathrm{~Hz}\right)$ $124.2\left(\mathrm{C}-\mathrm{F}, 3 J_{\mathrm{C}-\mathrm{F}}=3.7 \mathrm{~Hz}\right), 124.2\left(\mathrm{C}-\mathrm{F}, 1 J_{\mathrm{C}-\mathrm{F}}=270.7 \mathrm{~Hz}\right), 124.1\left(\mathrm{C}-\mathrm{F}, 1 J_{\mathrm{C}-\mathrm{F}}=270.9 \mathrm{~Hz}\right), 68.3$, 66.4, 51.5, 51.1. ${ }^{19} \mathbf{F}$ NMR of (meso)- and (dl) $-4 \mathbf{f}\left(471 \mathrm{MHz}, \mathrm{CDCl}_{3}\right.$, decoupled) $\delta-62.5,-62.7$.<smiles>N#Cc1ccccc1NC(c1ccc(Cl)cc1)C(Nc1ccccc1C#N)c1ccc(Cl)cc1</smiles>

\section{2,2'-((1,2-bis(4-chlorophenyl)ethane-1,2-diyl)bis(azanediyl))dibenzonitrile (4g) ${ }^{2}$}

This compound was prepared according to the general procedure using $\mathrm{Et}_{3} \mathrm{~N}(1.0 \mathrm{mmol}, 138 \mu \mathrm{L})$ under 18 W LEDs. Purified on aluminum oxide active neutral chromatography (PE/EA 30/1 to 10/1) to give the product, $12 \mathrm{~h}$, yellow oil (99.2 mg, 82\%). meso/dl 1:1; ${ }^{1} \mathbf{H}$ NMR of (meso)- and (dl)-4g $\left(500 \mathrm{MHz}, \mathrm{CDCl}_{3}\right) \delta 7.40(\mathrm{t}, J=6.1 \mathrm{~Hz}, 4 \mathrm{H}), 7.31-7.27(\mathrm{~m}, 8 \mathrm{H}), 7.23(\mathrm{t}, J=7.9 \mathrm{~Hz}, 4 \mathrm{H}), 7.05$ (d, $J=8.3 \mathrm{~Hz}, 4.0 \mathrm{H}), 6.99(\mathrm{t}, J=8.3 \mathrm{~Hz}, 4 \mathrm{H}), 6.71(\mathrm{t}, J=7.5 \mathrm{~Hz}, 4 \mathrm{H}), 6.40-6.35(\mathrm{~m}, 4 \mathrm{H}), 5.30-5.28$ $(\mathrm{m}, 4 \mathrm{H}$, meso $), 4.96(\mathrm{~d}, J=6.6 \mathrm{~Hz}, 2 \mathrm{H}, d l), 4.73-4.71(\mathrm{~m}, 2 \mathrm{H}, d l) .{ }^{13} \mathrm{C}$ NMR of (meso)- and (dl)$4 \mathrm{~g}\left(125 \mathrm{MHz}, \mathrm{CDCl}_{3}\right) \delta 148.4,148.2,135.8,134.9,134.7,134.6,134.3,134.3,133.0,132.9,129.4$, 
<smiles>Clc1ccc(C(Nc2ccccc2)C(Nc2ccccc2)c2ccccc2)cc1</smiles>

\section{1,2-bis(4-chlorophenyl)- $N^{1}, N^{2}$-diphenylethane-1,2-diamine (4h) ${ }^{2}$}

This compound was prepared according to the general procedure using $\mathrm{Et}_{3} \mathrm{~N}(1.0 \mathrm{mmol}, 138 \mu \mathrm{L})$ under 18 W LEDs. Purified on aluminum oxide active neutral chromatography (PE/EA 30/1 to 10/1) to give the product, $12 \mathrm{~h}$, yellow solid (93.3 mg, 86\%). meso/dl 1.3:1; ${ }^{1} \mathbf{H}$ NMR of (meso)-4h (500 $\left.\mathrm{MHz}, \mathrm{CDCl}_{3}\right) \delta 7.23(\mathrm{~d}, J=8.2 \mathrm{~Hz}, 4 \mathrm{H}), 7.12(\mathrm{t}, J=7.7 \mathrm{~Hz}, 4 \mathrm{H}), 6.91(\mathrm{~d}, J=8.2 \mathrm{~Hz}, 4 \mathrm{H}), 6.71(\mathrm{t}$, $J=7.3 \mathrm{~Hz}, 2 \mathrm{H}), 6.51(\mathrm{~d}, J=8.0 \mathrm{~Hz}, 4 \mathrm{H}), 4.94(\mathrm{~d}, J=6.8 \mathrm{~Hz}, 2 \mathrm{H}), 4.48(\mathrm{~d}, J=7.2 \mathrm{~Hz}, 2 \mathrm{H}) .{ }^{13} \mathrm{C}$ NMR of (meso)-4h $\left(125 \mathrm{MHz}, \mathrm{CDCl}_{3}\right) \delta 146.0,136.6,133.7,129.4,128.9,128.8,118.4,113.9$, 61.4. ${ }^{1} \mathbf{H}$ NMR of $(\boldsymbol{d l})-4 \mathbf{h}\left(500 \mathrm{MHz}, \mathrm{CDCl}_{3}\right) \delta 7.20(\mathrm{~d}, J=8.2 \mathrm{~Hz}, 4 \mathrm{H}), 7.10(\mathrm{t}, J=7.7 \mathrm{~Hz}, 4 \mathrm{H})$, $7.05(\mathrm{~d}, J=8.2 \mathrm{~Hz}, 4 \mathrm{H}), 6.72(\mathrm{t}, J=7.3 \mathrm{~Hz}, 2 \mathrm{H}), 6.50(\mathrm{~d}, J=8.0 \mathrm{~Hz}, 4 \mathrm{H}), 4.56(\mathrm{~s}, 2 \mathrm{H}), 4.48(\mathrm{~s}, 2 \mathrm{H})$. ${ }^{13} \mathbf{C ~ N M R ~ o f ~}(\boldsymbol{d l})-4 \mathbf{h}\left(125 \mathrm{MHz}, \mathrm{CDCl}_{3}\right) \delta$ 146.6, 138.3, 133.5, 129.4, $128.8(2 \mathrm{C}), 118.7,114.2,63.6$.<smiles>OC(c1ccccc1)C(Nc1ccccc1)c1ccc(Cl)cc1</smiles>

\section{2-(4-chlorophenyl)-1-phenyl-2-(phenylamino)ethan-1-ol (5) ${ }^{8}$}

This compound was prepared according to the general procedure with CBZ6 (1 mol\%), 1-(4chlorophenyl)- $N$-phenylmethanimine $(0.5 \mathrm{mmol})$, benzaldehyde $(0.5 \mathrm{mmol}), \mathrm{Et}_{3} \mathrm{~N}(1.0 \mathrm{mmol})$ under $18 \mathrm{~W}$ LEDs in $2 \mathrm{~mL} \mathrm{CH}_{3} \mathrm{CN}$. Purified on aluminum oxide active neutral chromatography (PE/EA $30 / 1$ to $10 / 1)$ to give the product, $12 \mathrm{~h}$, yellow oil $(55.3 \mathrm{mg}, 34 \%)$. The diastereomeric ratio is $1: 1.1$ determined by ${ }^{1} \mathrm{H}$ NMR. ${ }^{1} \mathbf{H}$ NMR of $5\left(400 \mathrm{MHz}, \mathrm{CDCl}_{3}\right) \delta$ 7.30-7.29 (m, 3H), 7.24-7.19 (m, 3H), 7.14-7.12 (m, 1H), 7.01-7.04 (m, 4H), $6.68(\mathrm{t}, J=7.3 \mathrm{~Hz}, 1 \mathrm{H}), 6.52-6.49(\mathrm{~m}, 2 \mathrm{H}), 5.08$ (brs, $0.47 \times$ $1 \mathrm{H}), 4.81(\mathrm{~d}, J=6.2 \mathrm{~Hz}, 0.52 \times 1 \mathrm{H}), 4.63(\mathrm{~d}, J=4.5 \mathrm{~Hz}, 0.47 \times 1 \mathrm{H}), 4.50(\mathrm{~d}, J=6.2 \mathrm{~Hz}, 0.52 \times$ $1 \mathrm{H}), 2.48$ (brs, $0.47 \times 1 \mathrm{H}), 2.26$ (brs, $0.52 \times 1 \mathrm{H}) .{ }^{13} \mathbf{C} \mathbf{~ N M R}$ of $5\left(100 \mathrm{MHz}, \mathrm{CDCl}_{3}\right) \delta 147.2,146.6$, 140.4, 140.0, 138.9, 137.1, 133.4, 133.3, 129.5 (2C), 129.3, 129.2, 128.9, 128.8, 128.5, 128.5, 128.5, $128.3,126.8,126.6,118.3,118.2,114.3,114.0,78.2,77.1,64.4,63.2$. 


\section{Fluorescence and Luminescence Experiments}

Test conditions for quenching reaction:

CBZ6: $4.3 \mathrm{mg}$ dissolved in $10 \mathrm{~mL}$ DCM $(0.001 \mathrm{M})$

Quencher: $102 \mu \mathrm{L}$ of $\mathbf{1 a}$ dissolved in $5 \mathrm{~mL}$ DCM $(0.2 \mathrm{M})$.

General procedure:

$0.5 \mathrm{~mL}$ of prepared solution containing CBZ6 was added to a cuvette, keep the total volume at

$2 \mathrm{~mL}, \mathbf{1 a}$ and DCM were added as the following table:

\begin{tabular}{|c|c|c|c|c|}
\hline Entry & CBZ6 & 1a & DCM & Total volume \\
\hline 1 & $0.5 \mathrm{~mL}\left(2.5 \times 10^{-4} \mathrm{M}\right)$ & $0 \mathrm{~mL}(0 \mathrm{M})$ & $1.5 \mathrm{~mL}$ & $2 \mathrm{~mL}$ \\
\hline 2 & $0.5 \mathrm{~mL}\left(2.5 \times 10^{-4} \mathrm{M}\right)$ & $0.25 \mathrm{~mL}(25 \mathrm{mM})$ & $1.25 \mathrm{~mL}$ & $2 \mathrm{~mL}$ \\
\hline 3 & $0.5 \mathrm{~mL}\left(2.5 \times 10^{-4} \mathrm{M}\right)$ & $0.375 \mathrm{~mL}(37.5 \mathrm{mM})$ & $1.125 \mathrm{~mL}$ & $2 \mathrm{~mL}$ \\
\hline 4 & $0.5 \mathrm{~mL}\left(2.5 \times 10^{-4} \mathrm{M}\right)$ & $0.5 \mathrm{~mL}(50 \mathrm{mM})$ & $1 \mathrm{~mL}$ & $2 \mathrm{~mL}$ \\
\hline 5 & $0.5 \mathrm{~mL}\left(2.5 \times 10^{-4} \mathrm{M}\right)$ & $0.625 \mathrm{~mL}(62.5 \mathrm{mM})$ & $0.875 \mathrm{~mL}$ & $2 \mathrm{~mL}$ \\
\hline
\end{tabular}

Excitation wavelength: $330 \mathrm{~nm}$

Make and model of fluorescence spectrophotometer:

Make: Hitachi High-Technologies Corporation, Tokyo, Japan

Model: F-4600

\section{References}

1. Nakajima, M.; Fava, E.; Loesscher, S.; Jiang, Z.; Rueping, M. Photoredox-Catalyzed Reductive Coupling of Aldehydes, Ketones, and Imines with Visible Light. Angew. Chem. Int. Ed. 2015, 54, 8828-8832.

2. Gualandi, A.; Rodeghiero, G.; Della Rocca, E.; Bertoni, F.; Marchini, M.; Perciaccante, R.; Jansen, T. P.; Ceroni, P.; Cozzi, P. G. Application of coumarin dyes for organic photoredox catalysis. Chem. Commun. 2018, 54, 10044-10047.

3. Kim, Y. J., Kim, S. M., Hosono, H., Yang, J. W., Kim, S. W. The scalable pinacol coupling reaction utilizing the inorganic electride $\left[\mathrm{Ca}_{2} \mathrm{~N}\right]^{+} \cdot \mathrm{e}^{-}$as an electron donor. Chem. Commun., 2014, 50, 4791-4794.

4. Kronenwetter, H., Husek, J., Etz, B., Jones, A., Manchanayakage, R. Electrochemical pinacol coupling of aromatic carbonyl compounds in a $[\mathrm{BMIM}]\left[\mathrm{BF}_{4}\right]-\mathrm{H}_{2} \mathrm{O}$ mixture. Green Chem., 2014, 16, 1489-1495.

5. Wang, C.-Y., Pan, Y.-J., Wu, A.-X. InCl3/Al mediated pinacol coupling reactions of aldehydes and ketones in aqueous media. Tetrahedron 2007, 63, 429-434.

6. Guo, H.-Y., Zhang, Y.-M. A novel method for pinacol coupling of aromatic aldehydes and ketones with a Sm- $\mathrm{ZnCl}_{2}$ system. J. Chem. Research (S), 2000, 284-286.

7. Oku, A., Takahashi, H., Asmus, S. M. Sequential Two-Electron Transfer from TetraoxySubstituted Cyclopropane to Acceptors. J. Am. Chem. Soc. 2000, 122, 7388-7389.

8. Regati, S.; He, Y.-B.; Thimmaiah, M.; Li, P.; Xiang, S.-C.; Chen, B.-L.; Zhao, J. C-G. Chem. Commun. 2013, 49, 9836-9838. 


\section{NMR spectra}

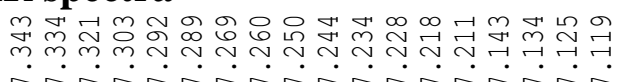

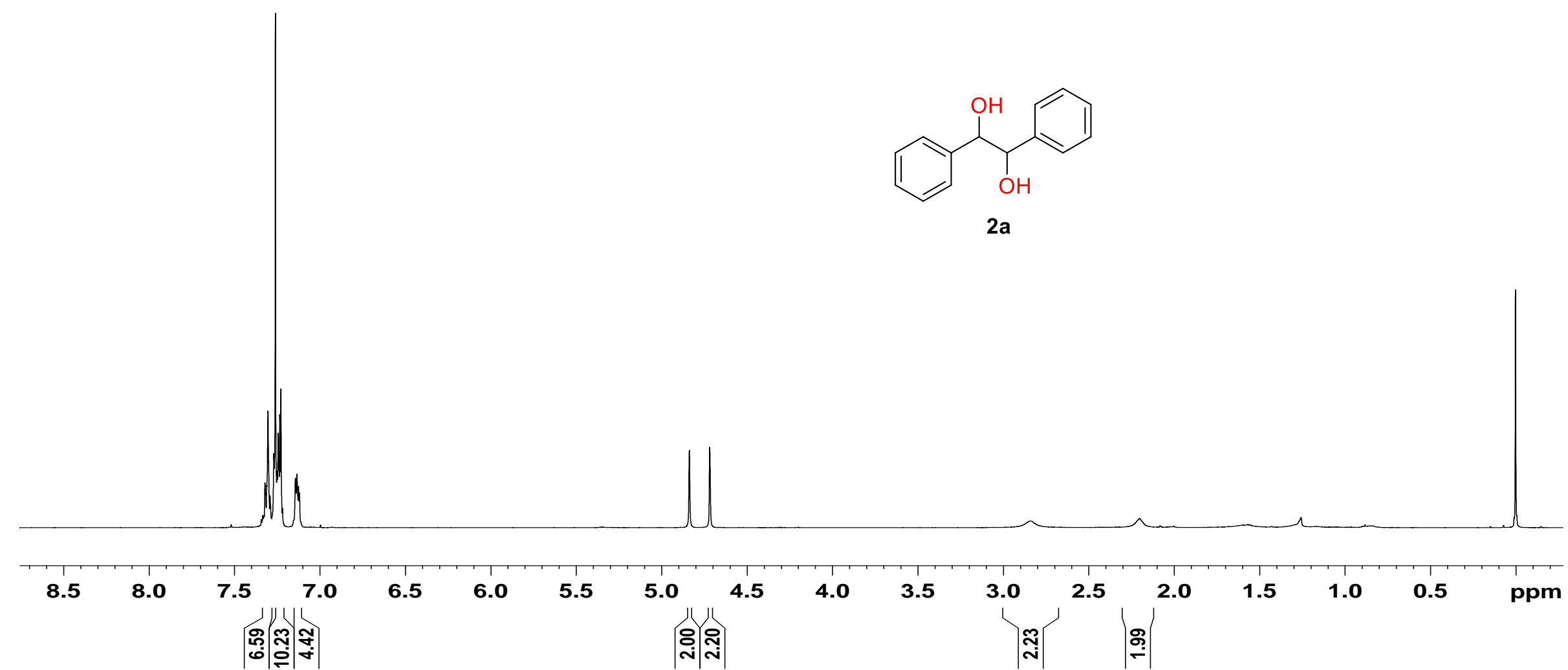

Figure S1. ${ }^{1} \mathrm{H}$ NMR spectra of $\mathbf{2 a}\left(\mathrm{CDCl}_{3}, 400 \mathrm{M}\right)$. 

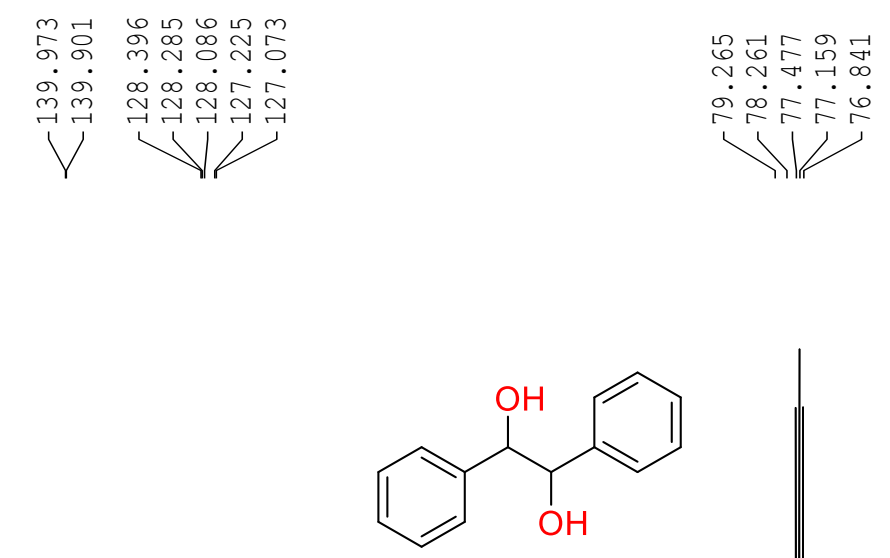

$2 a$

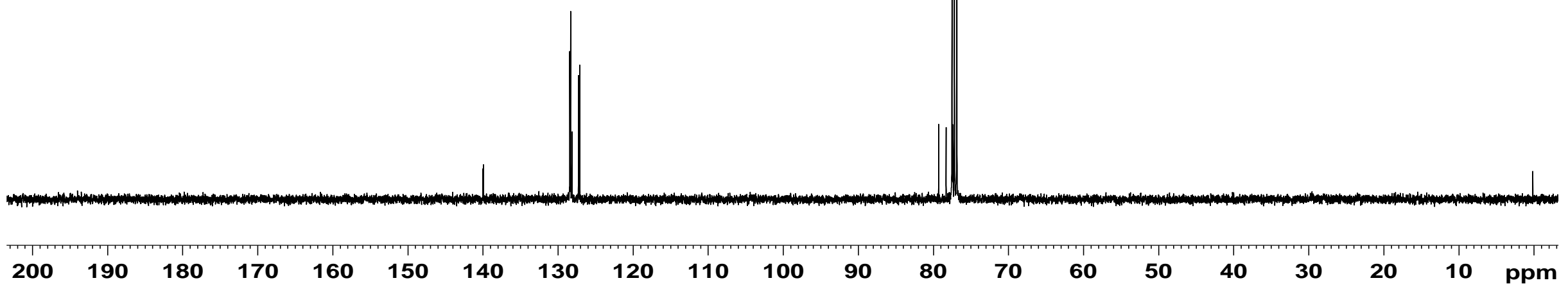

Figure S2. ${ }^{13} \mathrm{C}$ NMR spectra of $\mathbf{2 a}\left(\mathrm{CDCl}_{3}, 100 \mathrm{M}\right)$. 


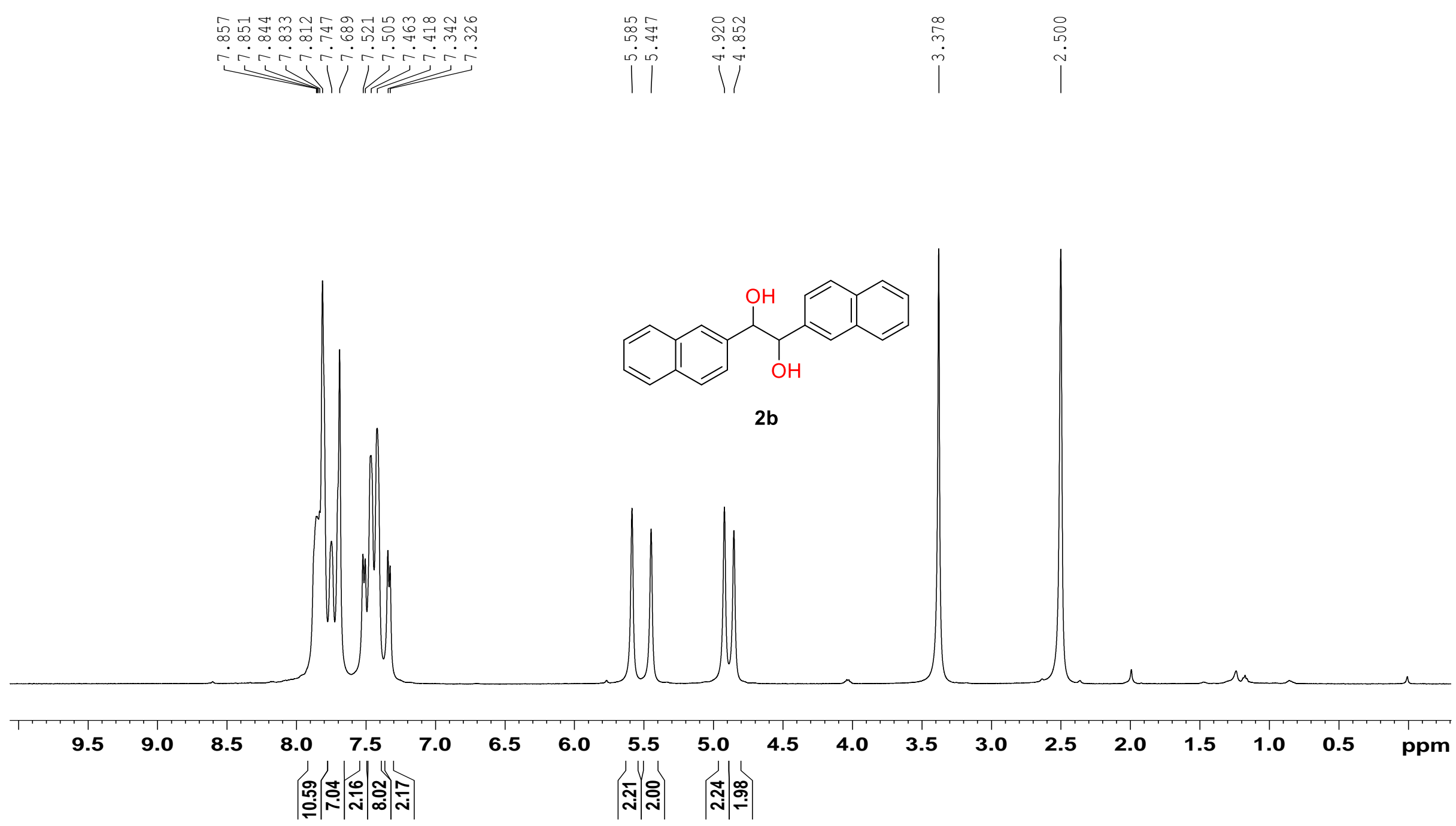

Figure S3. ${ }^{1} \mathrm{H}$ NMR spectra of $\mathbf{2 b}\left(d^{6}\right.$-DMSO, 500M). 

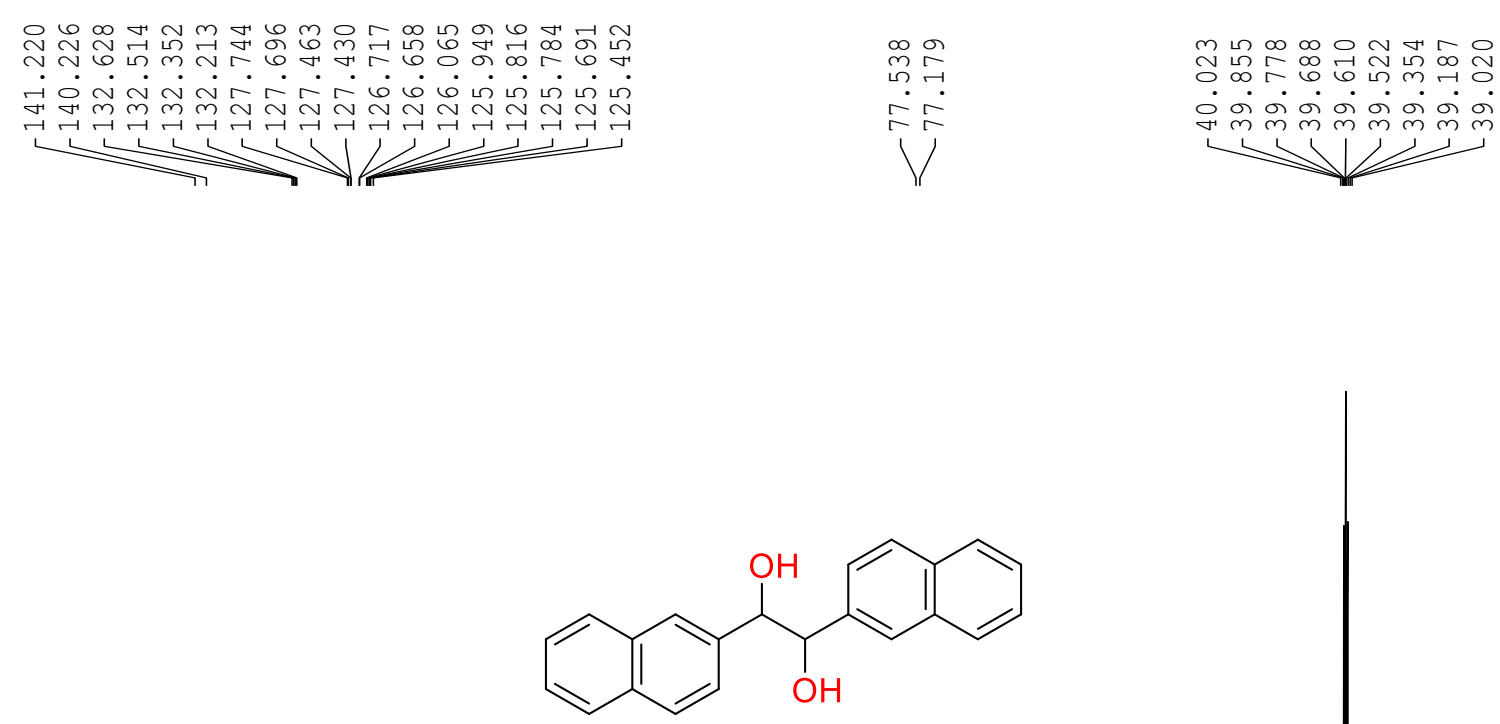

2b

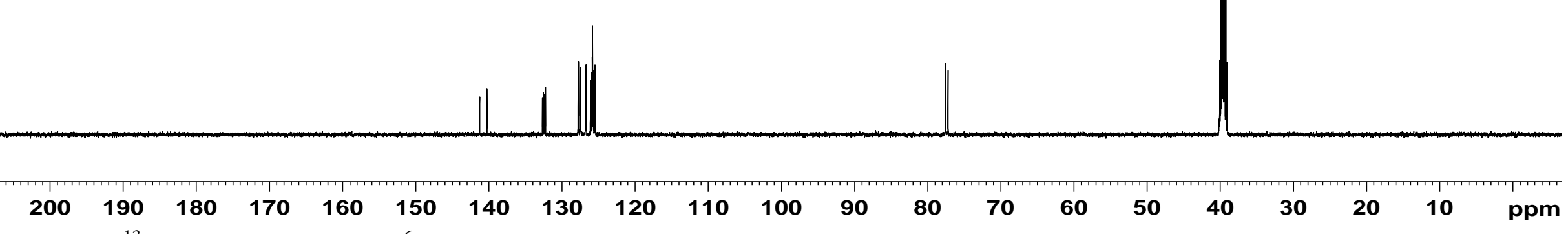

Figure $\mathbf{S 4} .{ }^{13} \mathrm{C}$ NMR spectra of $\mathbf{2 b}\left(d^{6}\right.$-DMSO, 125M) 


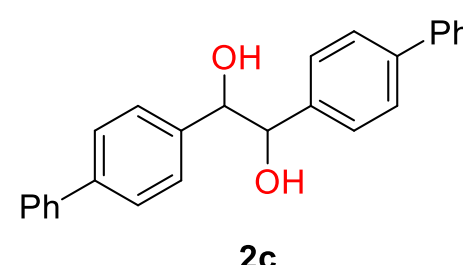

2c
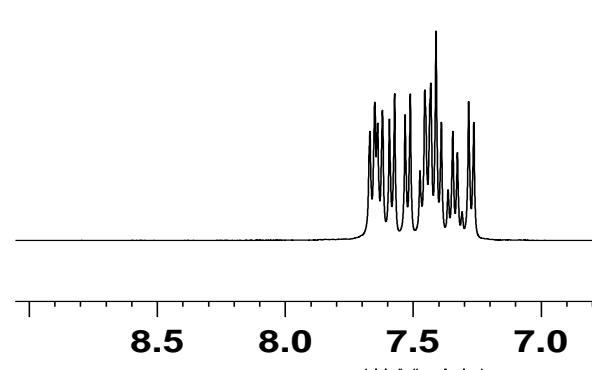

6.5

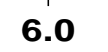

5.5

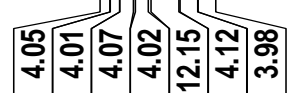

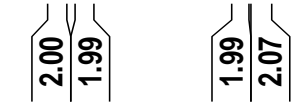

Figure S5. ${ }^{1} \mathrm{H}$ NMR spectra of $2 \mathrm{c}$ ( $d^{6}$-DMSO, 400M). 

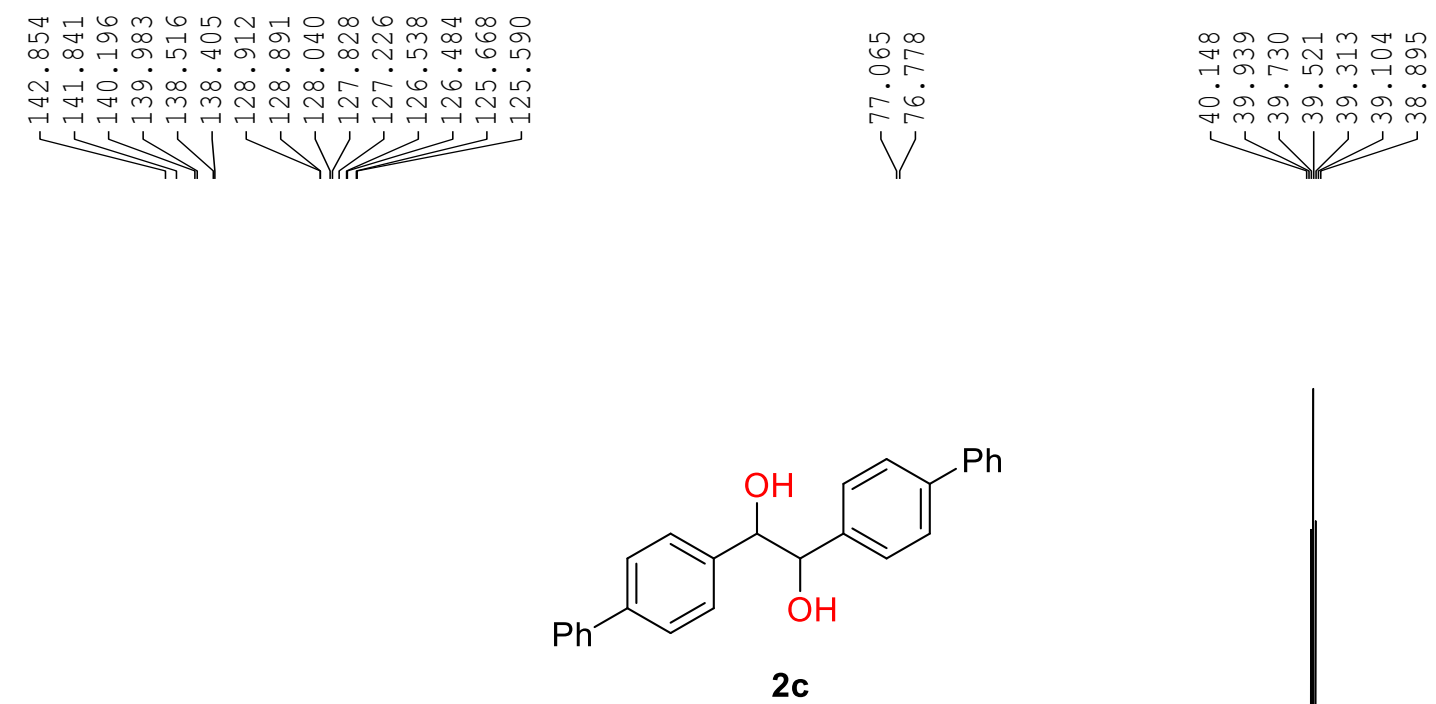

2c

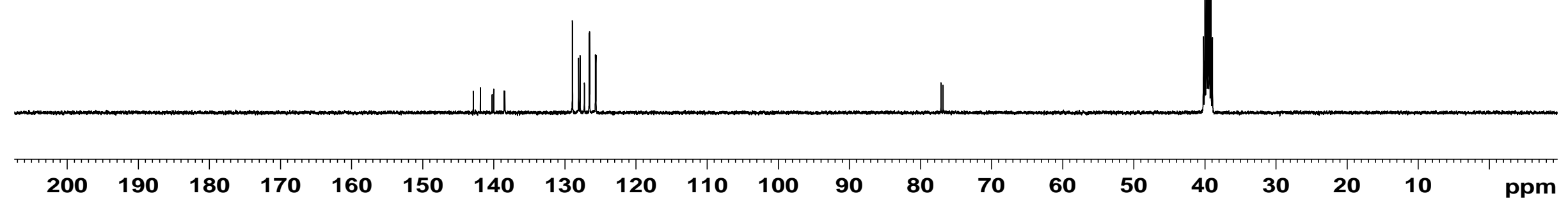

Figure S6. ${ }^{13} \mathrm{C}$ NMR spectra of $2 \mathrm{c}\left(d^{6}\right.$-DMSO, 100M). 


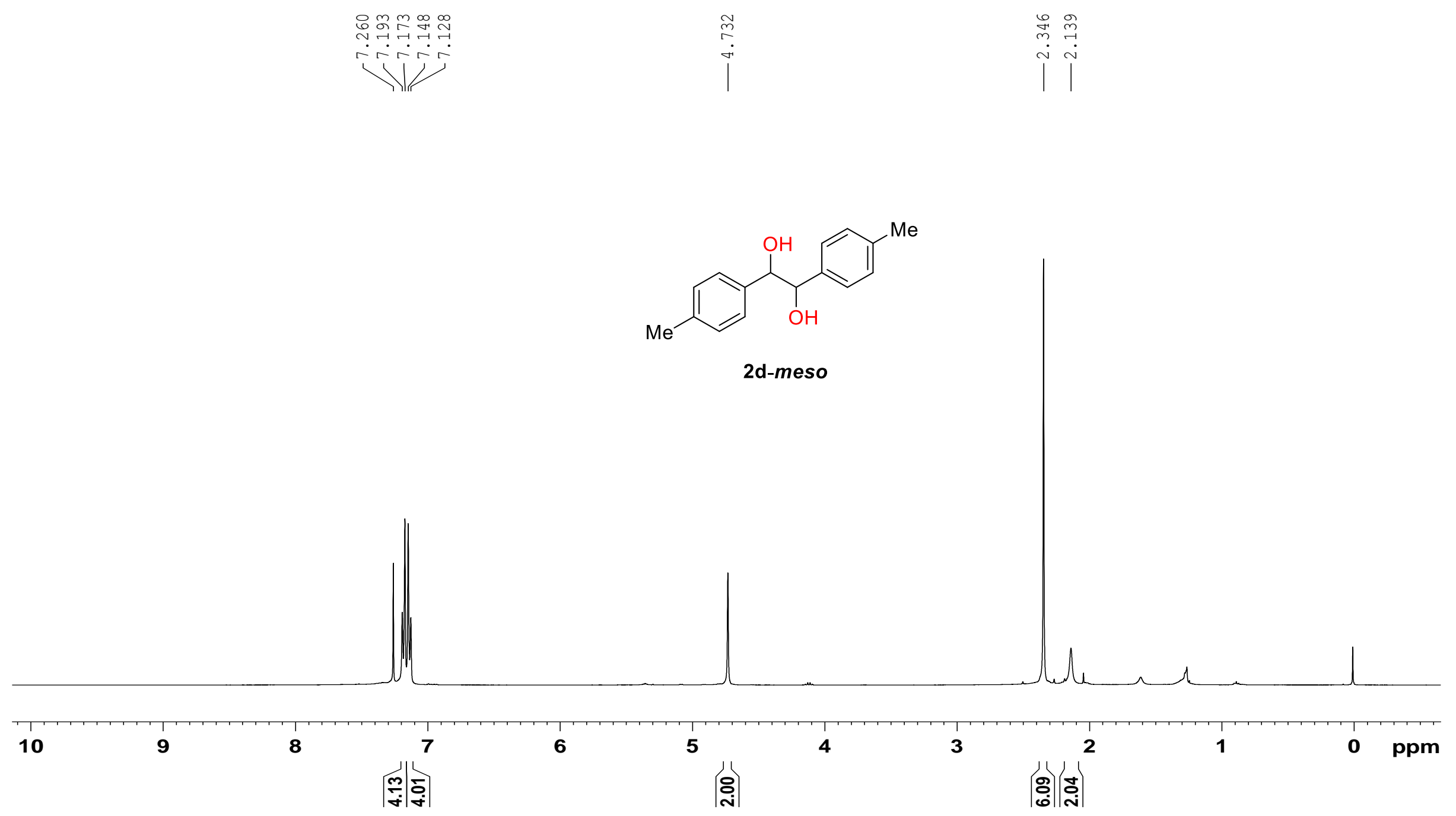

Figure S7. ${ }^{1} \mathrm{H}$ NMR spectra of $2 d-m e s o\left(\mathrm{CDCl}_{3}, 400 \mathrm{M}\right)$. 


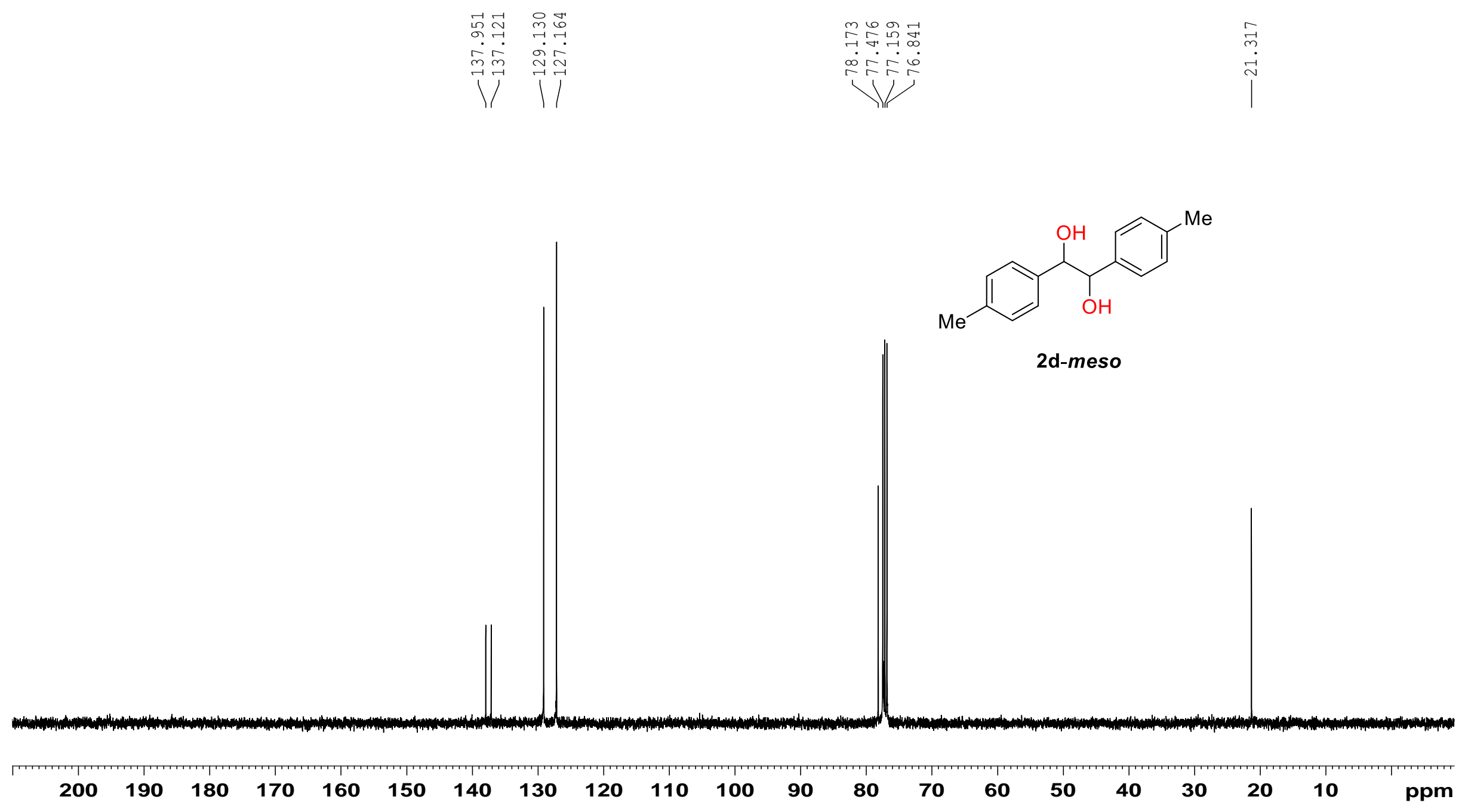

Figure S8. ${ }^{13} \mathrm{C}$ NMR spectra of $\mathbf{2 d}$-meso $\left(\mathrm{CDCl}_{3}, 100 \mathrm{M}\right)$. 

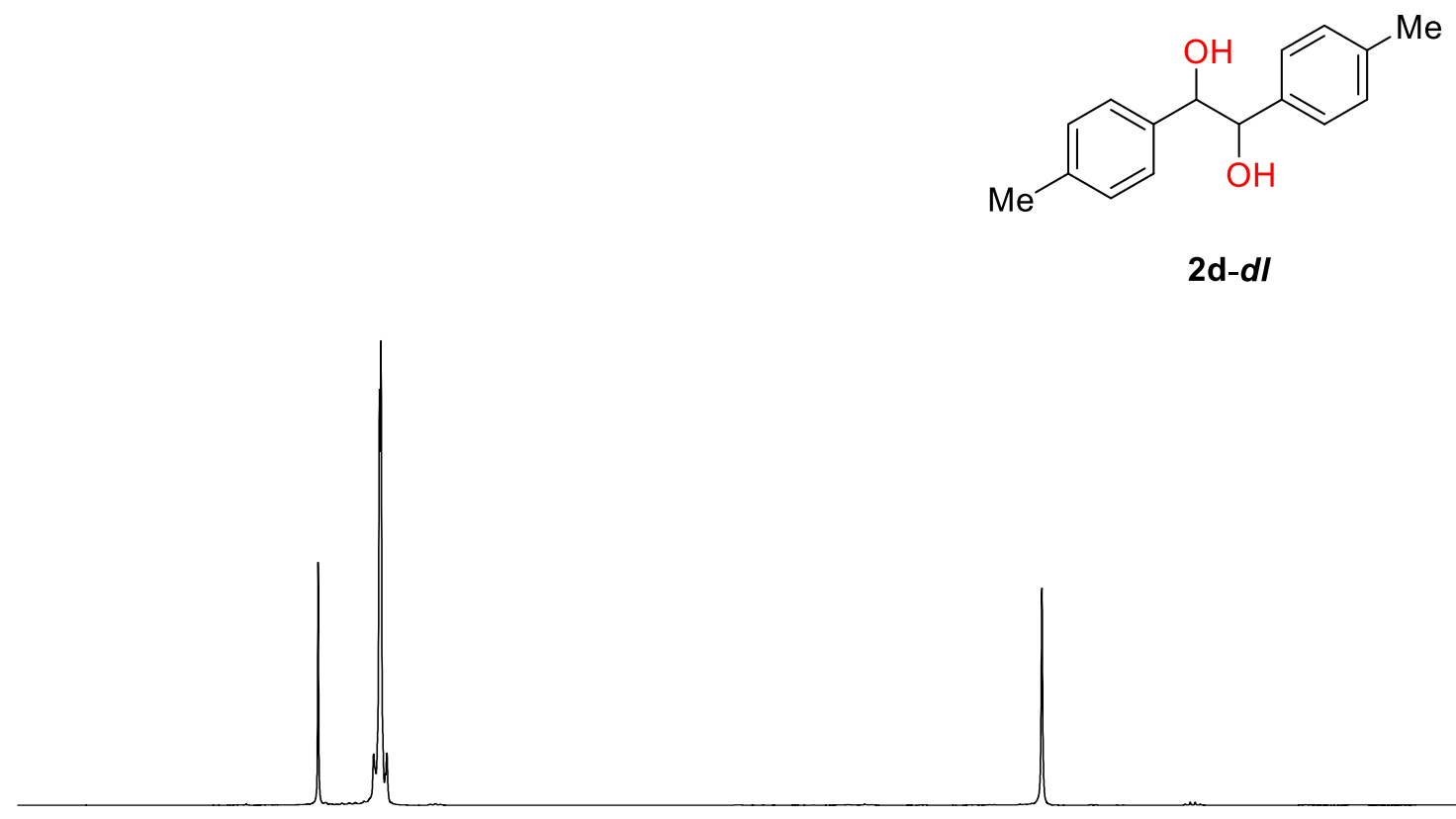

2d-dl
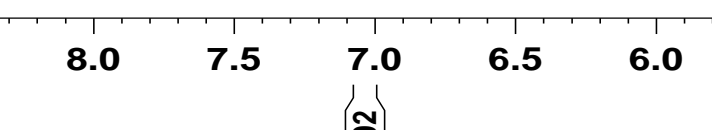

䟤

Figure S9. ${ }^{1} \mathrm{H}$ NMR spectra of $\mathbf{2 d}-\boldsymbol{d} \boldsymbol{l}\left(\mathrm{CDCl}_{3}, 400 \mathrm{M}\right)$. 


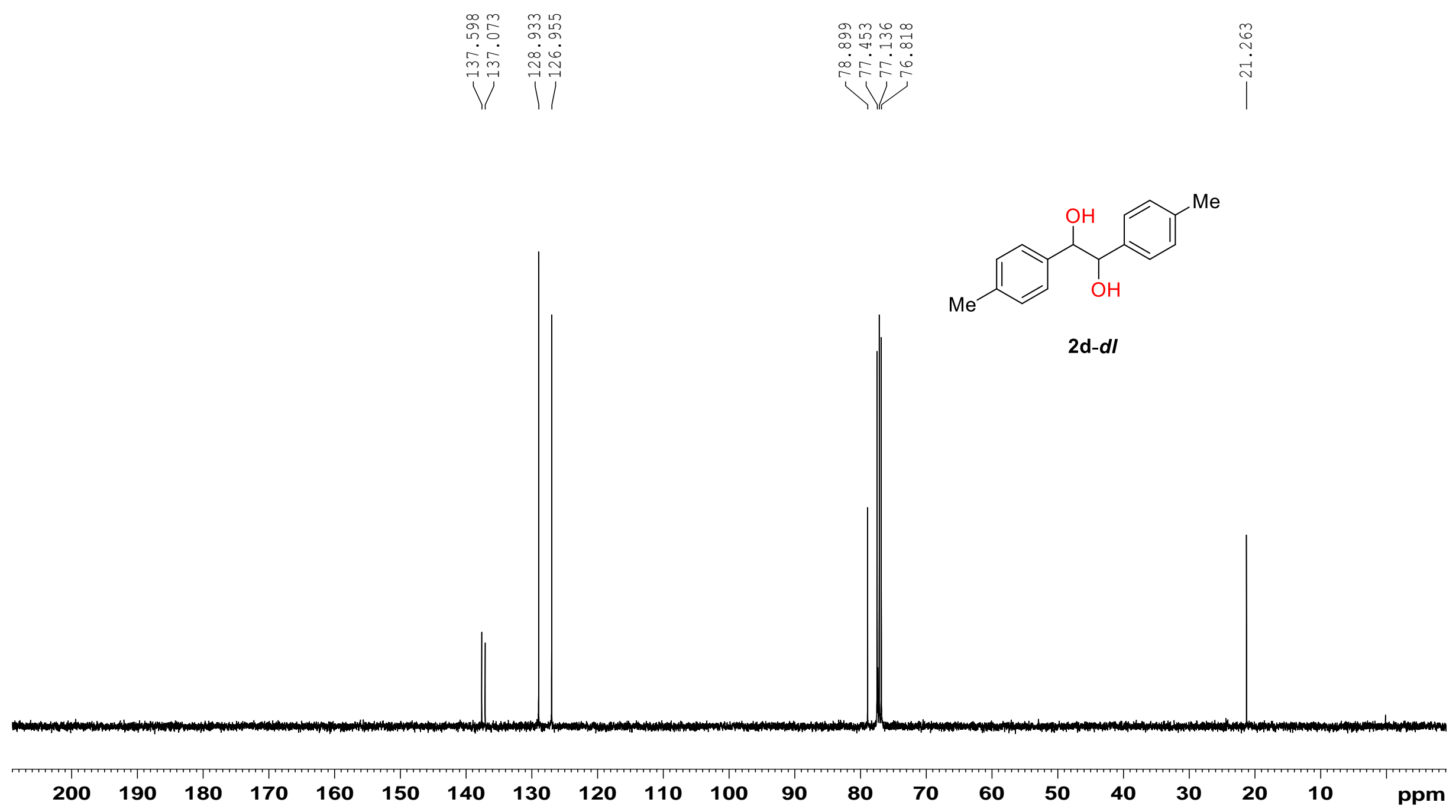

Figure S10. ${ }^{13} \mathrm{C}$ NMR spectra of $\mathbf{2 d}-\boldsymbol{d l}\left(\mathrm{CDCl}_{3}, 100 \mathrm{M}\right)$. 


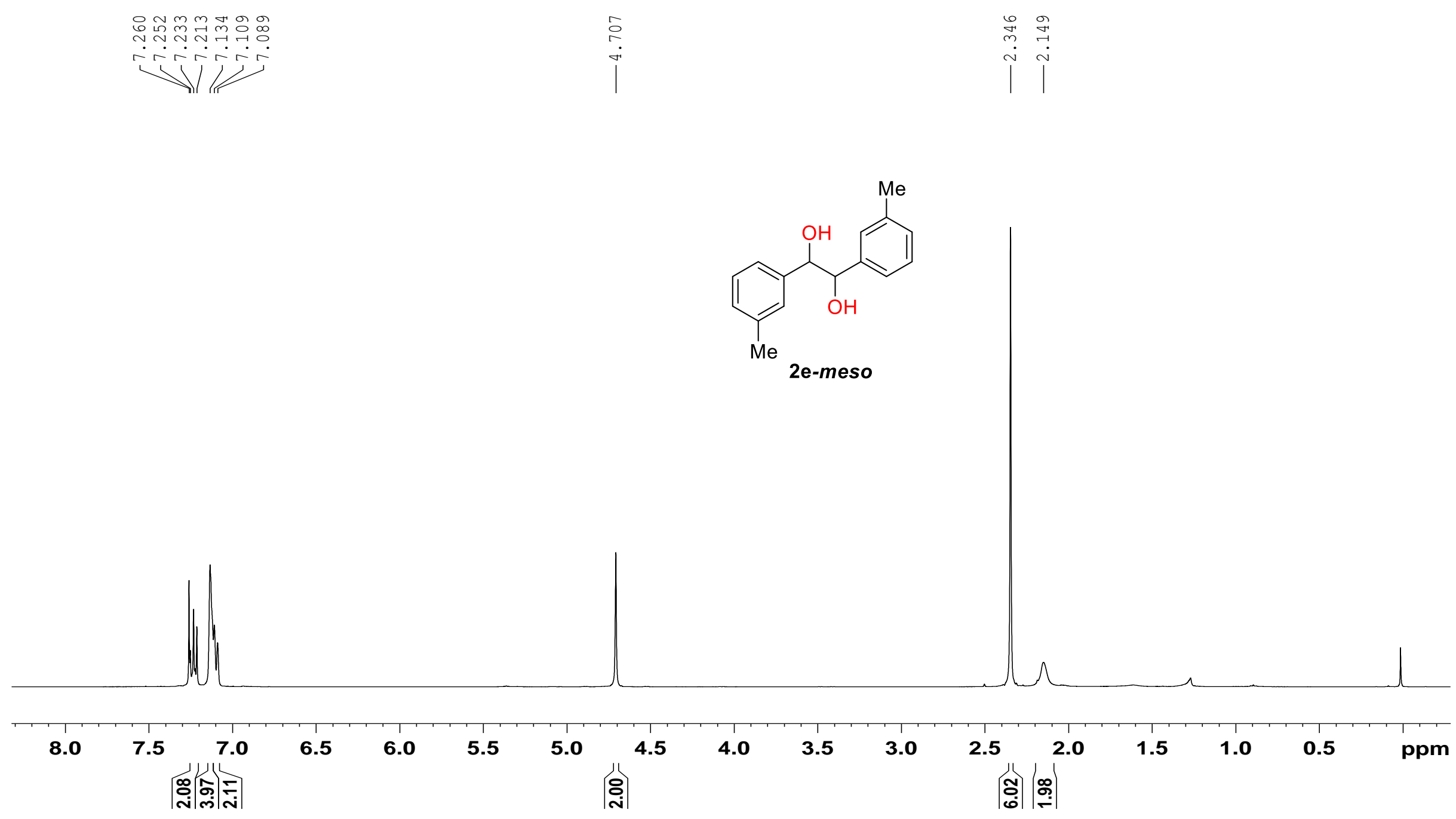

Figure S11. ${ }^{1} \mathrm{H}$ NMR spectra of 2e-meso $\left(\mathrm{CDCl}_{3}, 400 \mathrm{M}\right)$. 

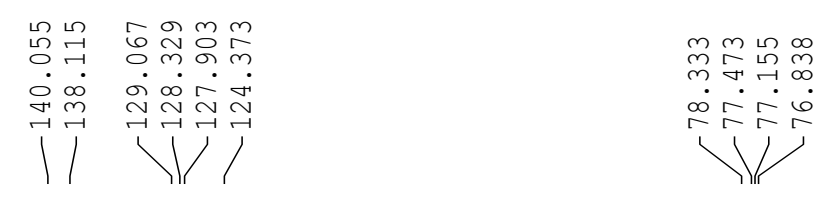



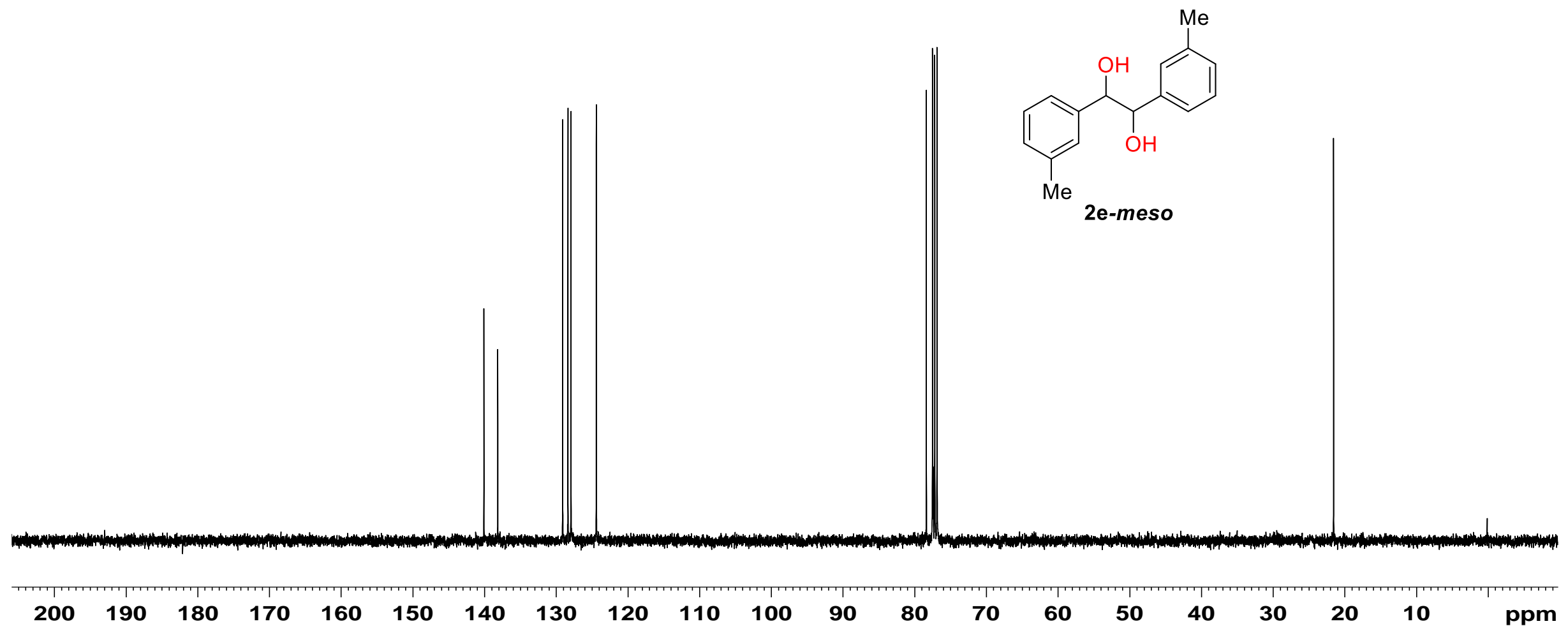

Figure S12. ${ }^{13} \mathrm{C}$ NMR spectra of $2 \mathrm{e}-$-meso $\left(\mathrm{CDCl}_{3}, 100 \mathrm{M}\right)$. 


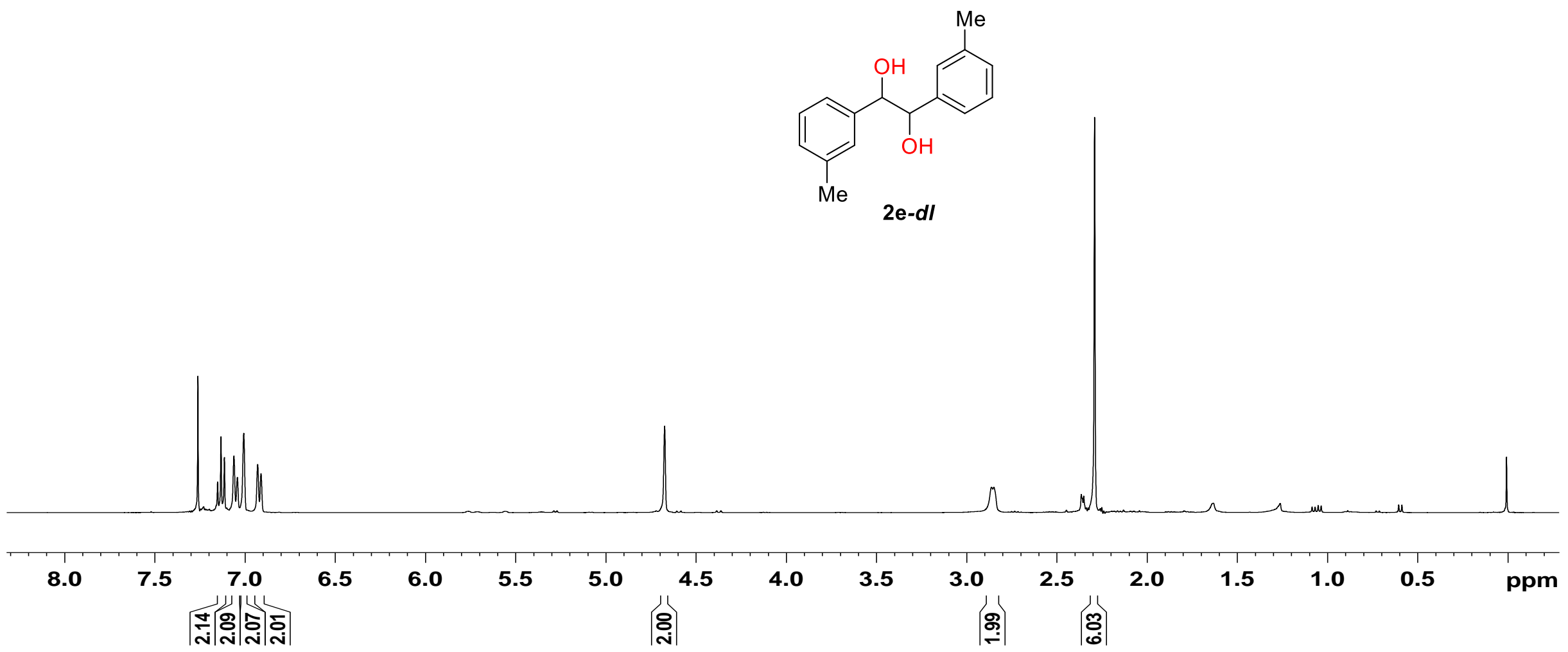

Figure S13. ${ }^{1} \mathrm{H}$ NMR spectra of $\mathbf{2 e}-\boldsymbol{d l}\left(\mathrm{CDCl}_{3}, 400 \mathrm{M}\right)$. 


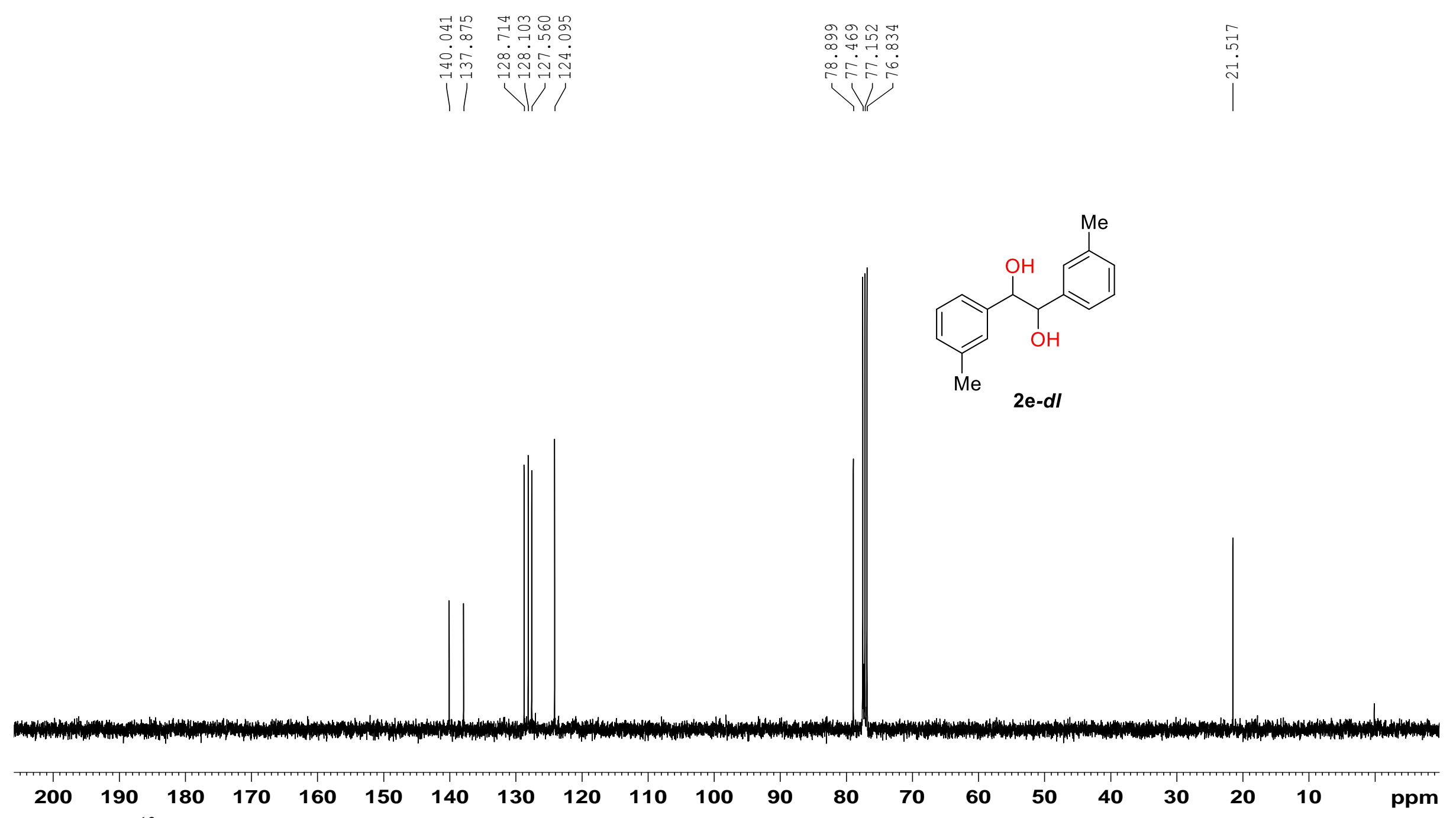

Figure S14. ${ }^{13} \mathrm{C}$ NMR spectra of $\mathbf{2 e}-\boldsymbol{d} \boldsymbol{l}\left(\mathrm{CDCl}_{3}, 100 \mathrm{M}\right)$. 


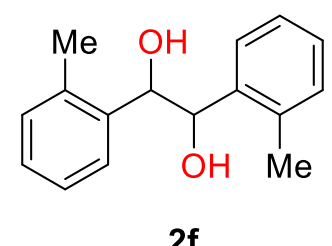

$2 f$

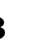

7

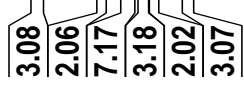

6

6

Figure S15. ${ }^{1} \mathrm{H}$ NMR spectra of $\mathbf{2} \mathbf{f}\left(\mathrm{CDCl}_{3}, 400 \mathrm{M}\right)$. 

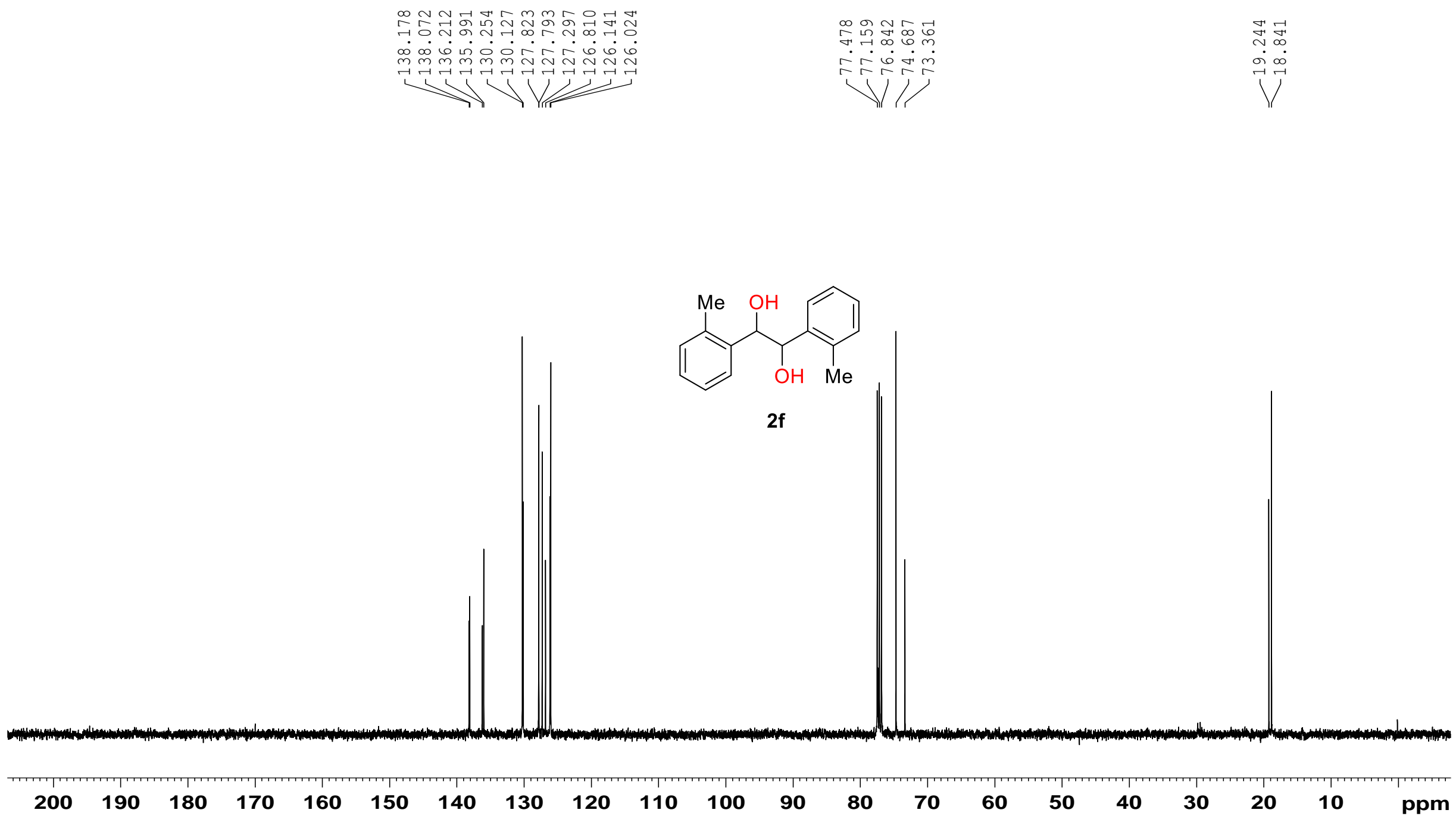

Figure S16. ${ }^{13} \mathrm{C}$ NMR spectra of $\mathbf{2 f}\left(\mathrm{CDCl}_{3}, 100 \mathrm{M}\right)$. 


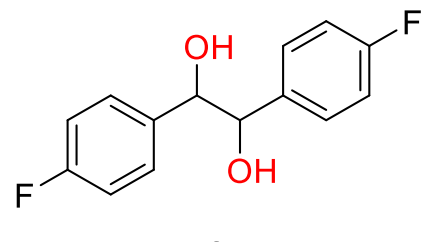

$2 \mathrm{~g}$

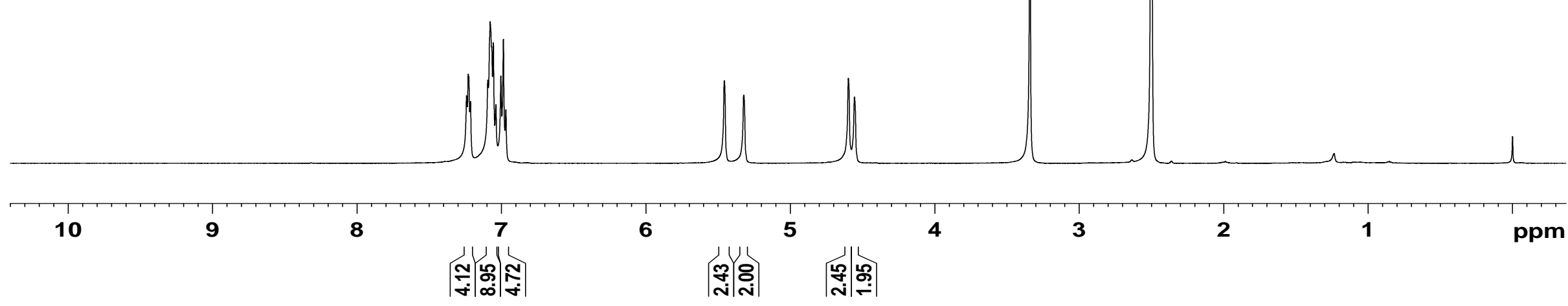

Figure S17. ${ }^{1} \mathrm{H}$ NMR spectra of $\mathbf{2 g}\left(d^{6}\right.$-DMSO, 500M). 

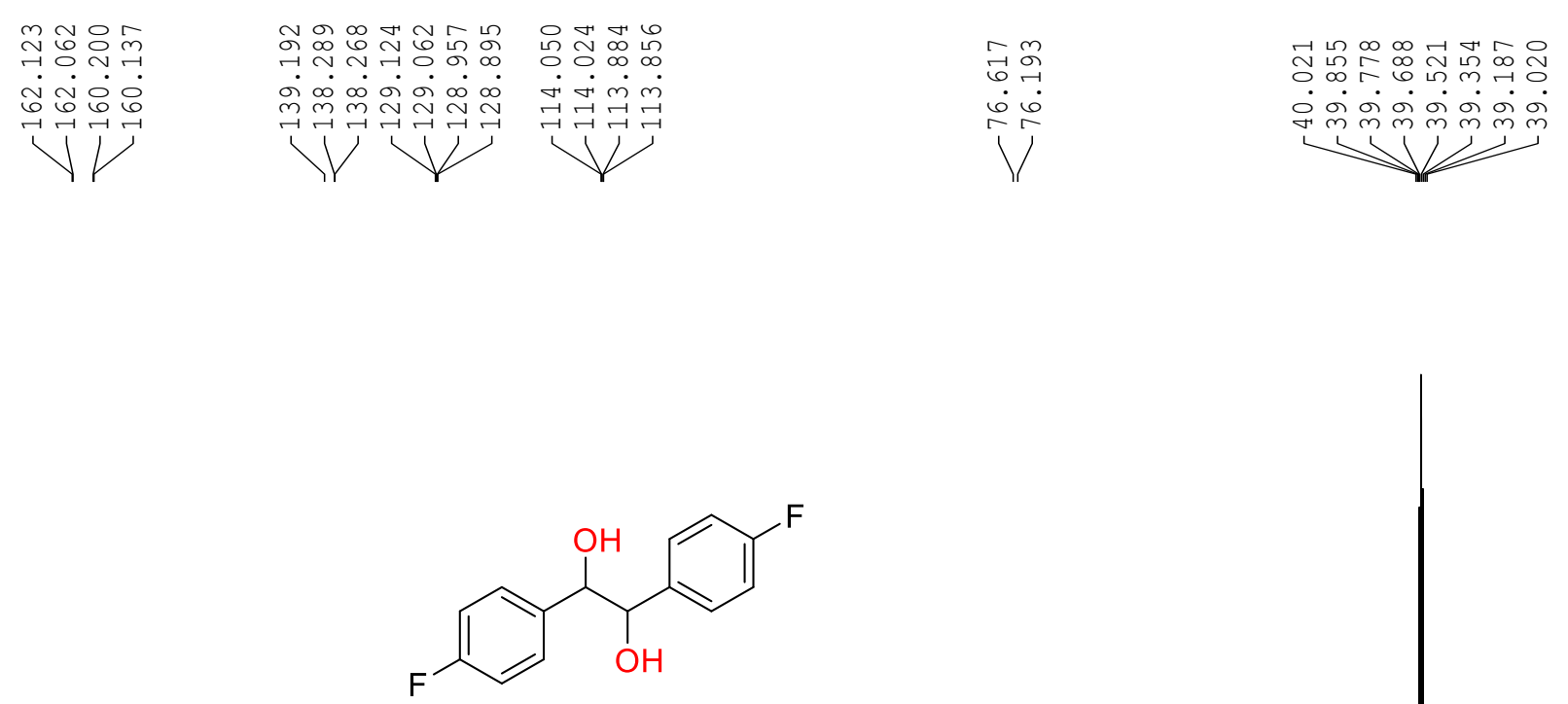

$2 \mathrm{~g}$

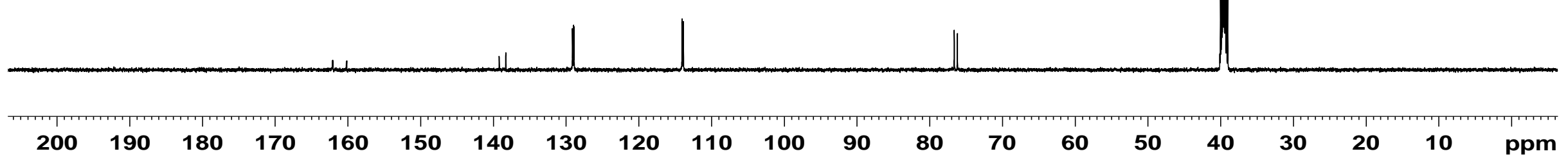

Figure S18. ${ }^{13} \mathrm{C}$ NMR spectra of $\mathbf{2 g}\left(d^{6}\right.$-DMSO, $\left.125 \mathrm{M}\right)$. 


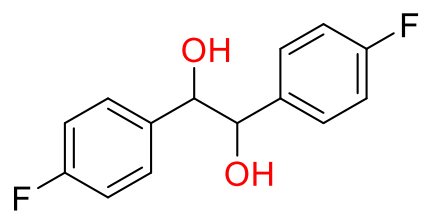

$2 \mathrm{~g}$

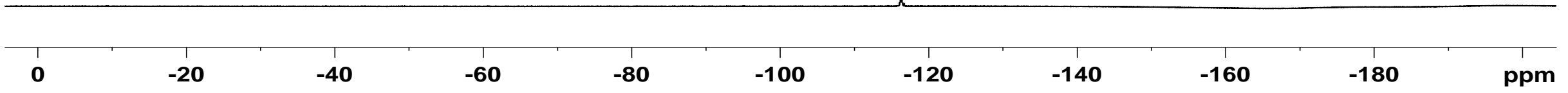

Figure S19. ${ }^{19} \mathrm{~F}$ NMR spectra of $\mathbf{2 g}\left(d^{6}\right.$-DMSO, 471M). 


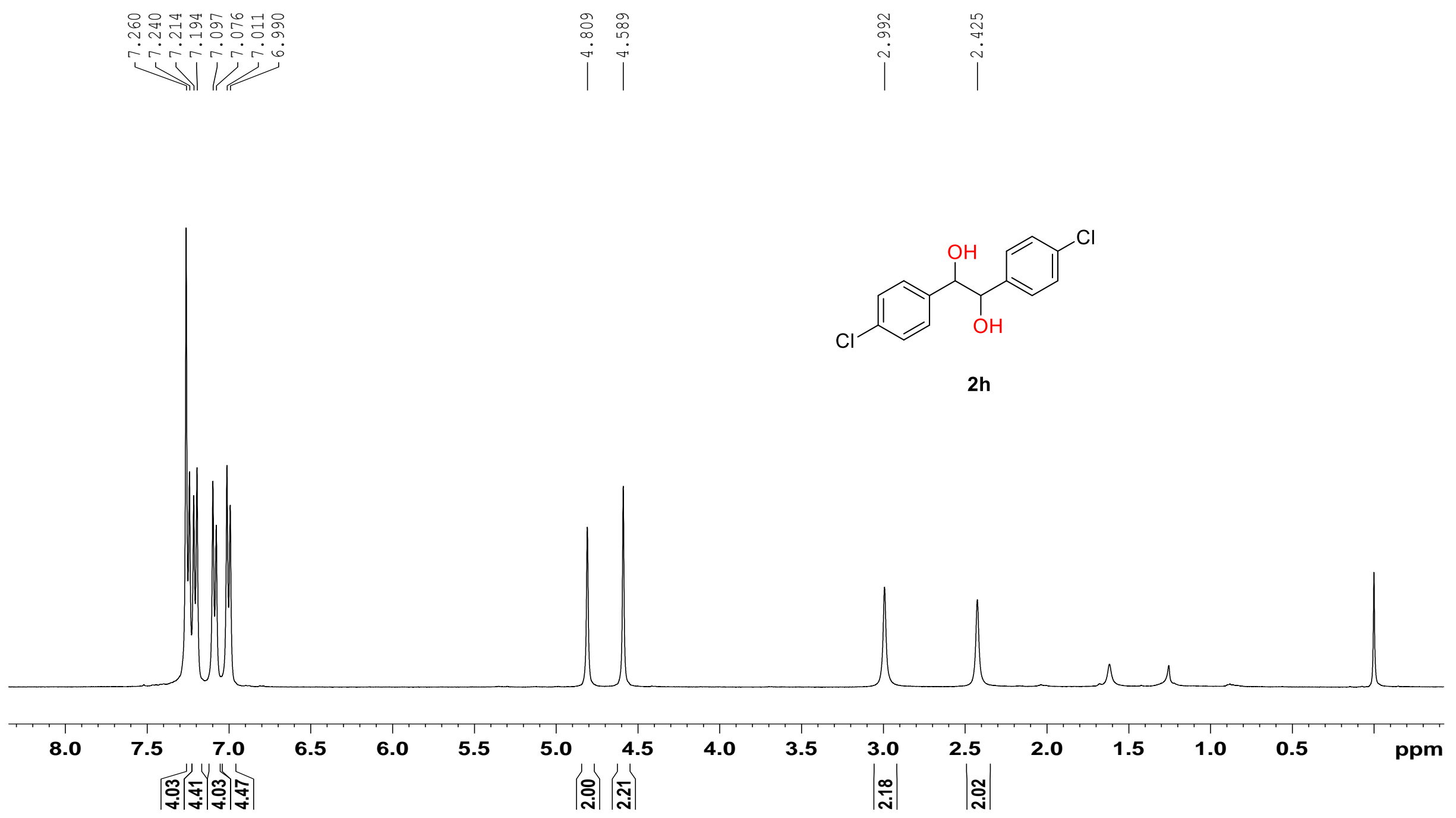

Figure S20. ${ }^{1} \mathrm{H}$ NMR spectra of $\mathbf{2 h}\left(\mathrm{CDCl}_{3}, 400 \mathrm{M}\right)$. 

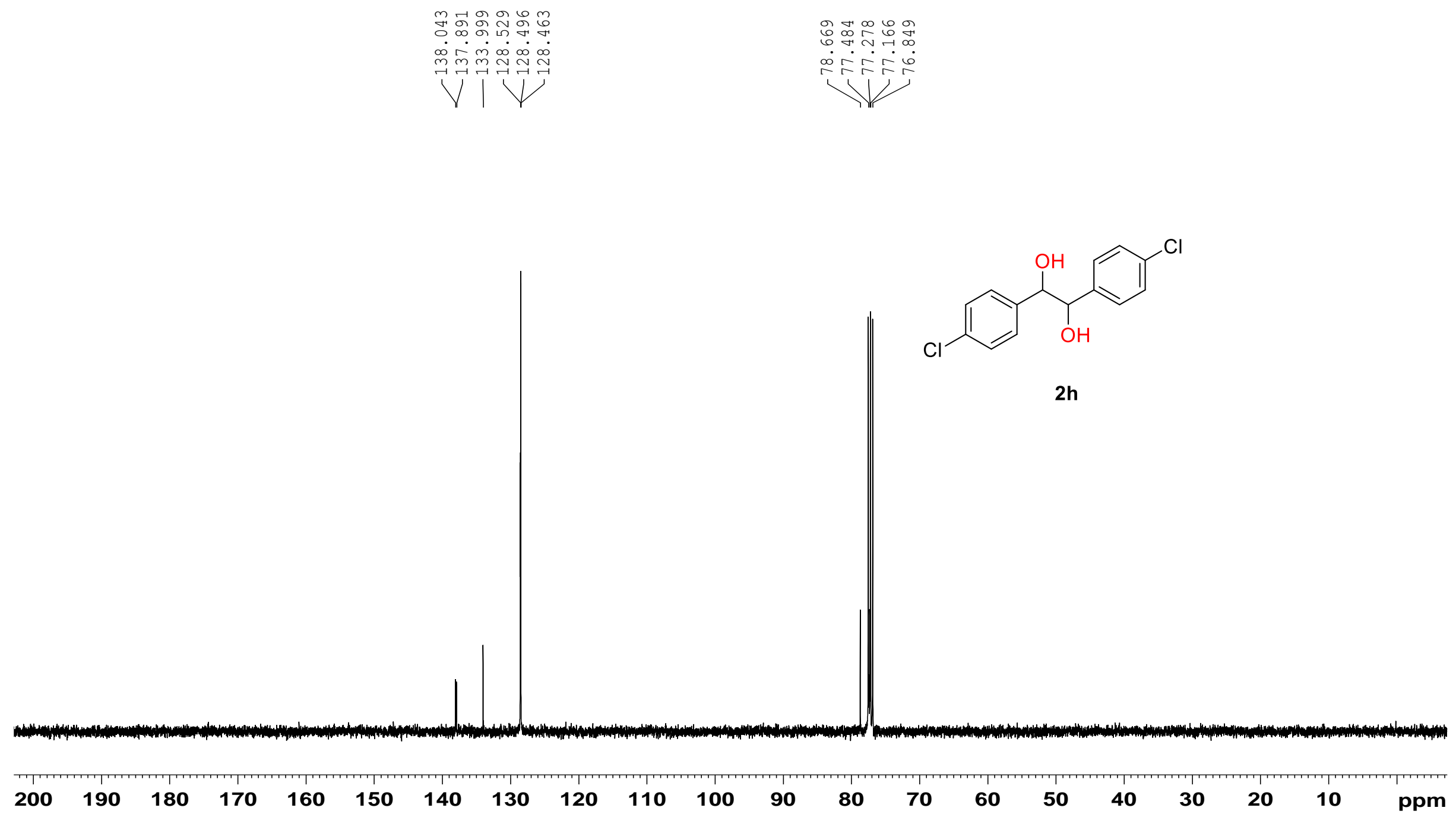

Figure S21. ${ }^{13} \mathrm{C}$ NMR spectra of $\mathbf{2 h}\left(\mathrm{CDCl}_{3}, 100 \mathrm{M}\right)$. 


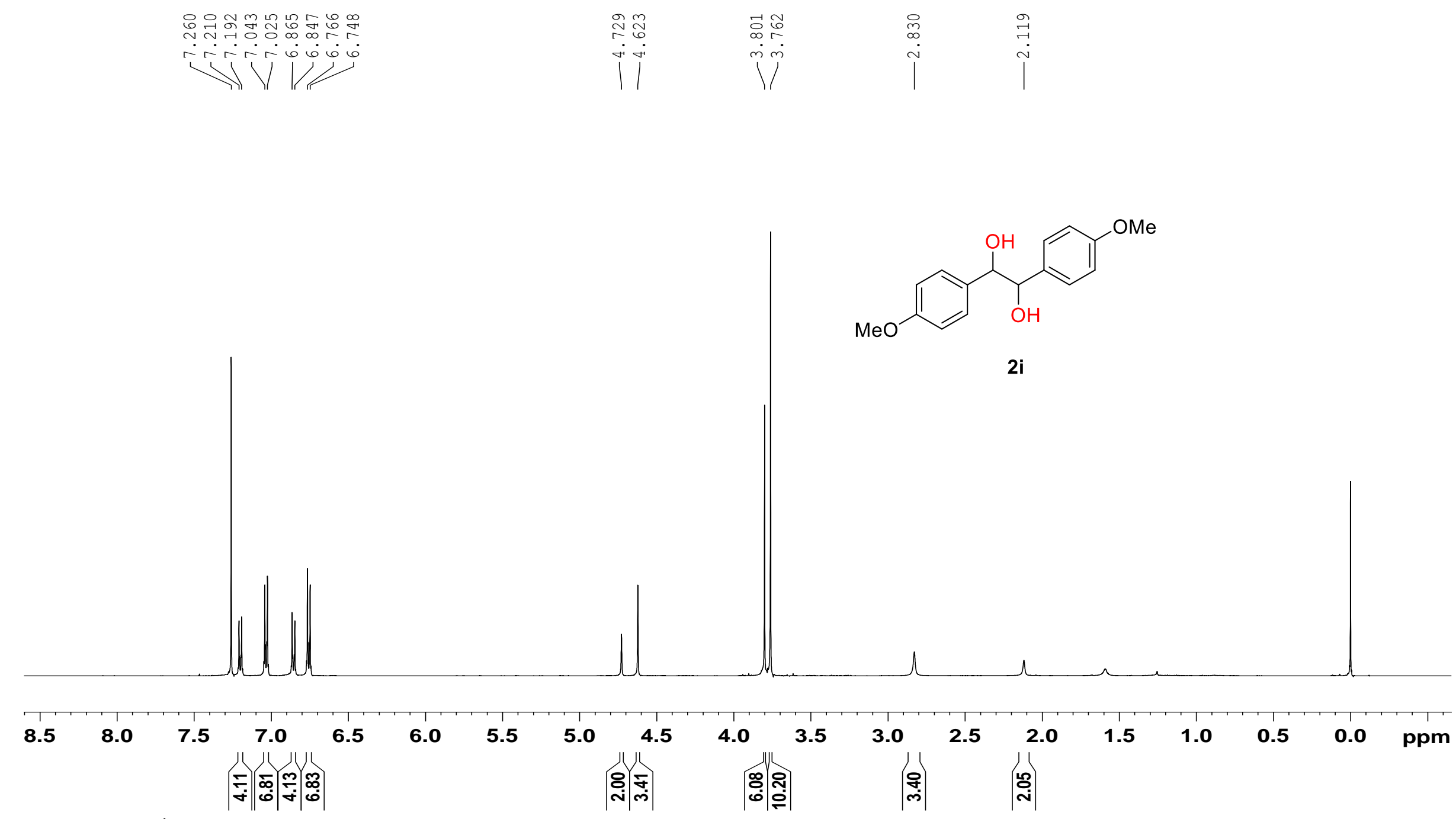

Figure S22. ${ }^{1} \mathrm{H}$ NMR spectra of $\mathbf{2 i}\left(\mathrm{CDCl}_{3}, 500 \mathrm{M}\right)$. 


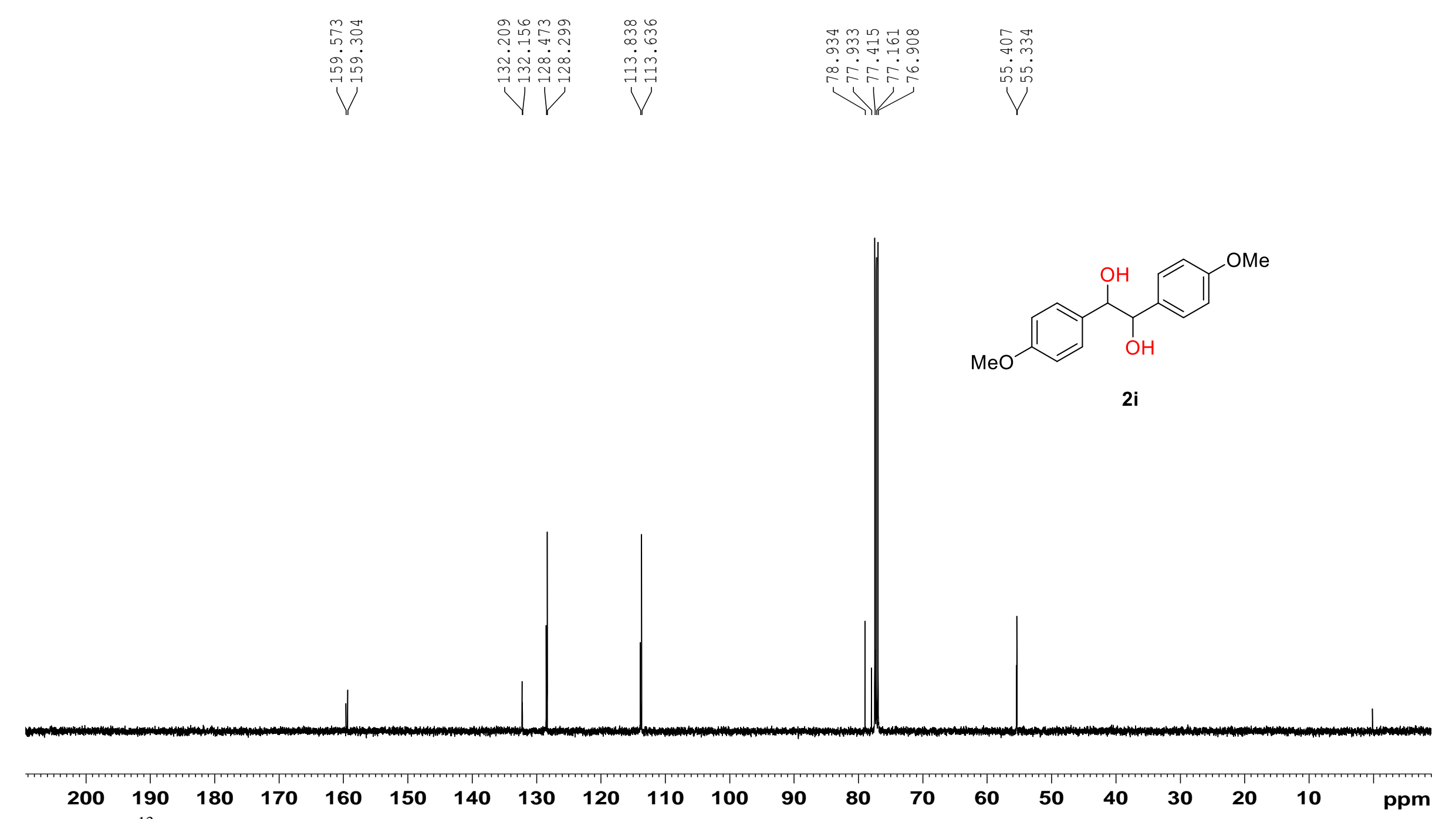

Figure S23. ${ }^{13} \mathrm{C}$ NMR spectra of $\mathbf{2 i}\left(\mathrm{CDCl}_{3}, 125 \mathrm{M}\right)$. 


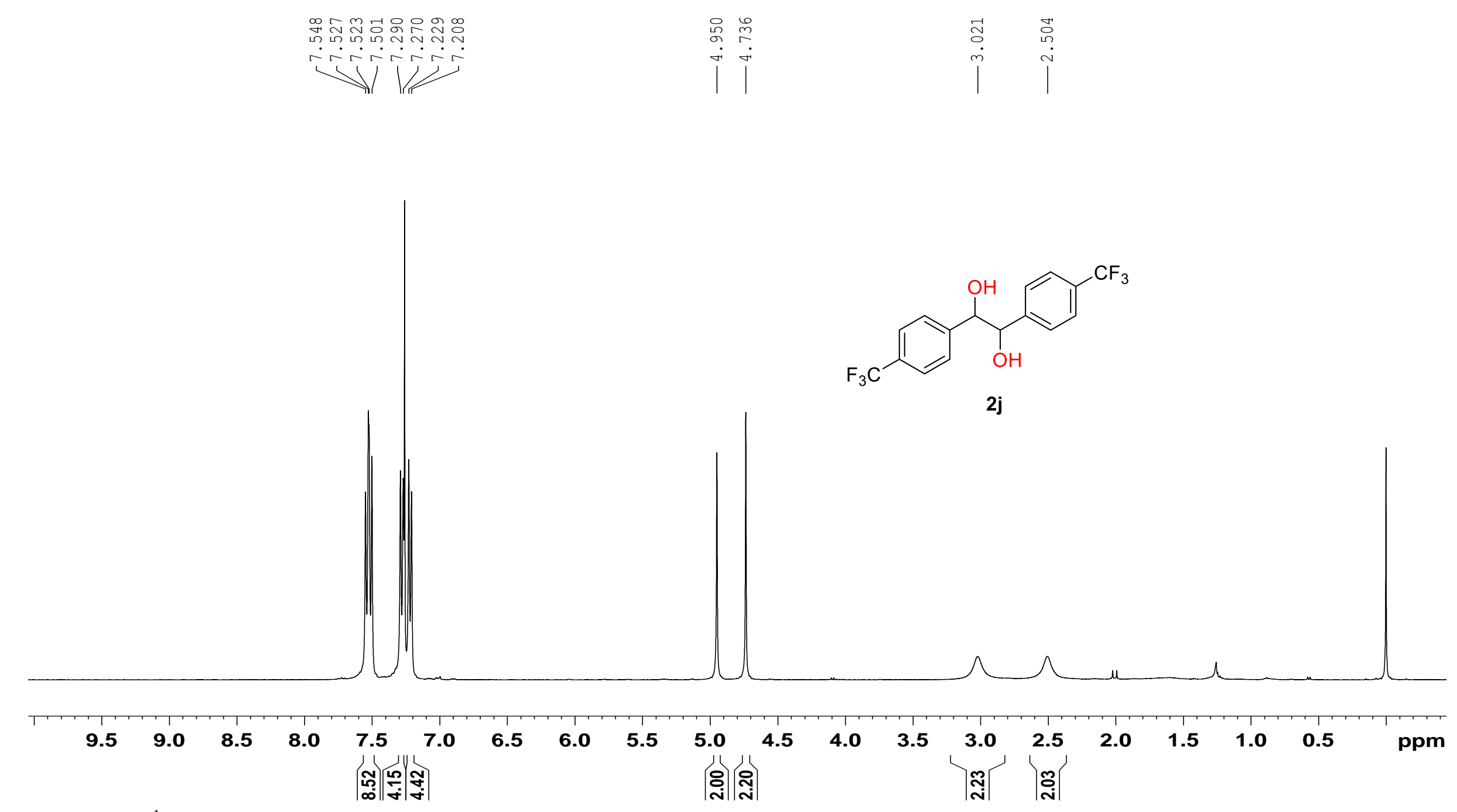

Figure S24. ${ }^{1} \mathrm{H}$ NMR spectra of $\mathbf{2} \mathbf{j}\left(\mathrm{CDCl}_{3}, 400 \mathrm{M}\right)$. 

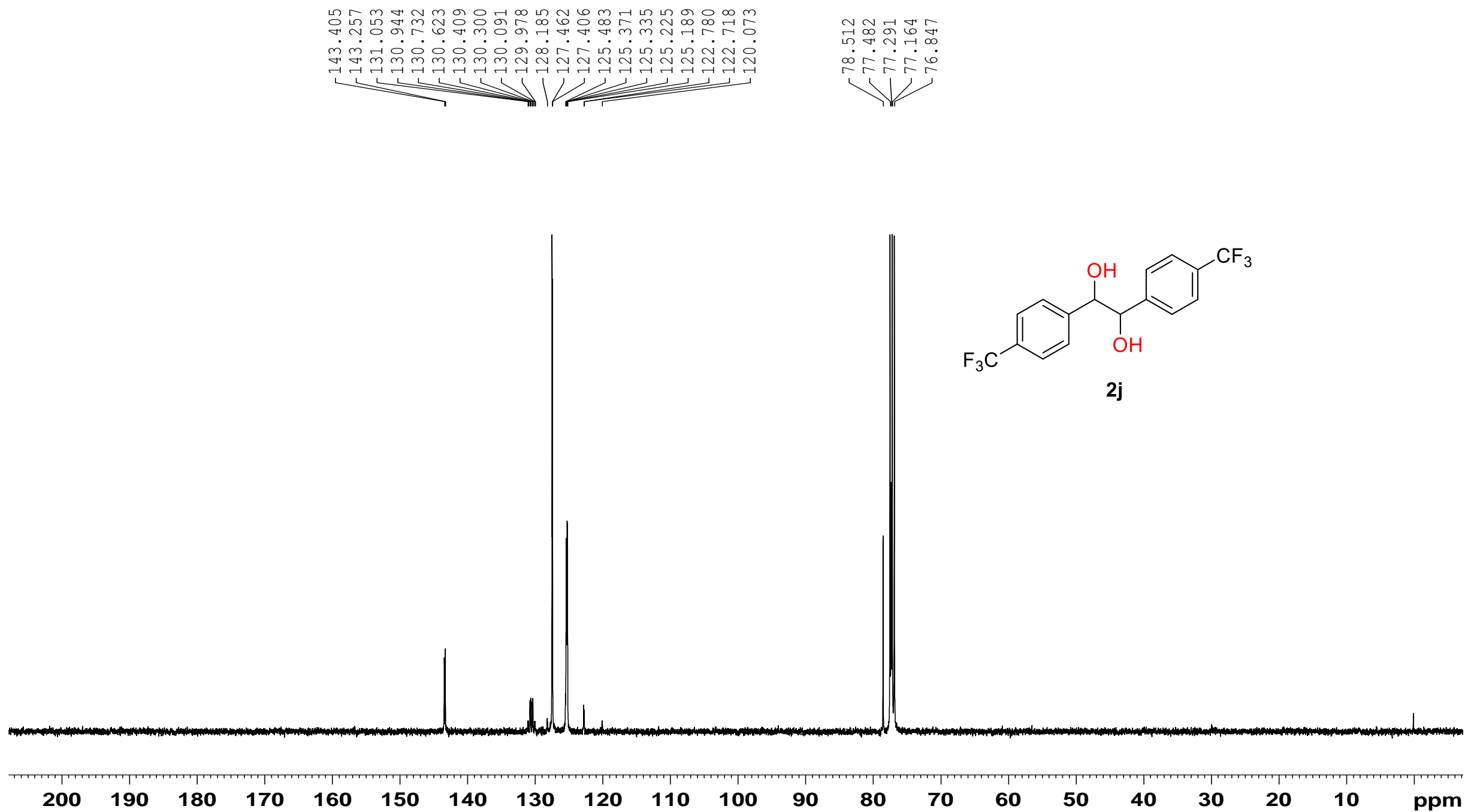

Figure S25. ${ }^{13} \mathrm{C}$ NMR spectra of $\mathbf{2} \mathbf{j}\left(\mathrm{CDCl}_{3}, 100 \mathrm{M}\right)$. 


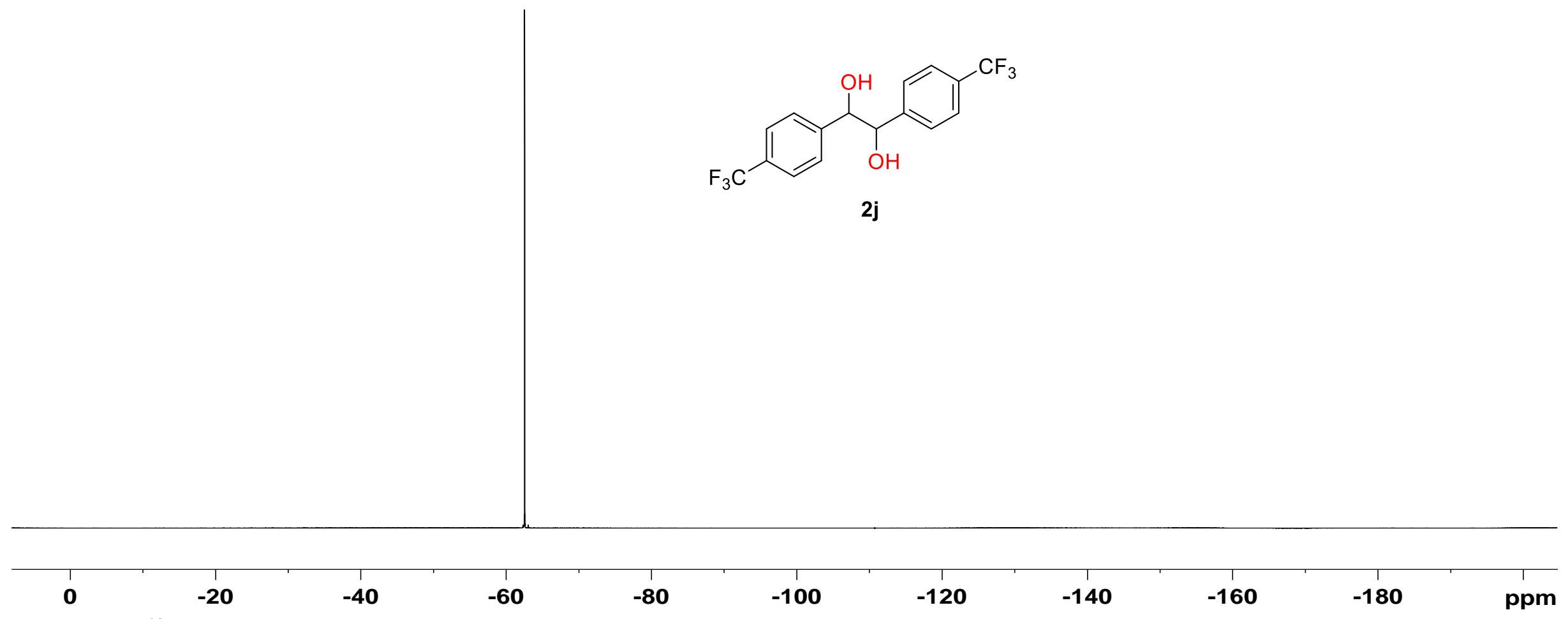

Figure S26. ${ }^{19} \mathrm{~F}$ NMR spectra of $\mathbf{2 j}\left(\mathrm{CDCl}_{3}, 376 \mathrm{M}\right)$. 


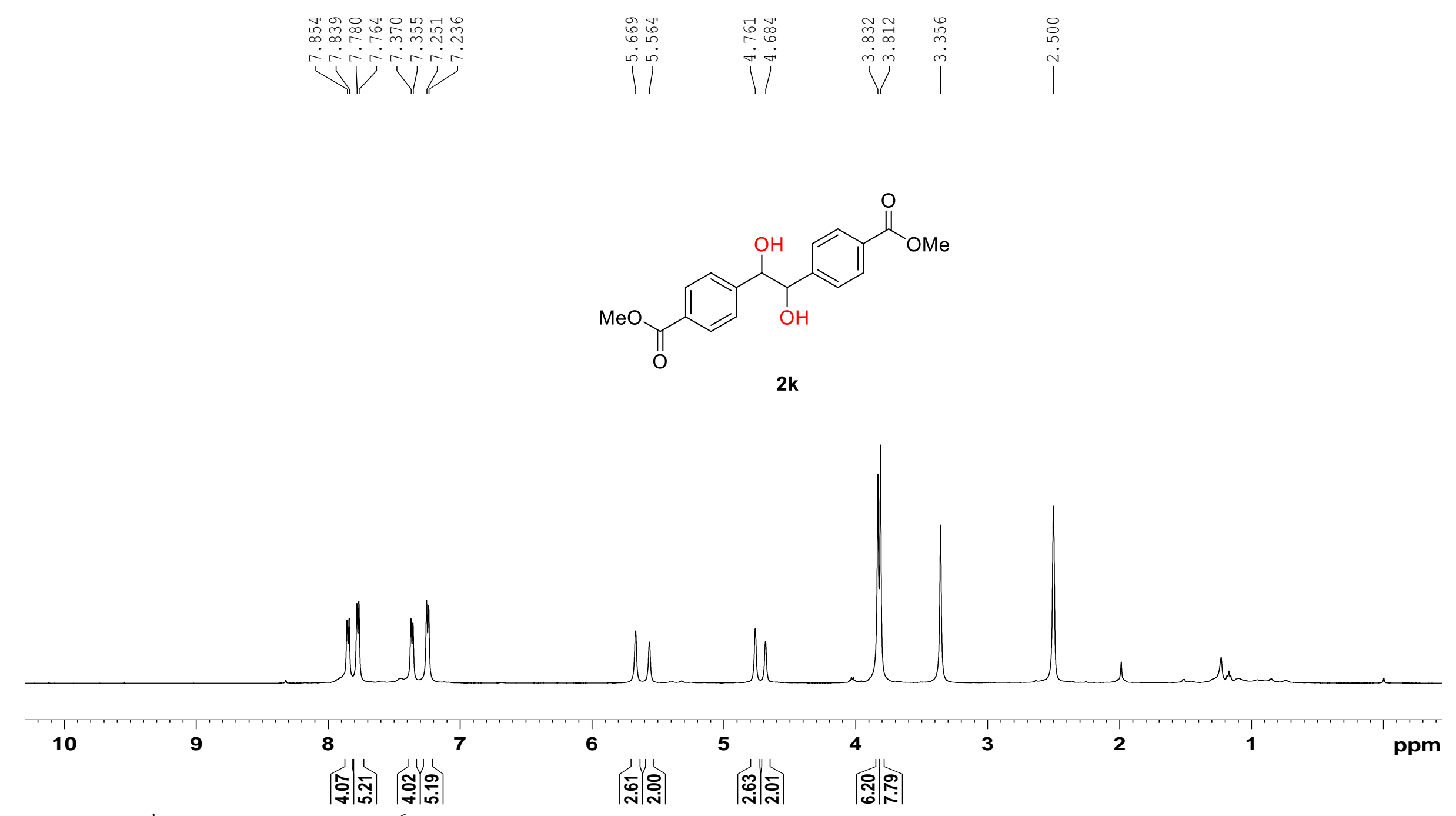

Figure S27. ${ }^{1} \mathrm{H}$ NMR spectra of $\mathbf{2 k}\left(d^{6}\right.$-DMSO, 500M). 

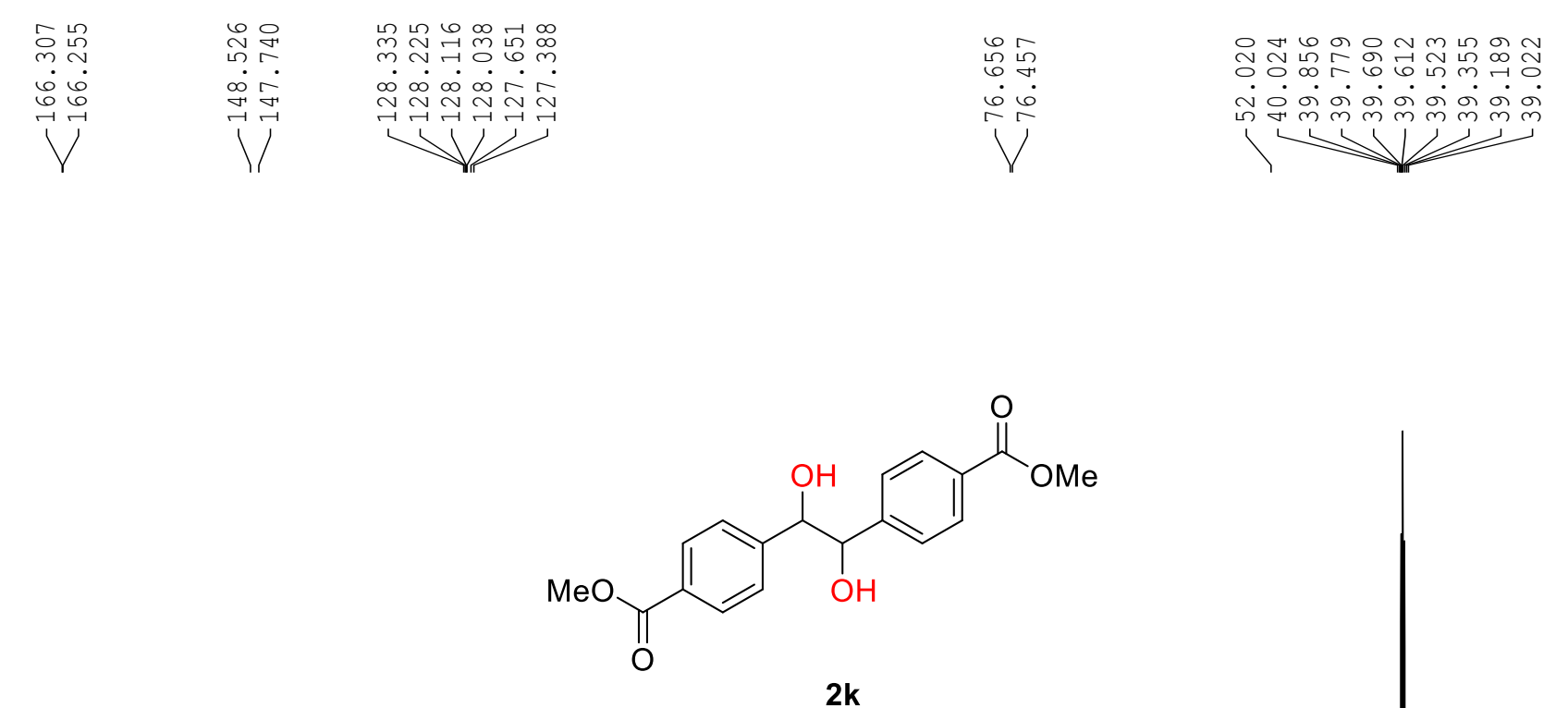

$2 k$

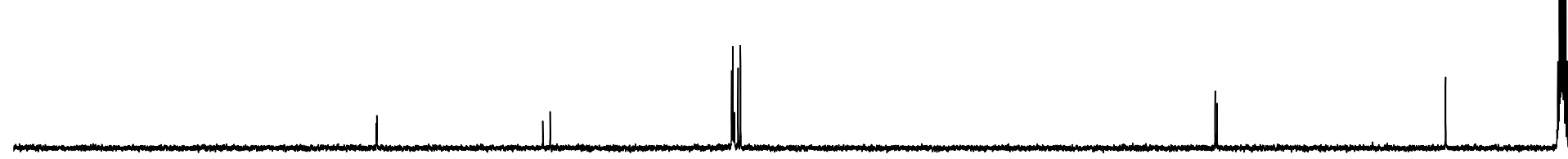

Figure S28. ${ }^{13} \mathrm{C}$ NMR spectra of $\mathbf{2 k}\left(d^{6}\right.$-DMSO, 125M). 

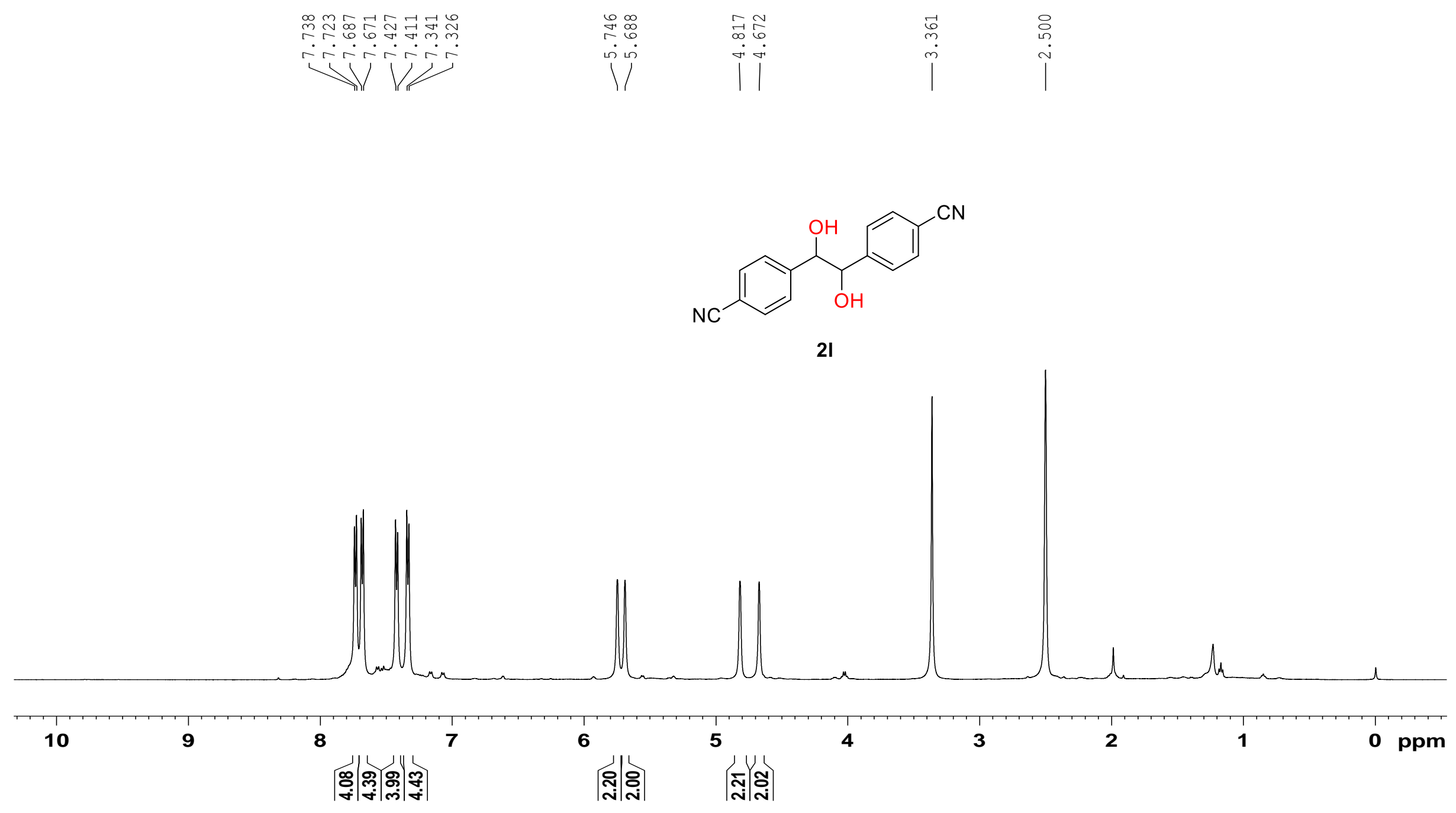

Figure S29. ${ }^{1} \mathrm{H}$ NMR spectra of $\mathbf{2 l}\left(d^{6}\right.$-DMSO, 500M). 

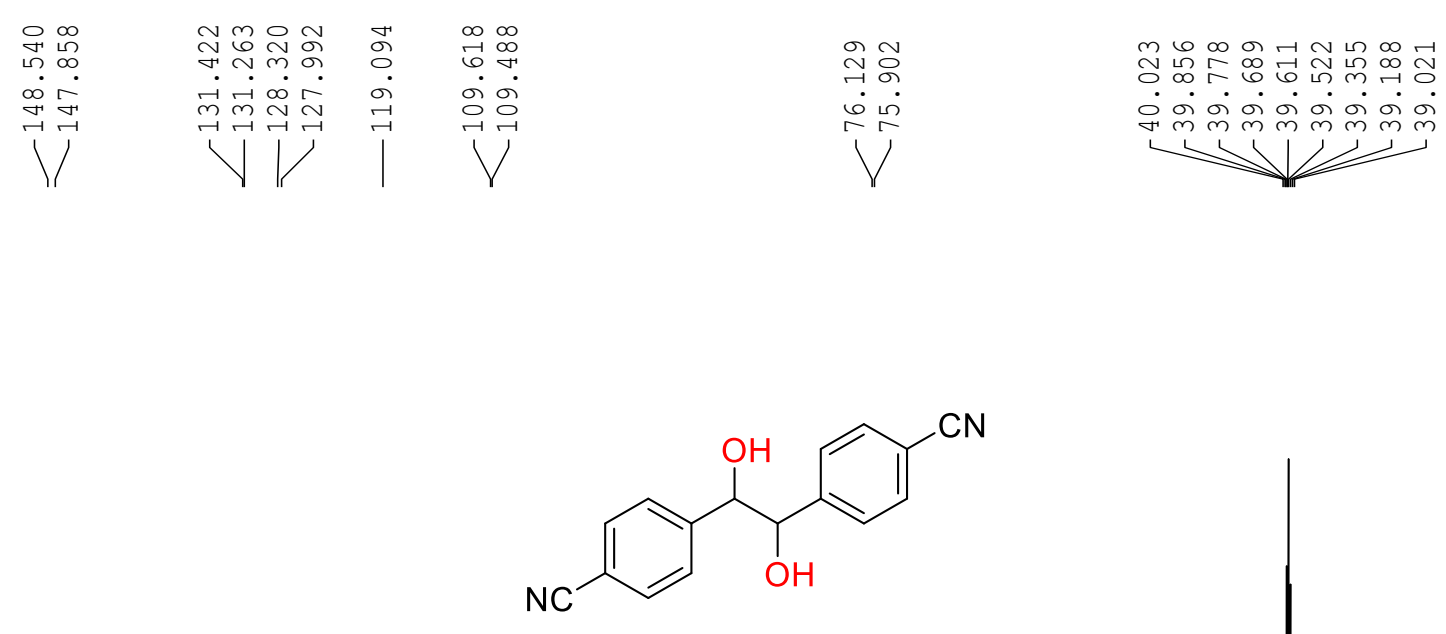

2I

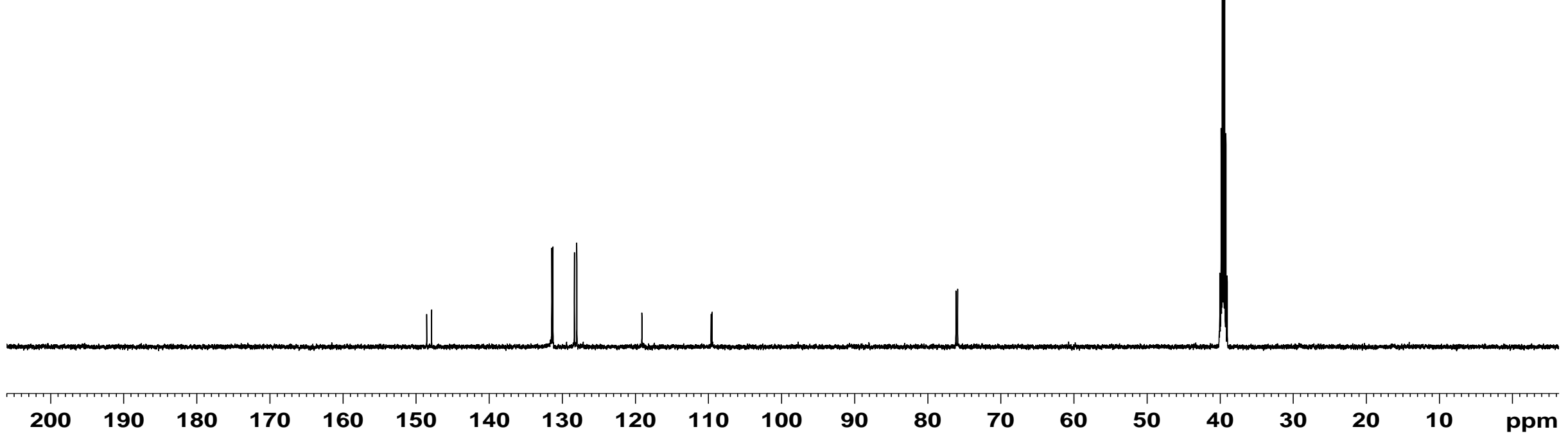

Figure S30. ${ }^{13} \mathrm{C}$ NMR spectra of $\mathbf{2 l}\left(d^{6}\right.$-DMSO, 125M). 


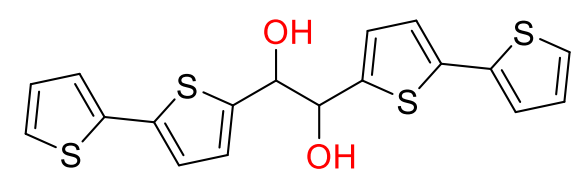

$2 m$

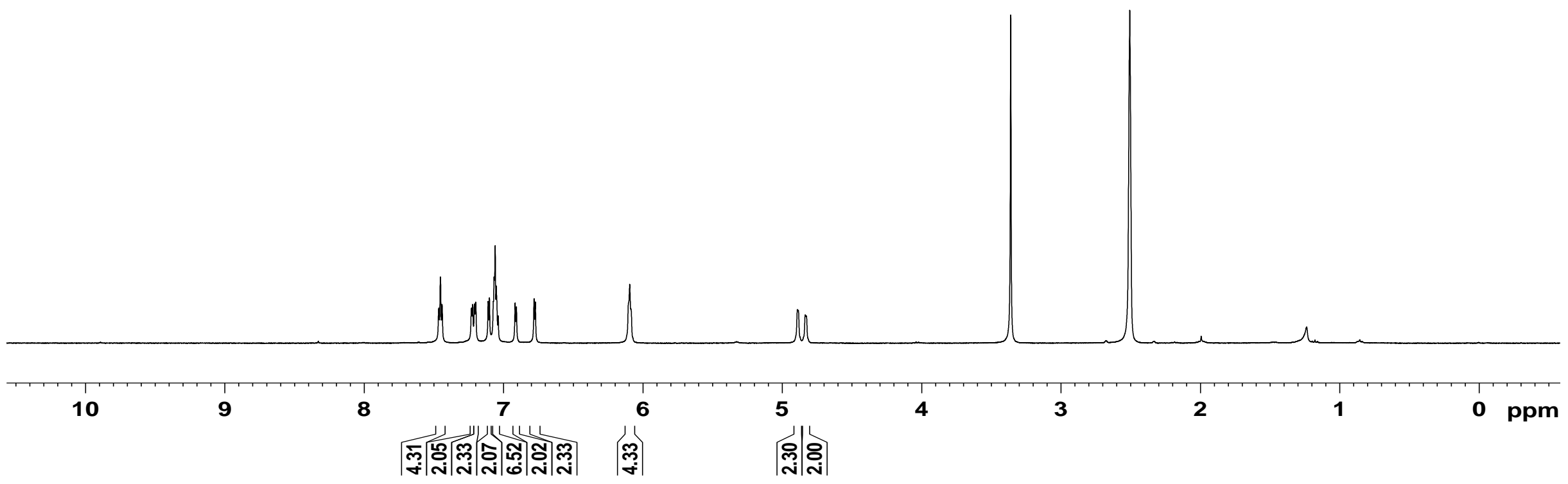

Figure S31. ${ }^{1} \mathrm{H}$ NMR spectra of $\mathbf{2 m}\left(d^{6}\right.$-DMSO, 400M). 

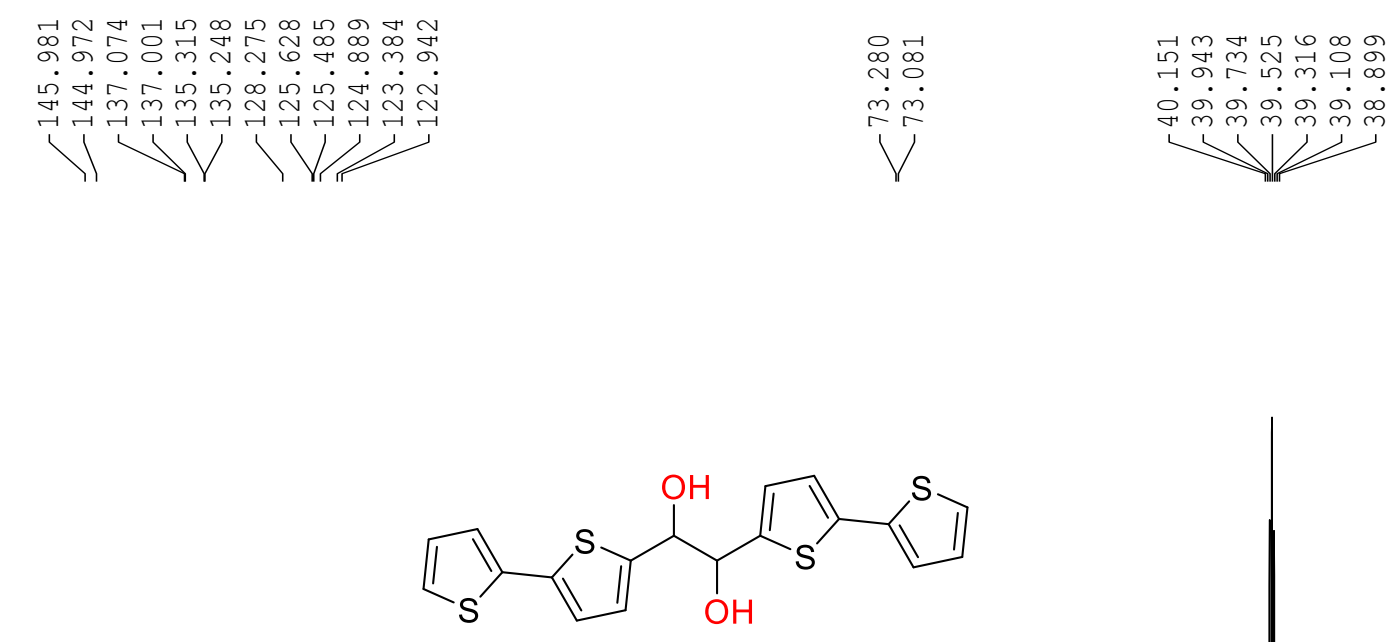

$2 m$

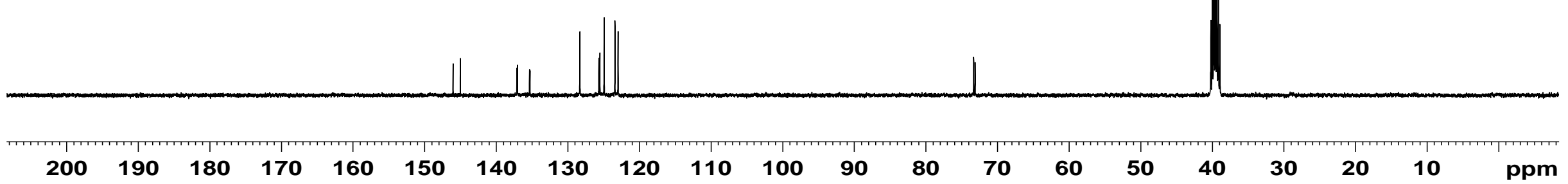

Figure S32. ${ }^{13} \mathrm{C}$ NMR spectra of $\mathbf{2 m}\left(d^{6}\right.$-DMSO, 100M). 


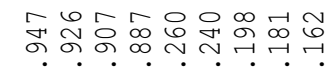

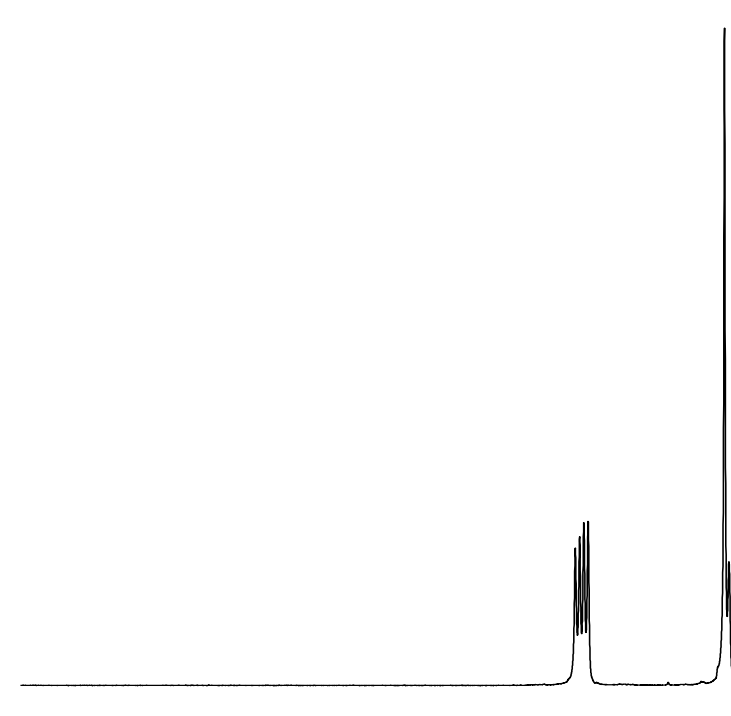

10

9

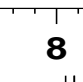

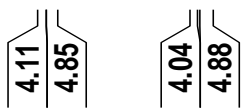

Figure S33. ${ }^{1} \mathrm{H}$ NMR spectra of $\mathbf{2 n}\left(\mathrm{CDCl}_{3}, 400 \mathrm{M}\right)$.

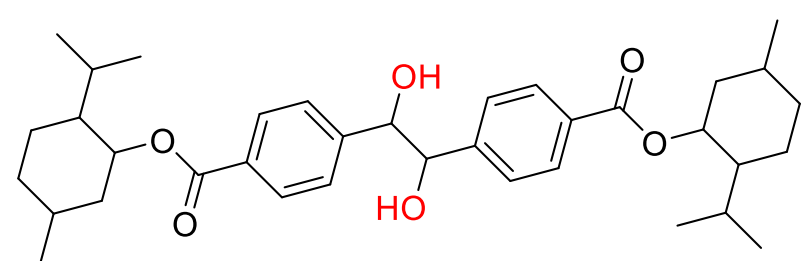

$2 n$

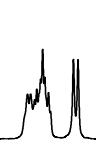

$\left|\begin{array}{l}0 \\ \mathfrak{o} \\ 0\end{array}\right|$

4

)

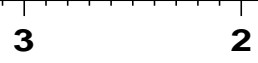

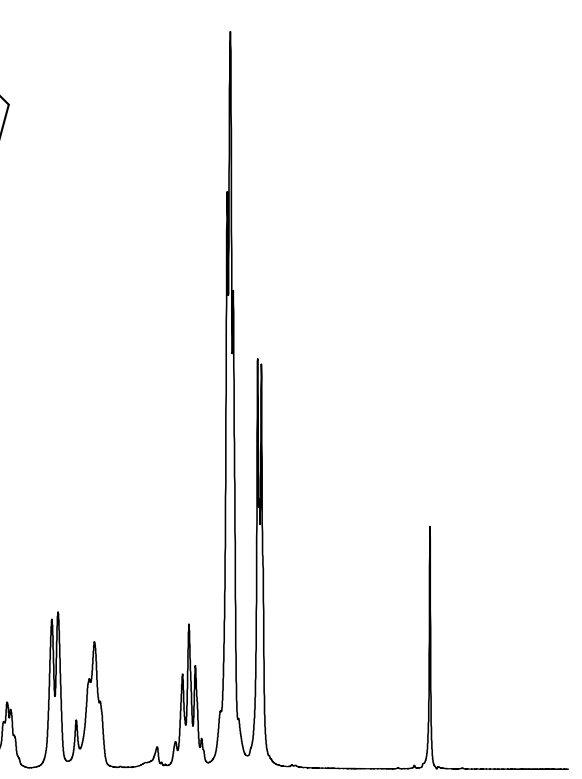

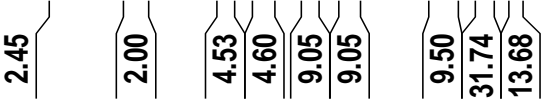
o ppm 

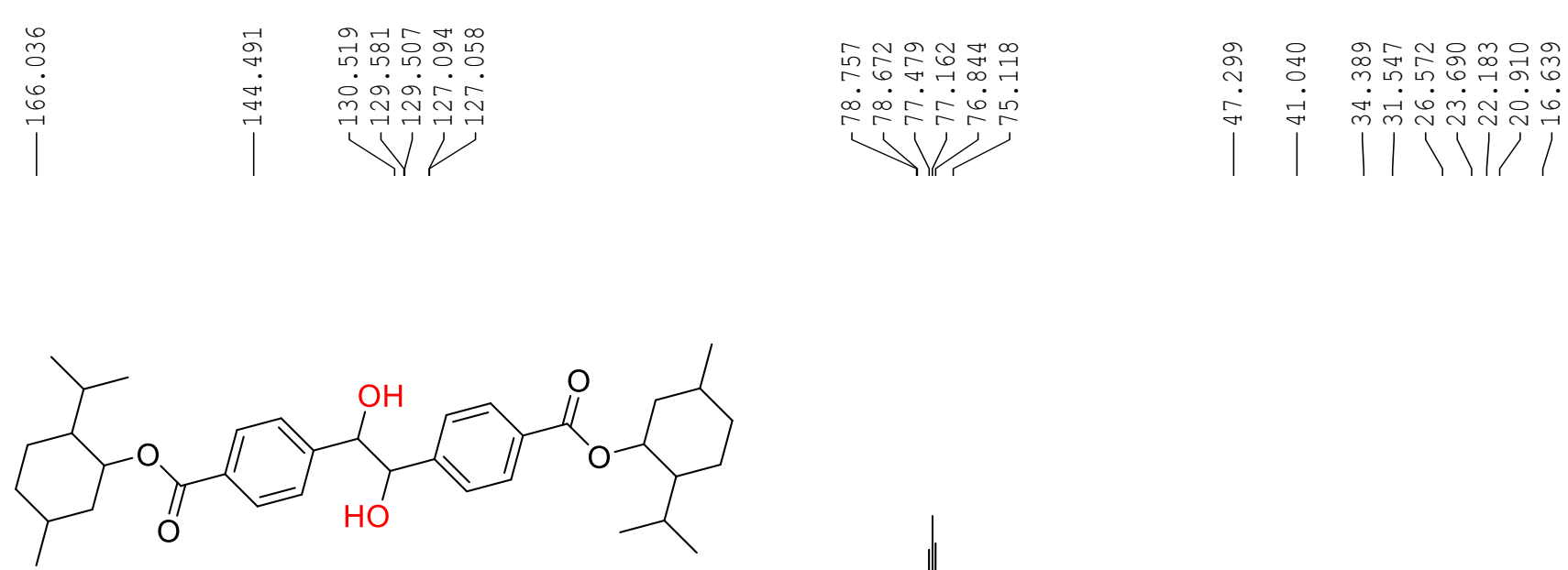

2n

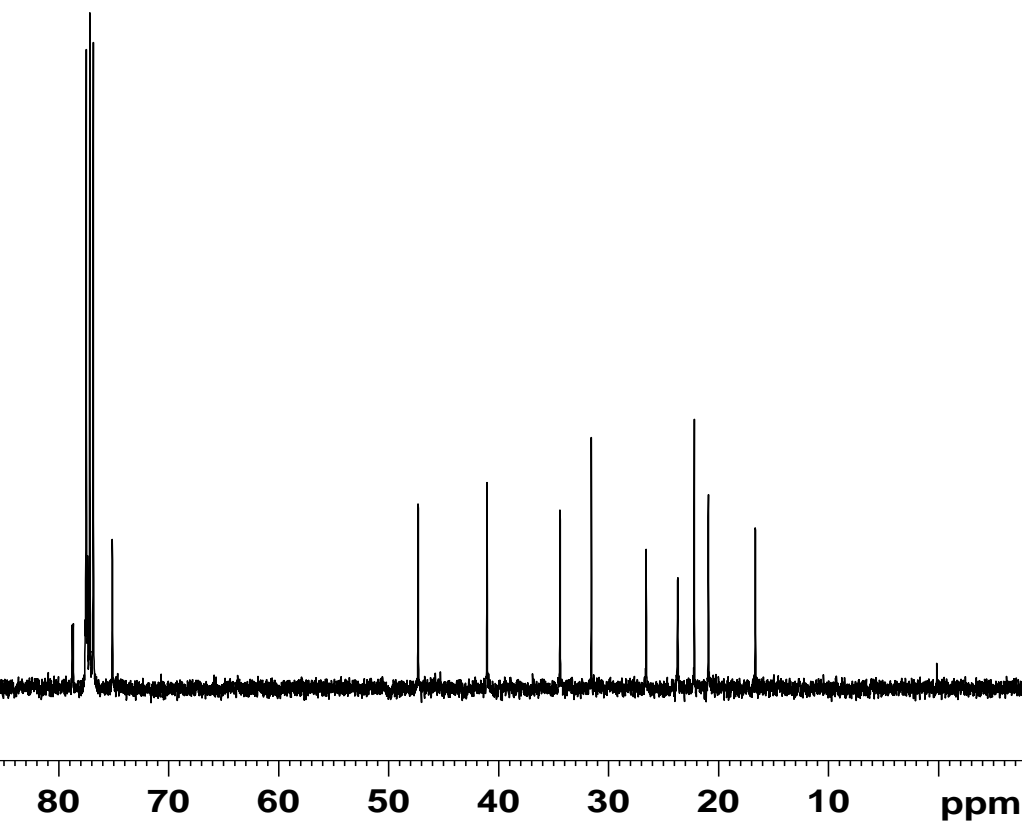
$\begin{array}{llllllll}200 & 190 & 180 & 170 & 160 & 150 & 140 & 130\end{array}$

Figure S34. ${ }^{13} \mathrm{C}$ NMR spectra of $\mathbf{2 n}\left(\mathrm{CDCl}_{3}, 100 \mathrm{M}\right)$. 


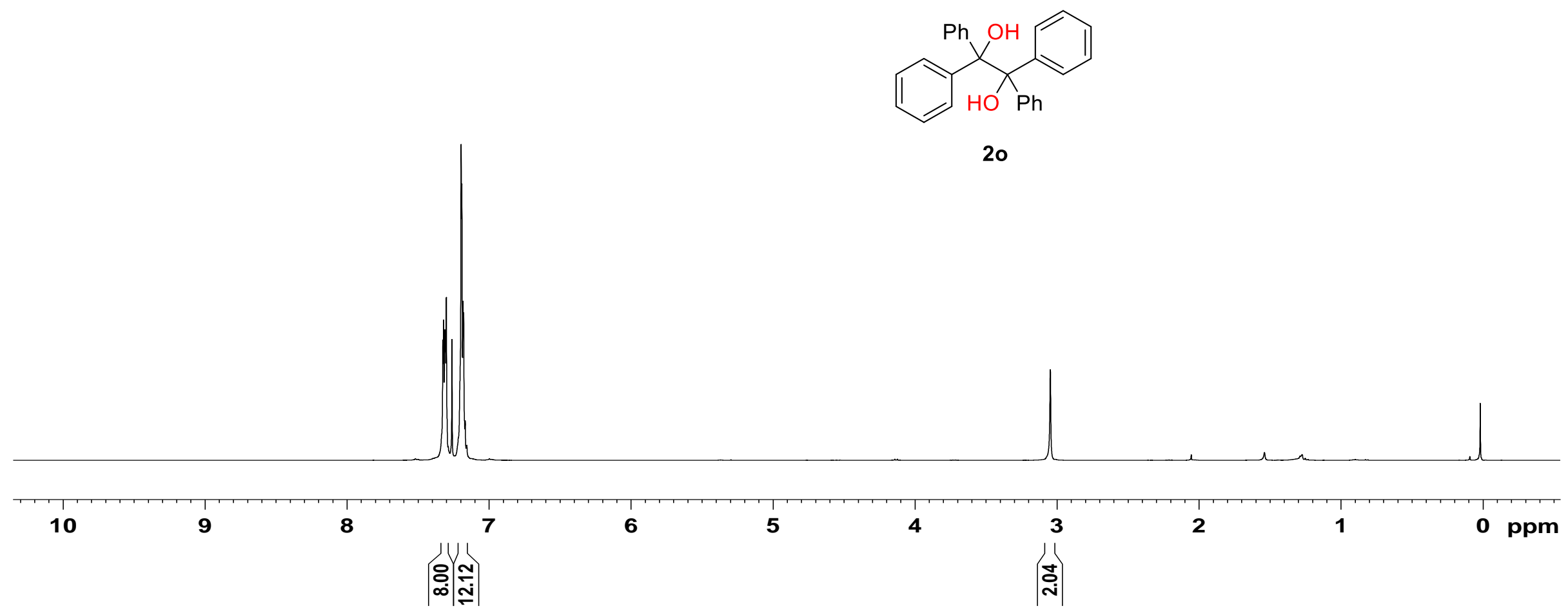

Figure S35. ${ }^{1} \mathrm{H}$ NMR spectra of $20\left(\mathrm{CDCl}_{3}, 400 \mathrm{M}\right)$. 


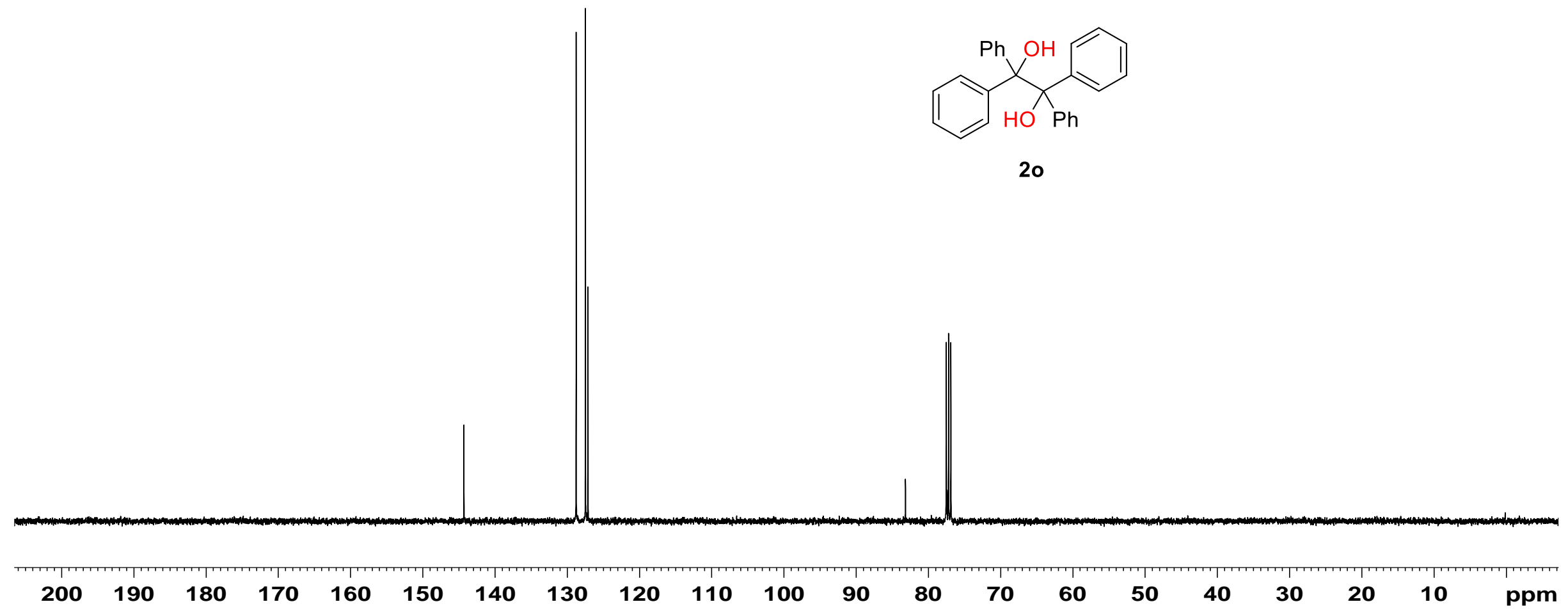

Figure S36. ${ }^{13} \mathrm{C}$ NMR spectra of $20\left(\mathrm{CDCl}_{3}, 100 \mathrm{M}\right)$. 

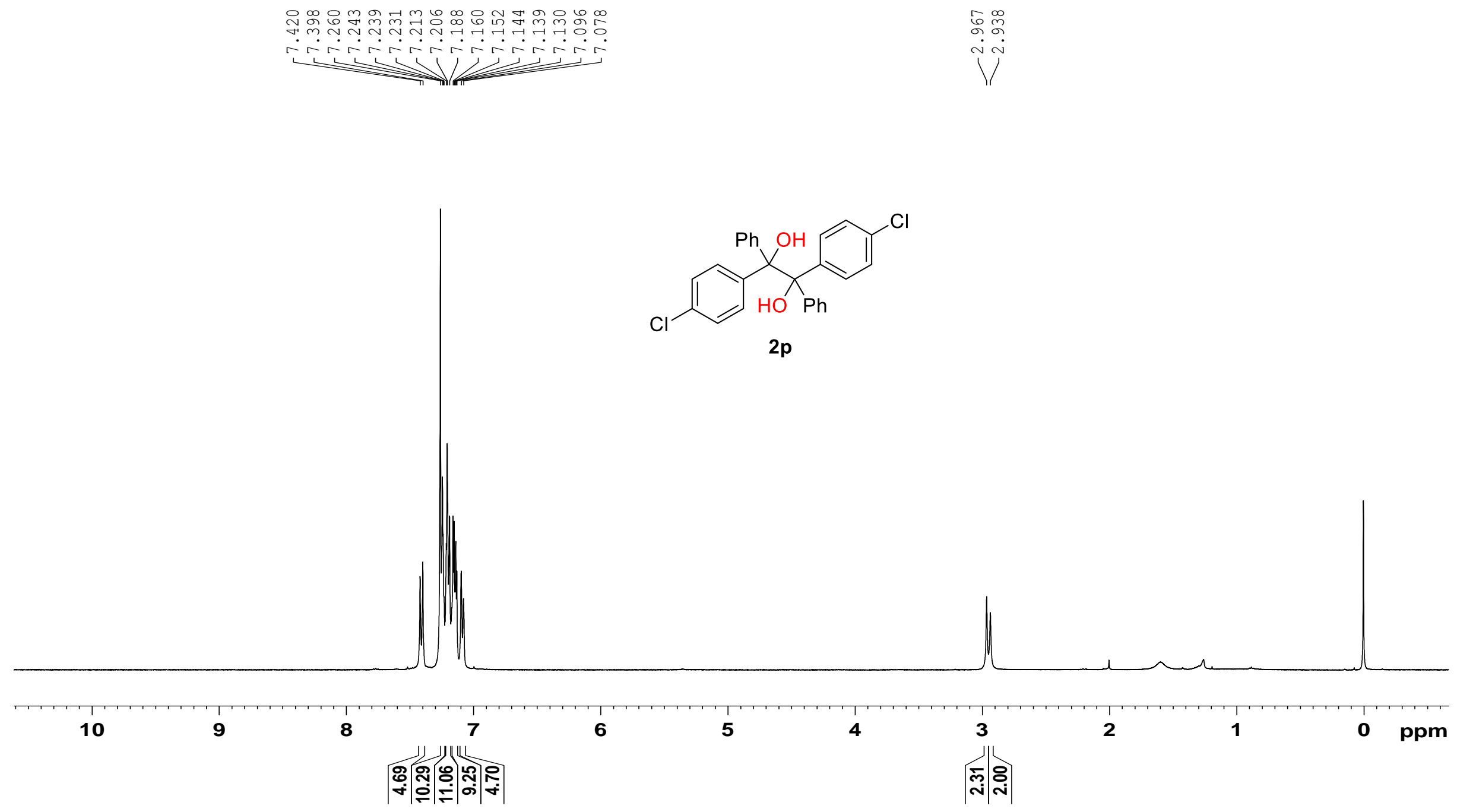

Figure S37. ${ }^{1} \mathrm{H}$ NMR spectra of $\mathbf{2 p}\left(\mathrm{CDCl}_{3}, 400 \mathrm{M}\right)$. 

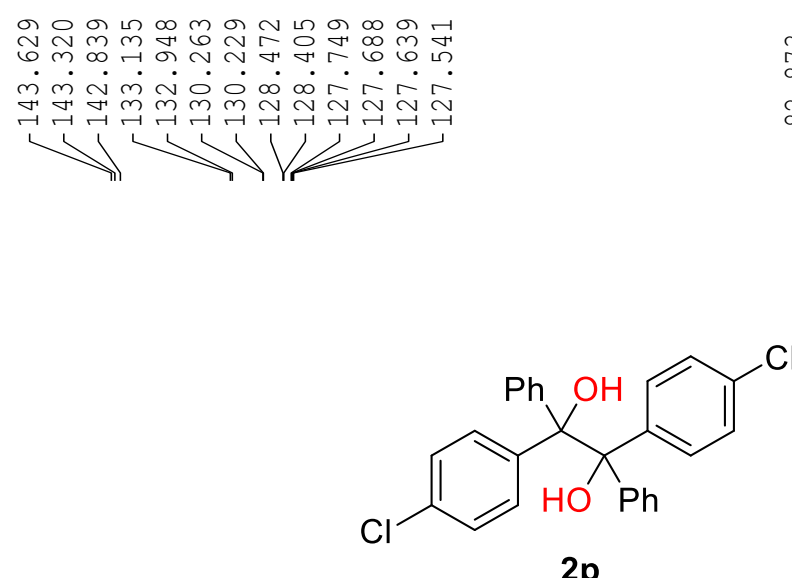

$2 p$

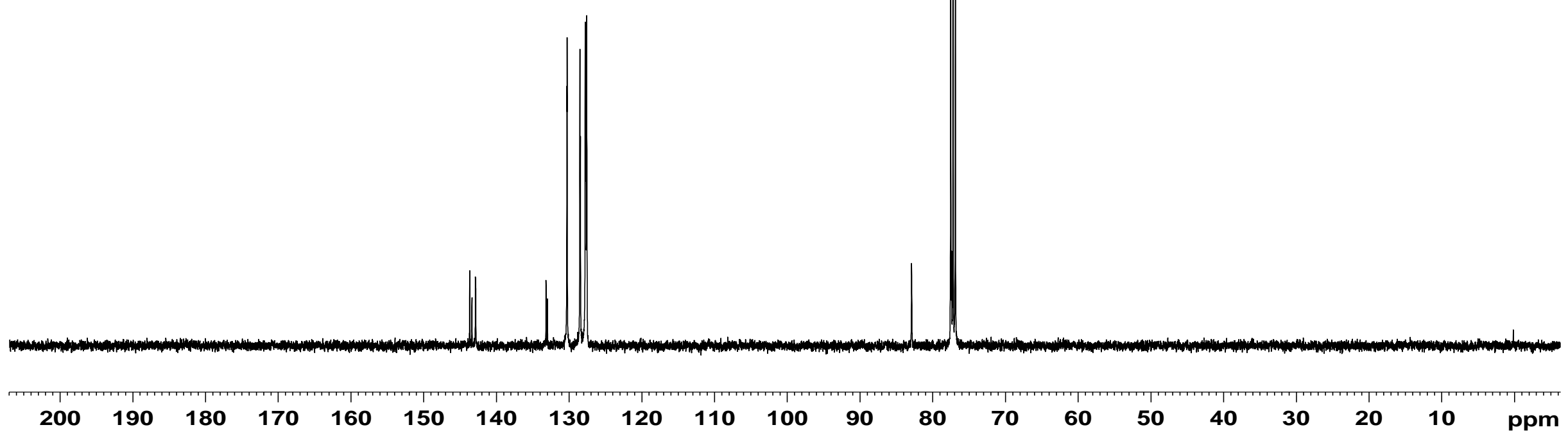

Figure S38. ${ }^{13} \mathrm{C}$ NMR spectra of $\mathbf{2} \mathbf{p}\left(\mathrm{CDCl}_{3}, 100 \mathrm{M}\right)$. 

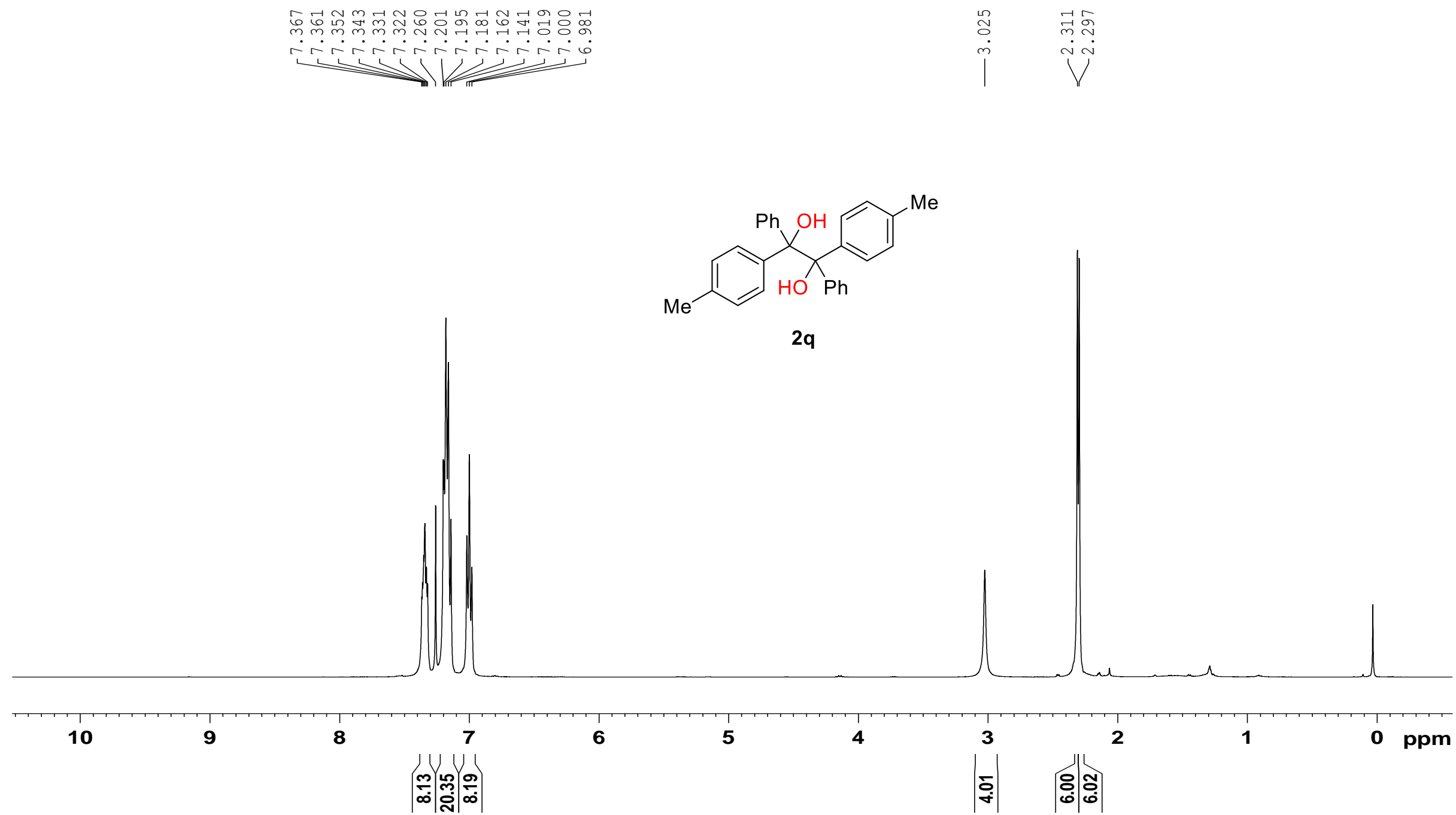

$2 q$
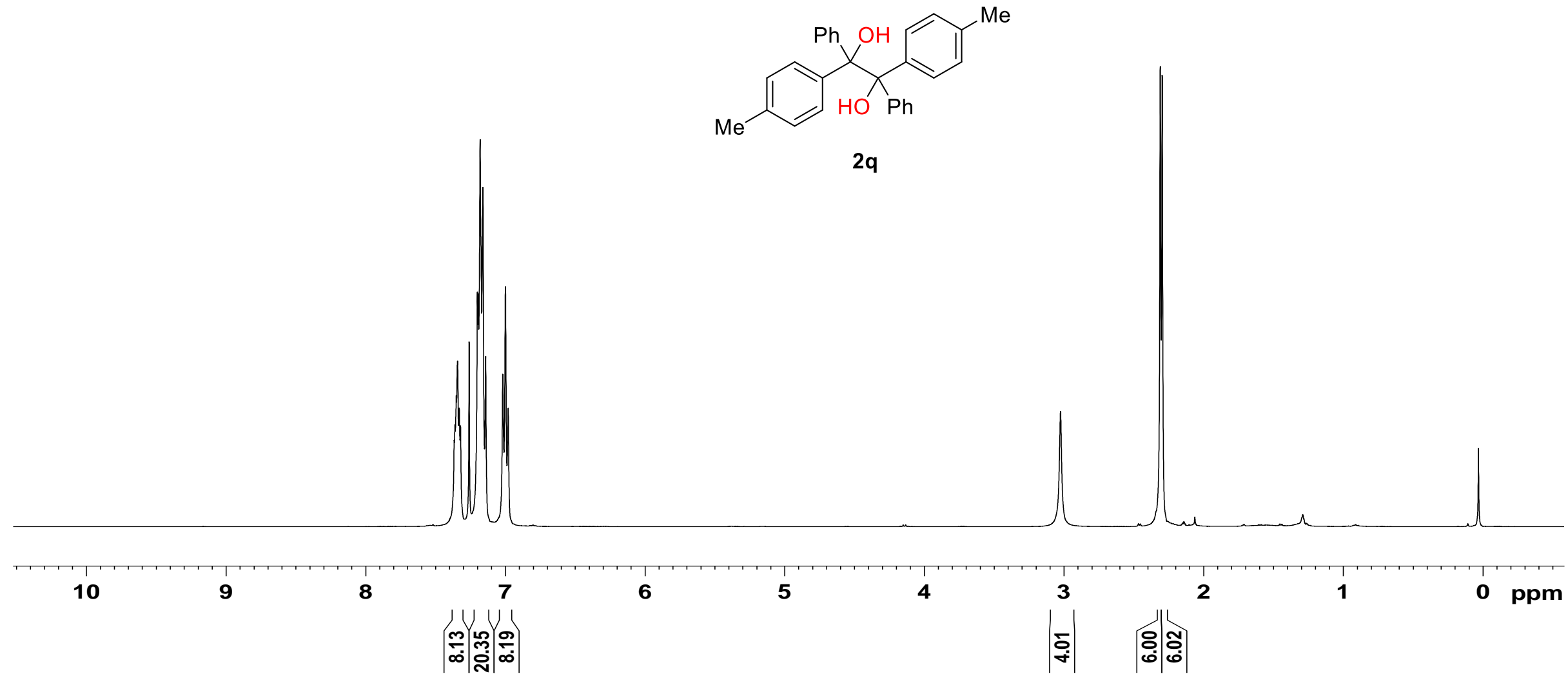

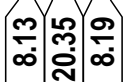

Figure S39. ${ }^{1} \mathrm{H}$ NMR spectra of $\mathbf{2 q}\left(\mathrm{CDCl}_{3}, 400 \mathrm{M}\right)$. 

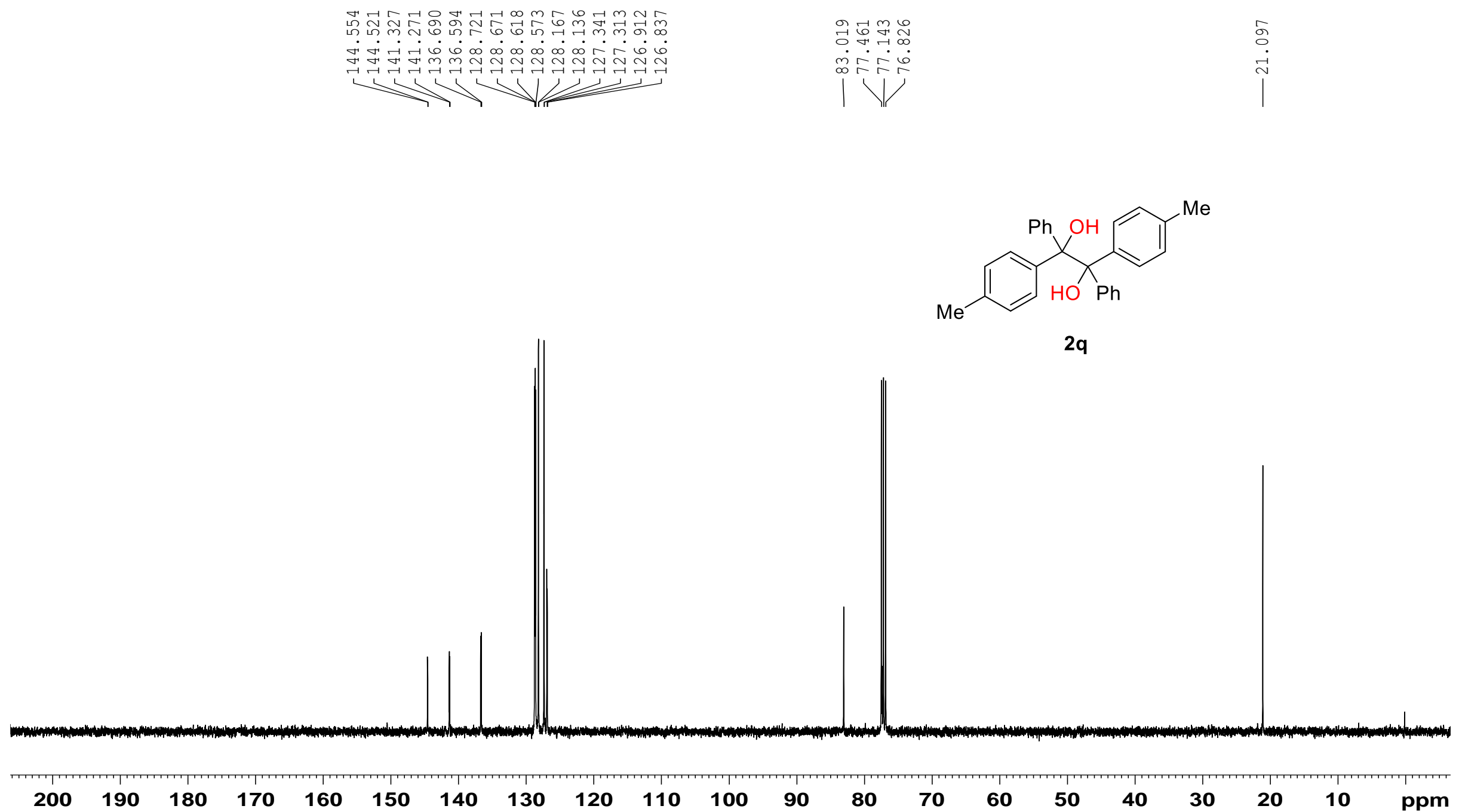

Figure S40. ${ }^{13} \mathrm{C}$ NMR spectra of $\mathbf{2 q}\left(\mathrm{CDCl}_{3}, 100 \mathrm{M}\right)$. 


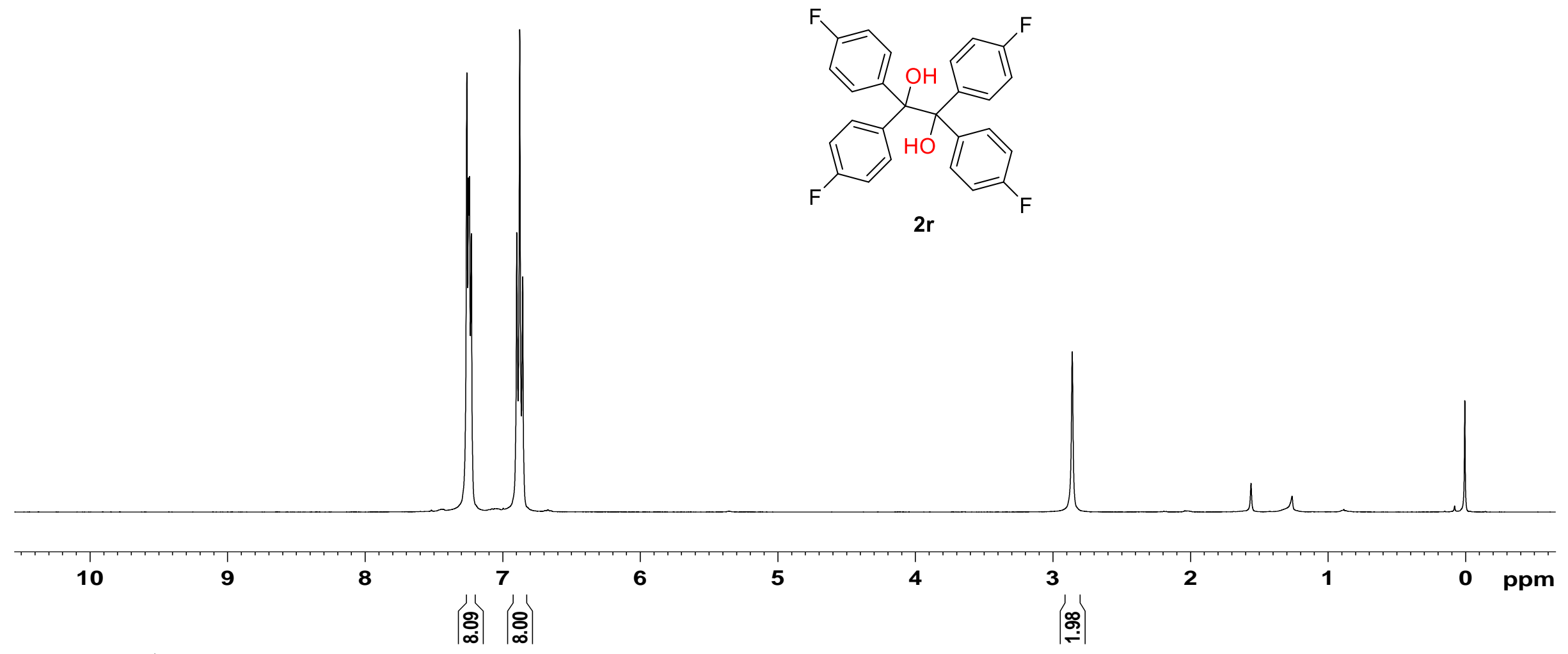

Figure S41. ${ }^{1} \mathrm{H}$ NMR spectra of $\mathbf{2 r}\left(\mathrm{CDCl}_{3}, 400 \mathrm{M}\right)$. 


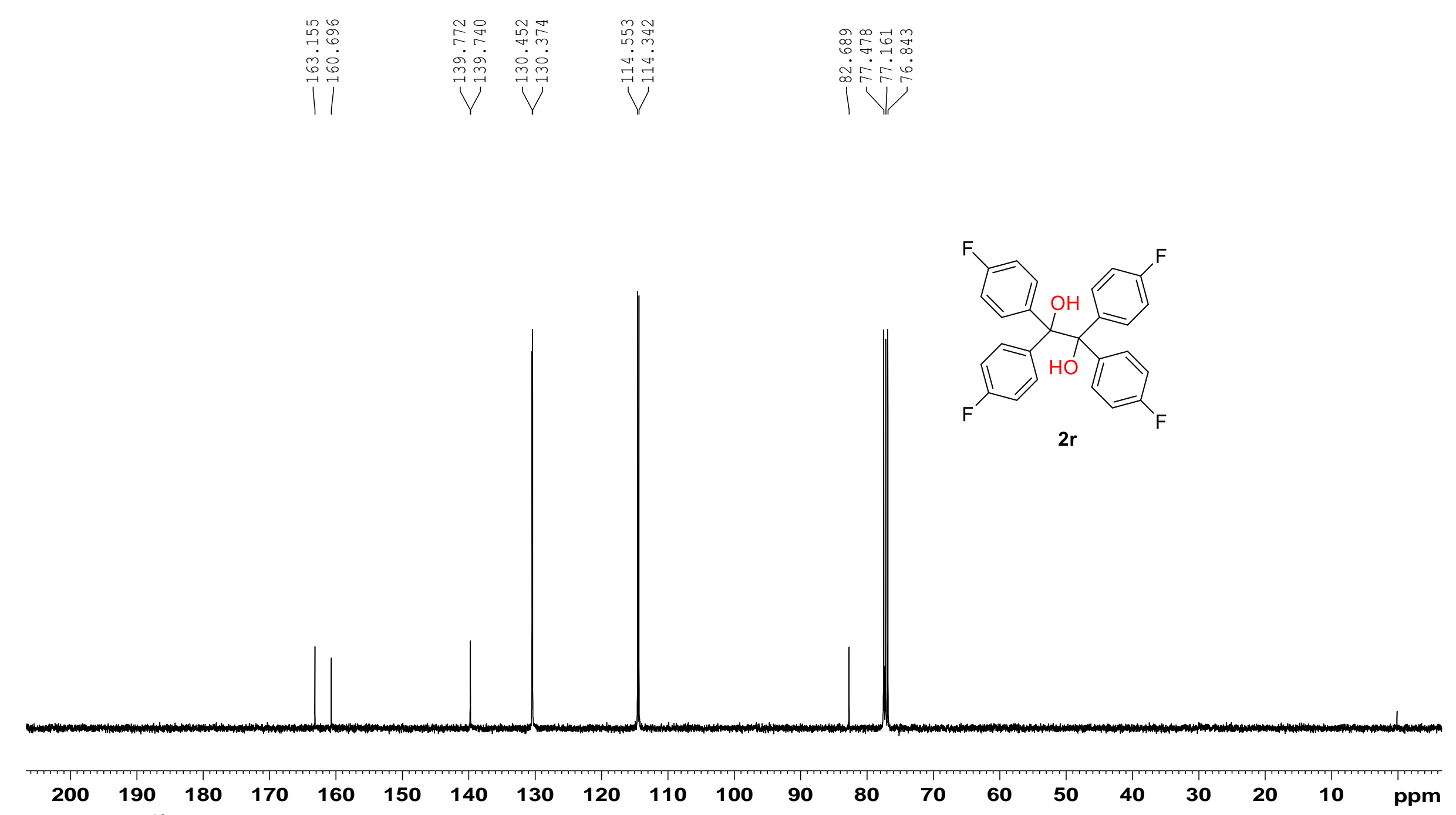

Figure $\mathbf{S 4 2} .{ }^{13} \mathrm{C}$ NMR spectra of $2 \mathbf{r}\left(\mathrm{CDCl}_{3}, 100 \mathrm{M}\right)$. 


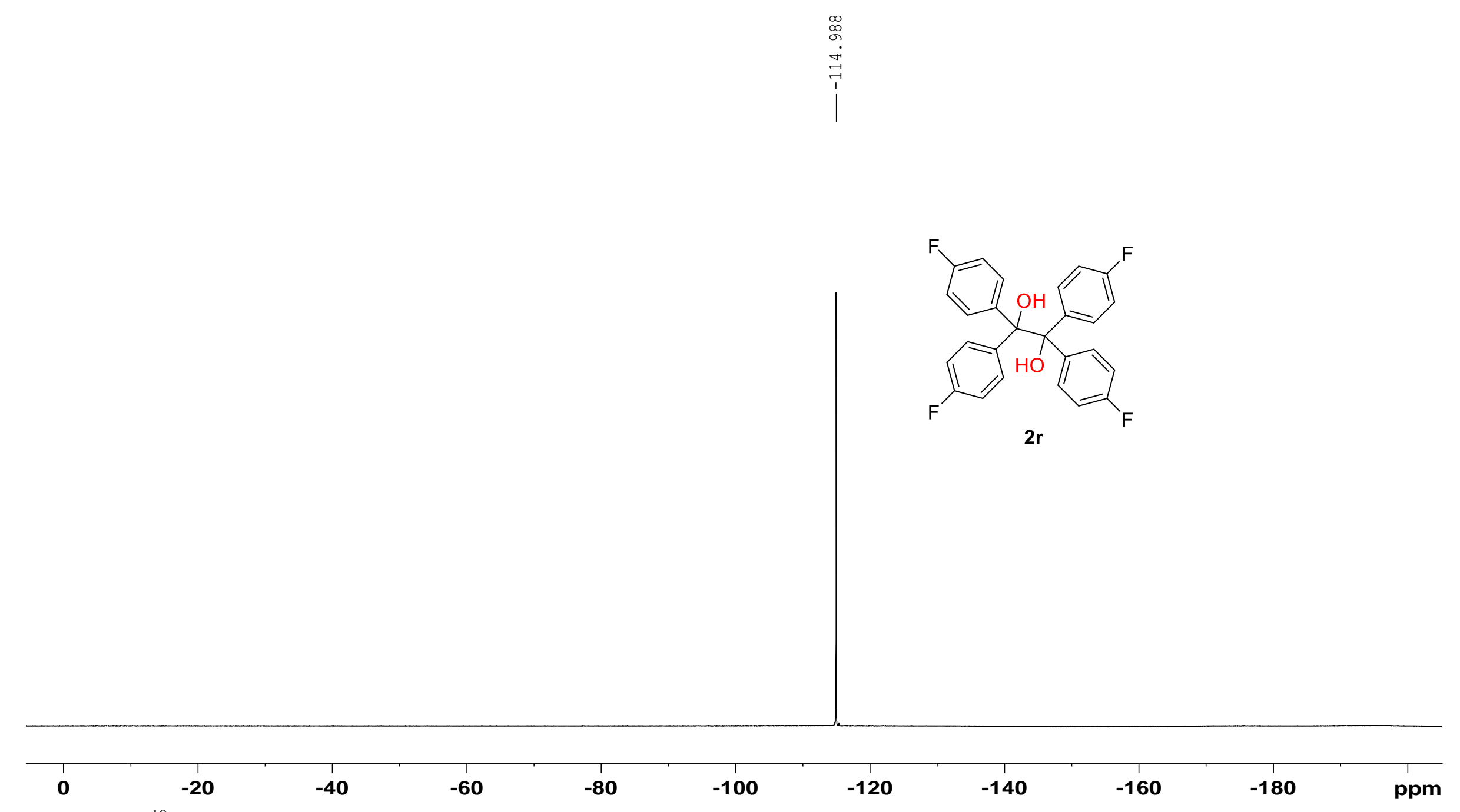

Figure S43. ${ }^{19} \mathrm{~F}$ NMR spectra of $\mathbf{2 r}\left(\mathrm{CDCl}_{3}, 376 \mathrm{M}\right)$. 


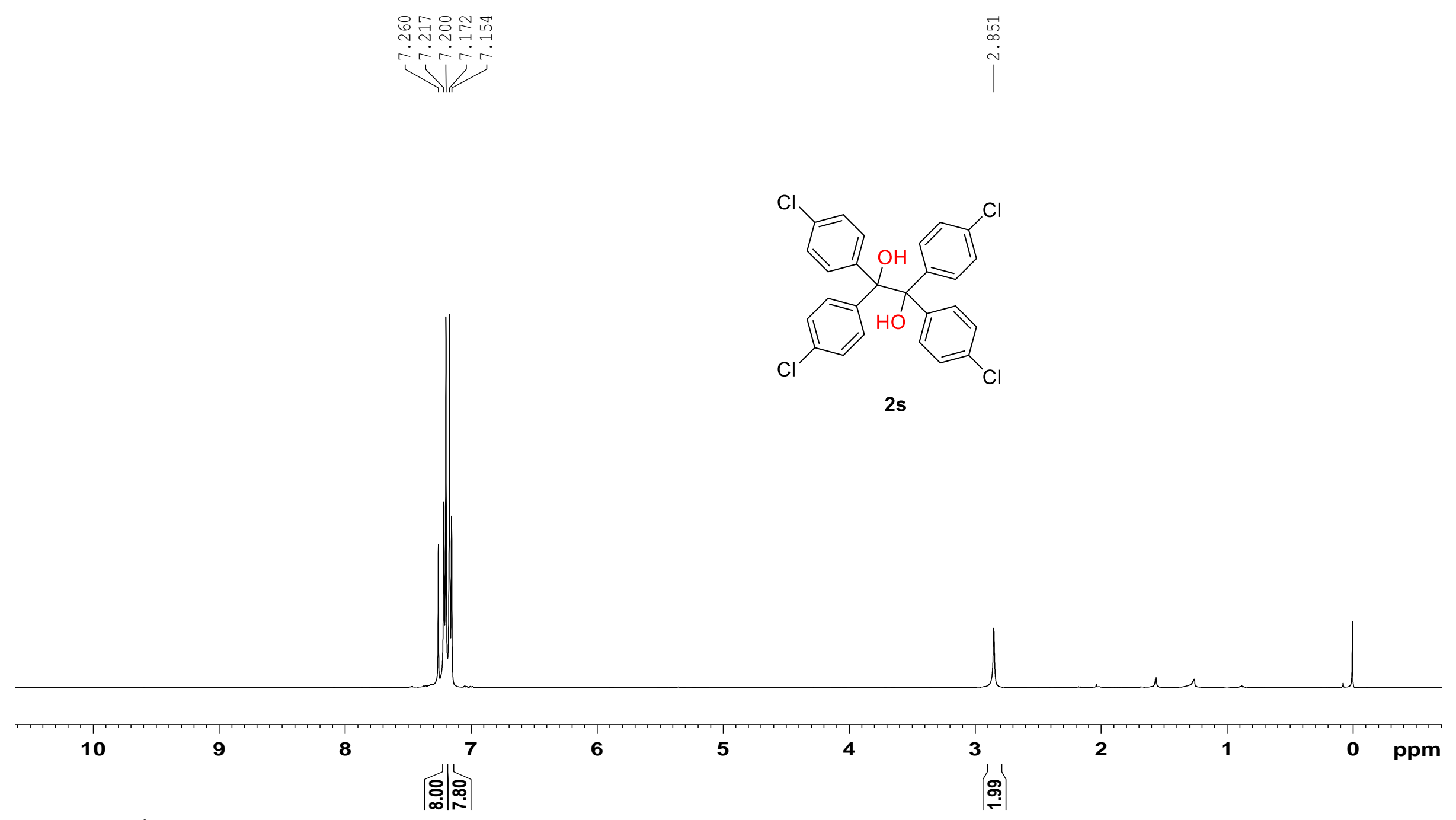

Figure S44. ${ }^{1} \mathrm{H}$ NMR spectra of $2 \mathrm{~s}\left(\mathrm{CDCl}_{3}, 400 \mathrm{M}\right)$. 

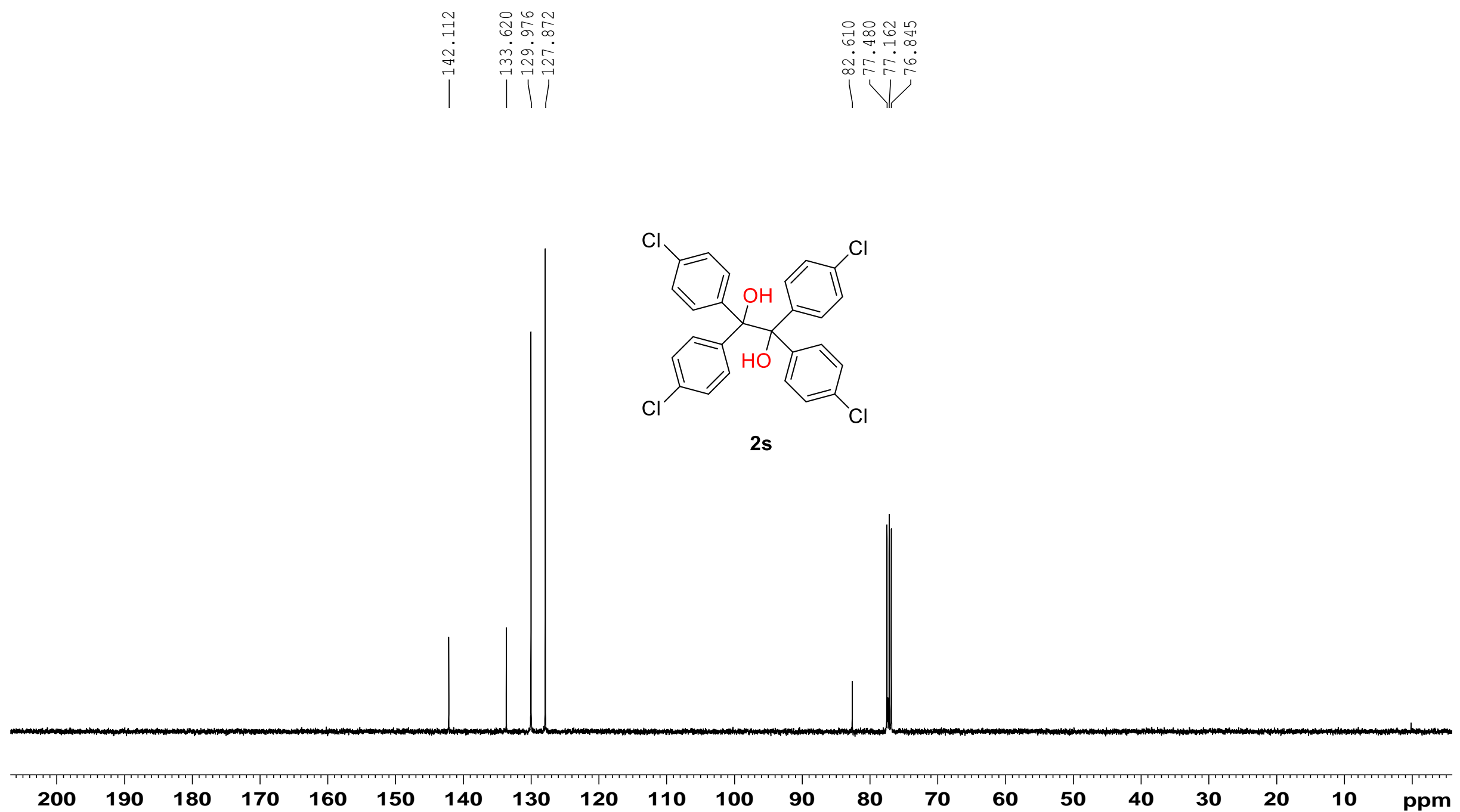

$2 s$

Figure S45. ${ }^{13} \mathrm{C}$ NMR spectra of $2 \mathrm{~s}\left(\mathrm{CDCl}_{3}, 100 \mathrm{M}\right)$. 


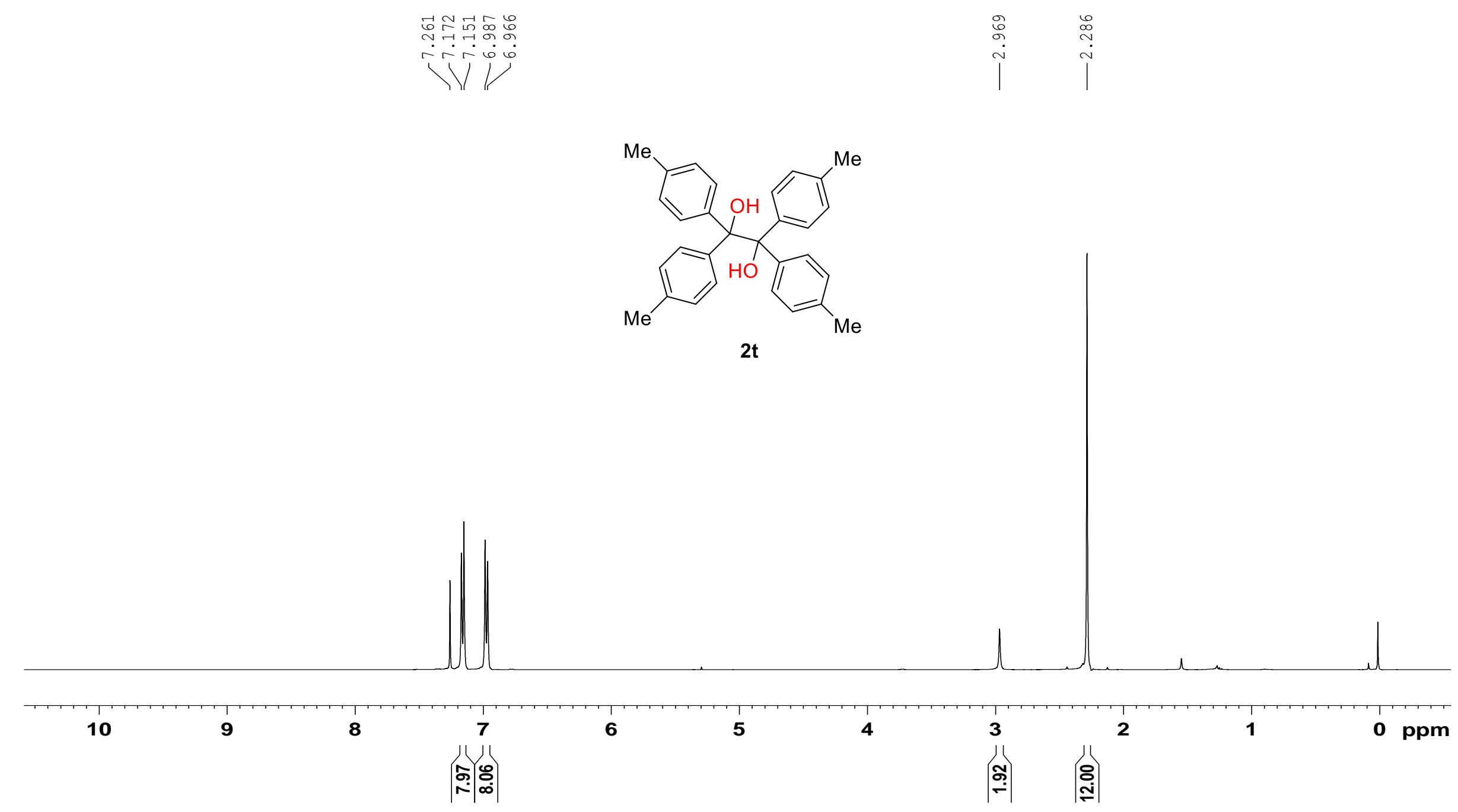

Figure S46. ${ }^{1} \mathrm{H}$ NMR spectra of $\mathbf{2 t}\left(\mathrm{CDCl}_{3}, 400 \mathrm{M}\right)$. 


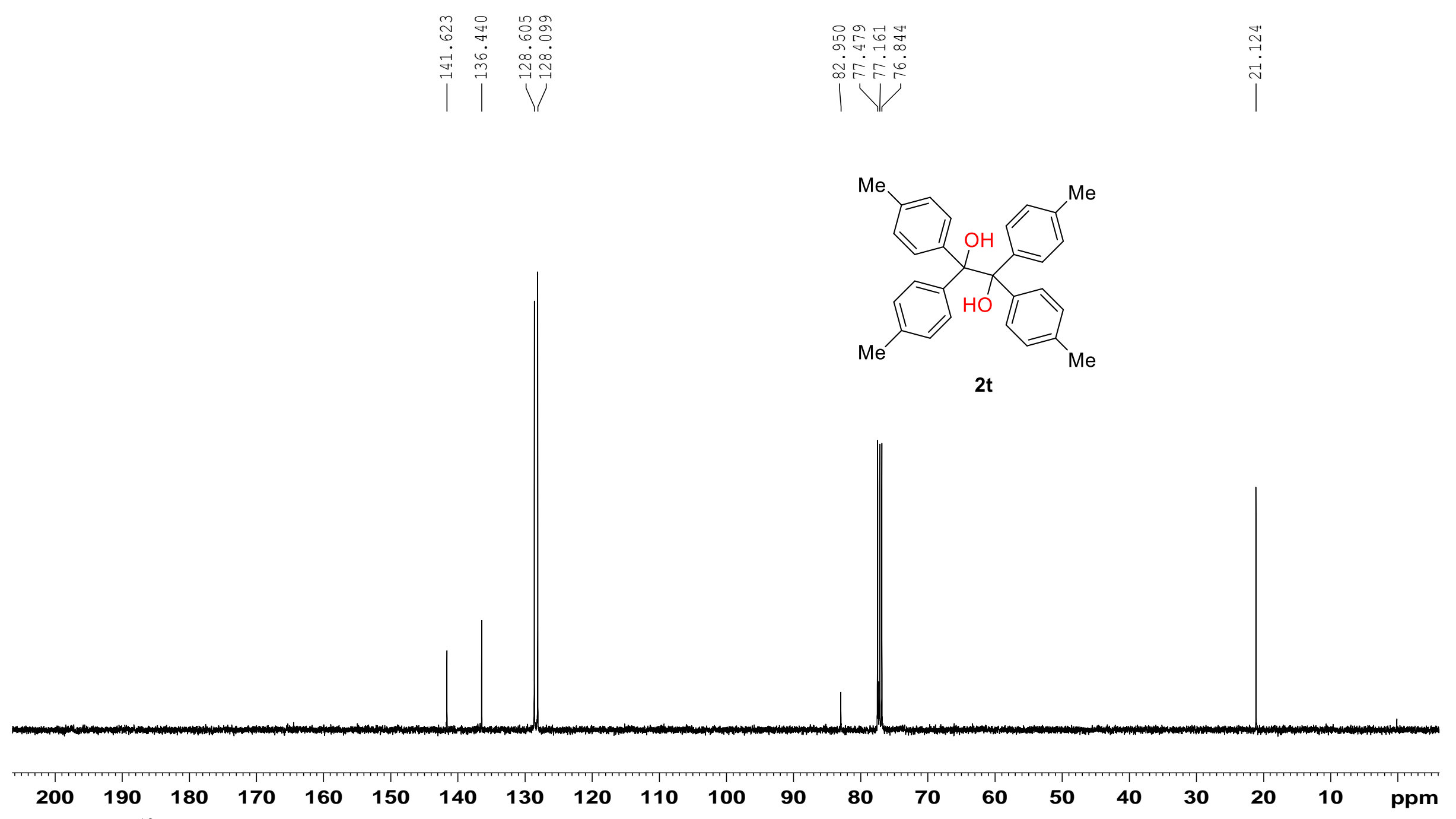

Figure S47. ${ }^{13} \mathrm{C}$ NMR spectra of $\mathbf{2 t}\left(\mathrm{CDCl}_{3}, 100 \mathrm{M}\right)$. 


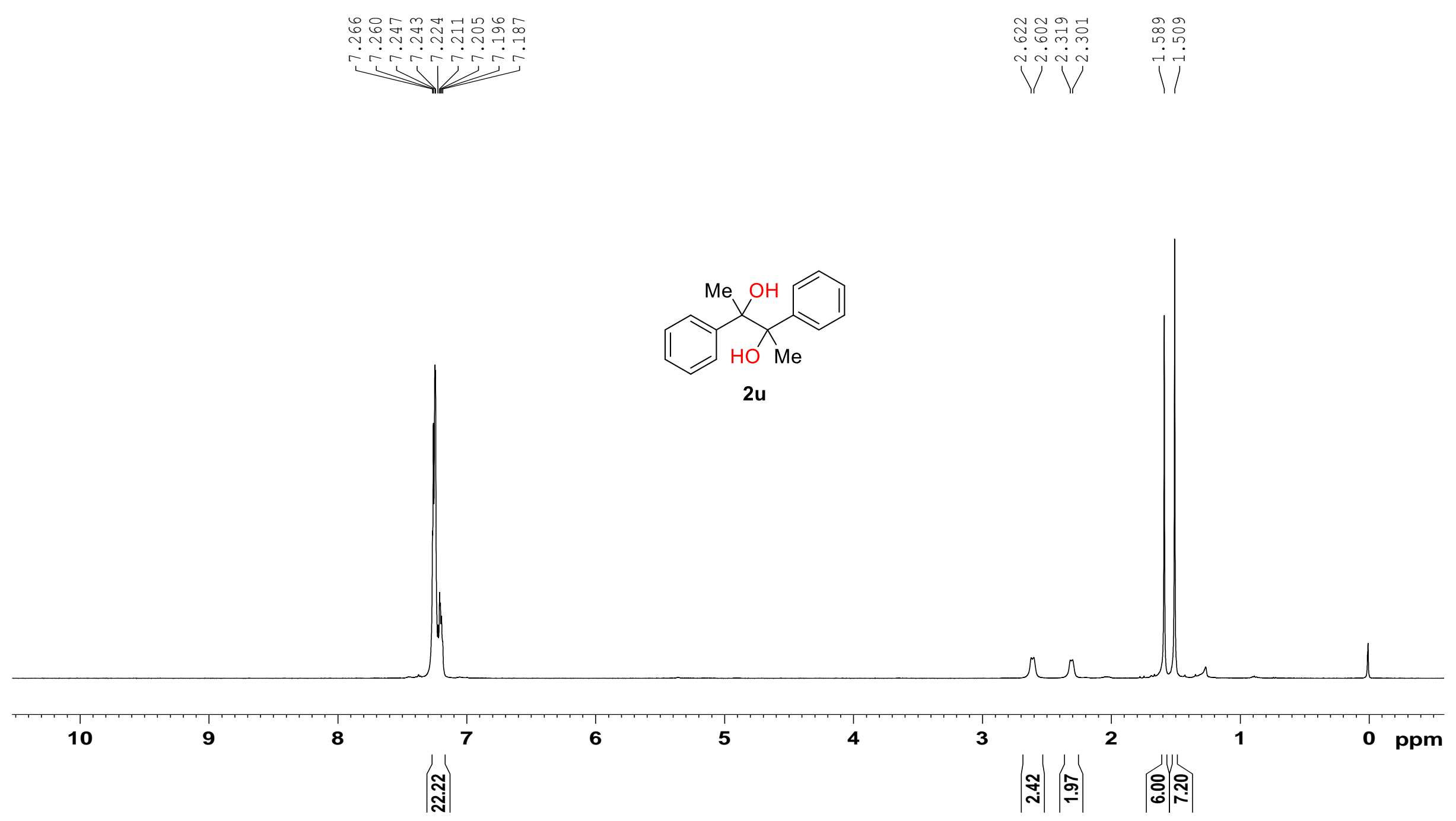

Figure S48. ${ }^{1} \mathrm{H}$ NMR spectra of $\mathbf{2 u}\left(\mathrm{CDCl}_{3}, 400 \mathrm{M}\right)$. 


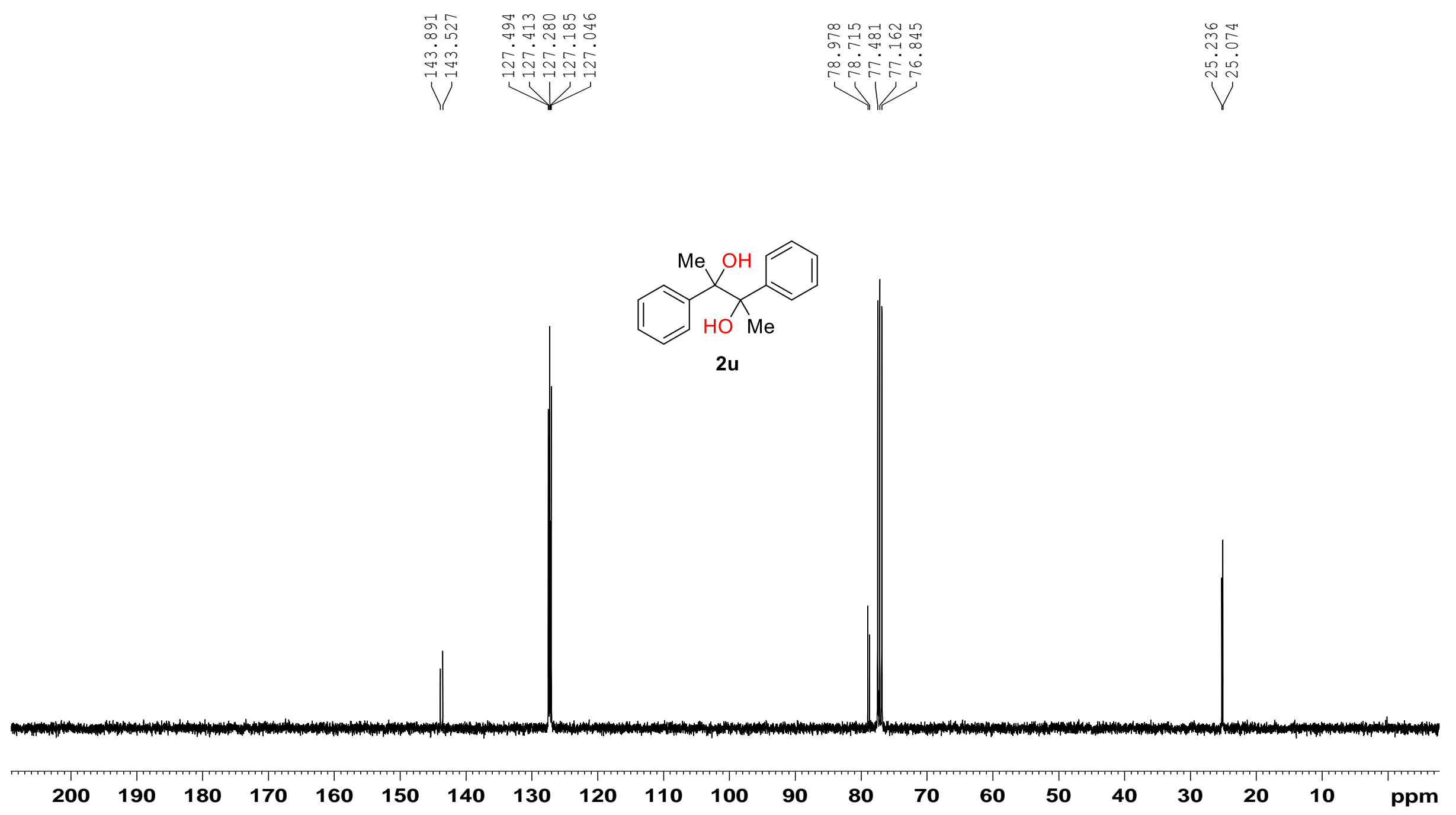

Figure S49. ${ }^{13} \mathrm{C}$ NMR spectra of $2 \mathbf{u}\left(\mathrm{CDCl}_{3}, 100 \mathrm{M}\right)$. 


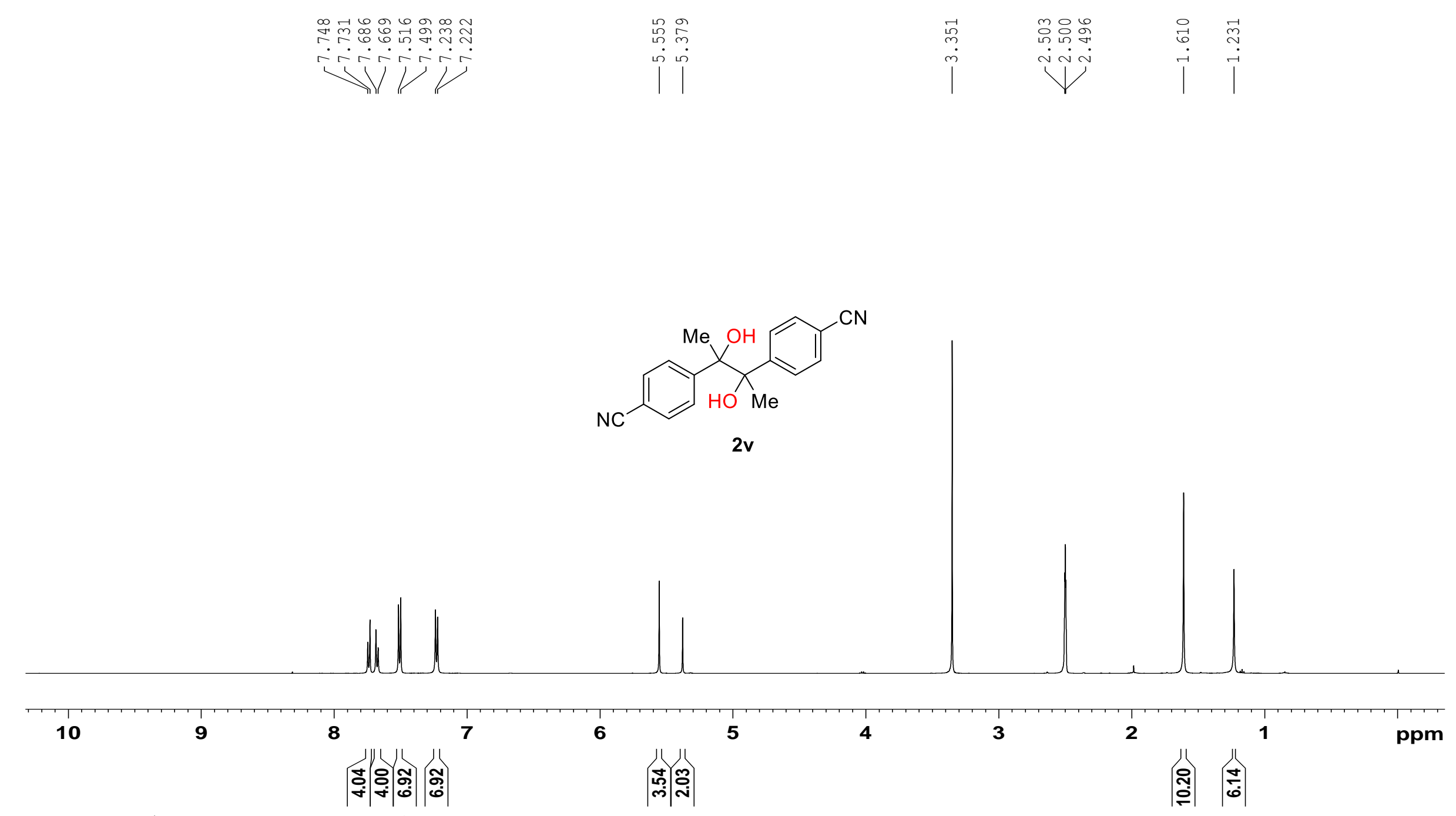

Figure S50. ${ }^{1} \mathrm{H}$ NMR spectra of $\mathbf{2 v}\left(d^{6}\right.$-DMSO, 500M). 

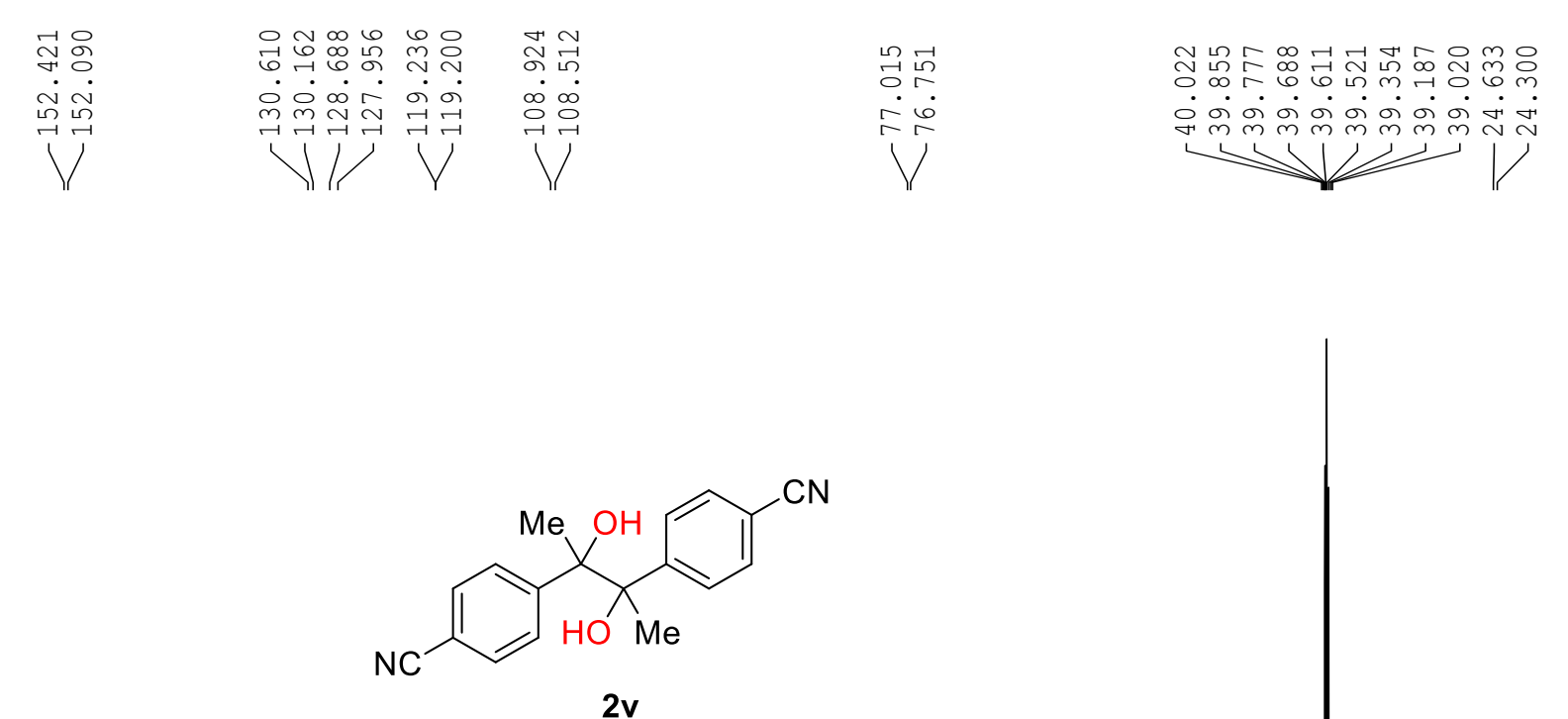

2v

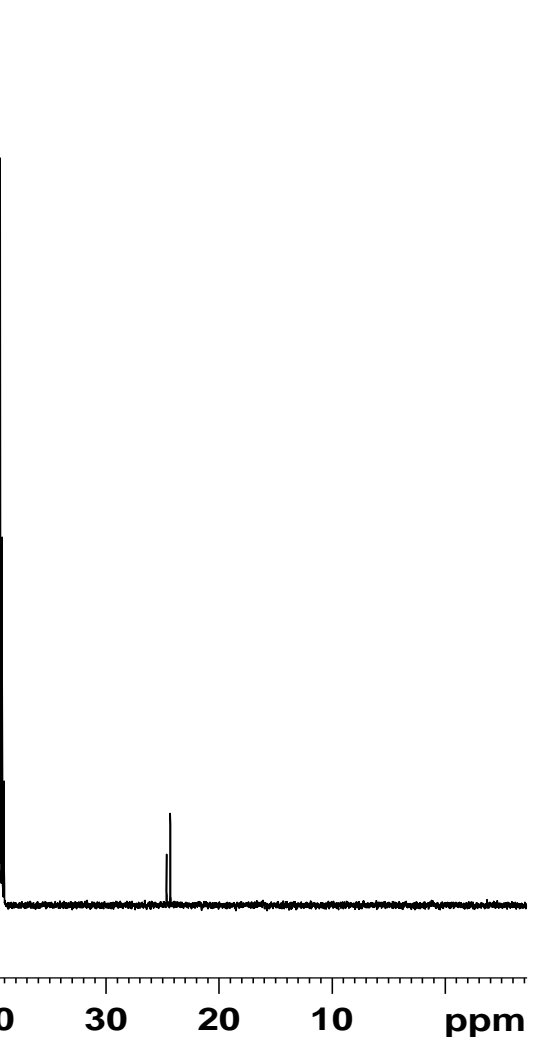

$\begin{array}{cccccccc}200 & 190 & 180 & 170 & 160 & 150 & 140 & 130 \\ \text { Figure S51. } & { }^{13} \mathrm{C} \text { NMR } & \text { spectra of } 2 \mathrm{v} & \left(d^{6} \text {-DMSO, }\right. & 125 \mathrm{M})\end{array}$ 


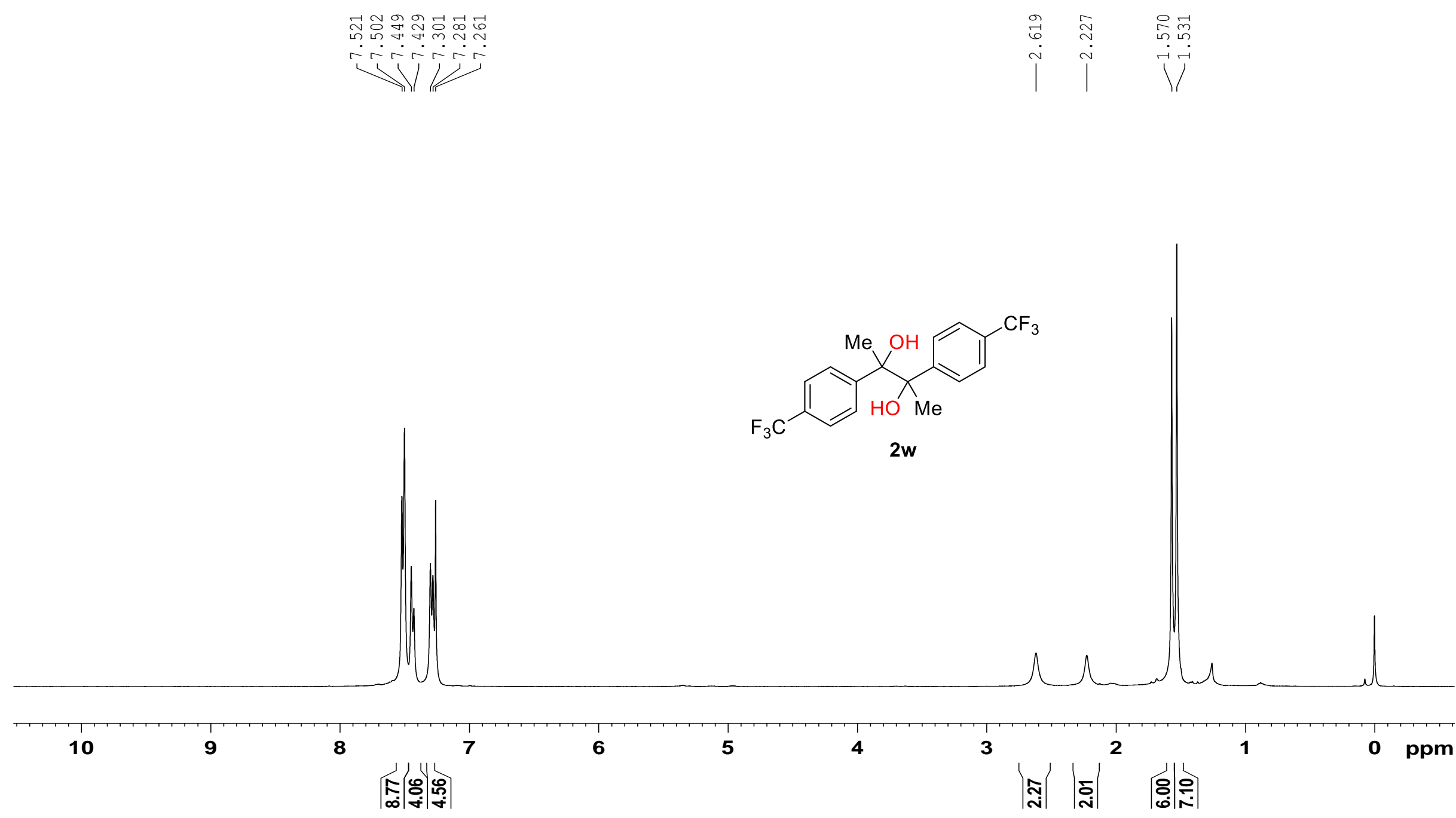

Figure S52. ${ }^{1} \mathrm{H}$ NMR spectra of $\mathbf{2 w}\left(\mathrm{CDCl}_{3}, 400 \mathrm{M}\right)$. 

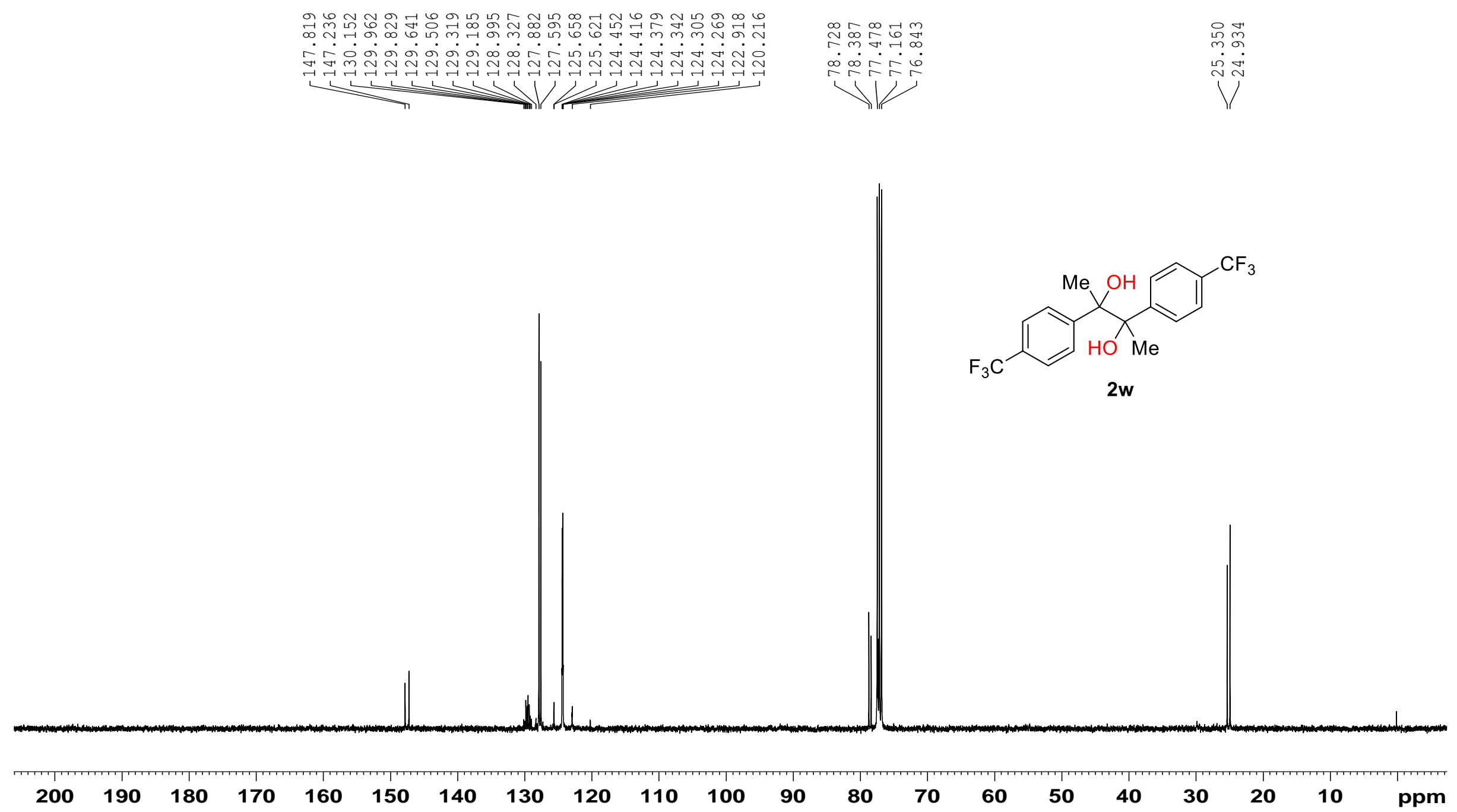

Figure S53. ${ }^{13} \mathrm{C}$ NMR spectra of $2 \mathbf{w}\left(\mathrm{CDCl}_{3}, 100 \mathrm{M}\right)$. 


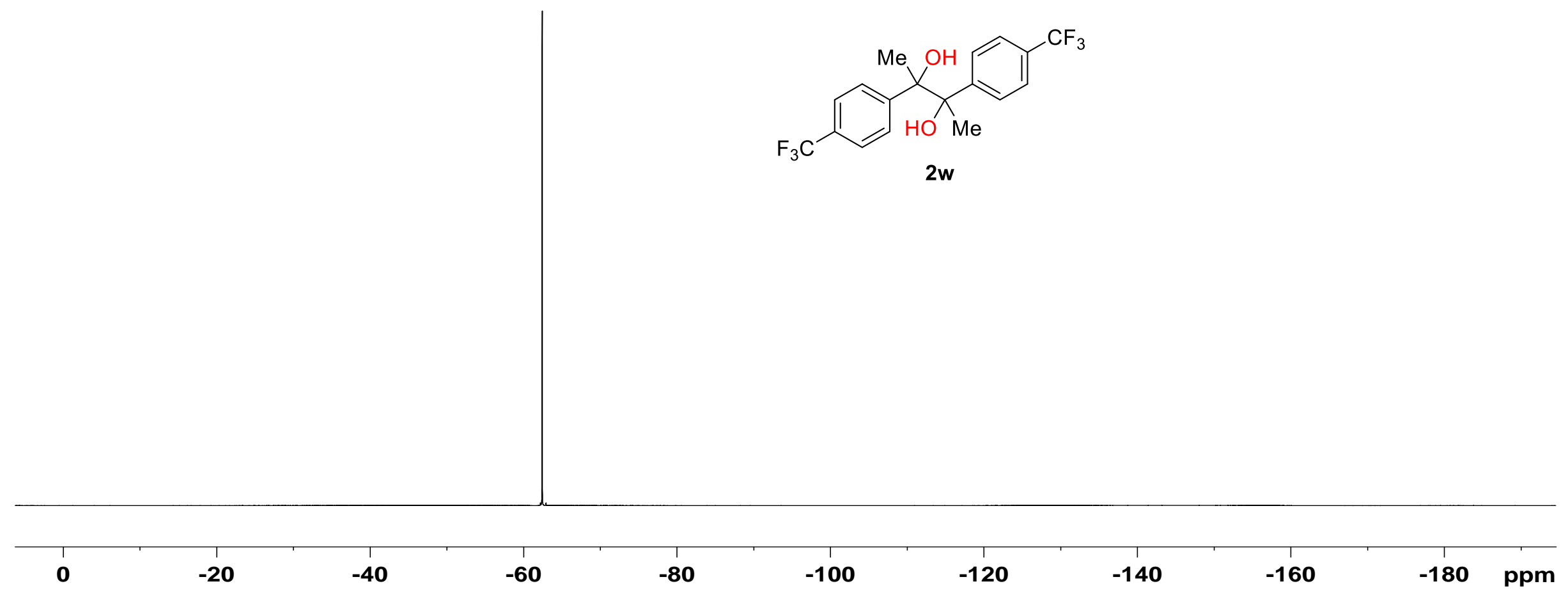

Figure S54. ${ }^{19} \mathrm{~F}$ NMR spectra of $\mathbf{2 w}\left(\mathrm{CDCl}_{3}, 376 \mathrm{M}\right)$. 


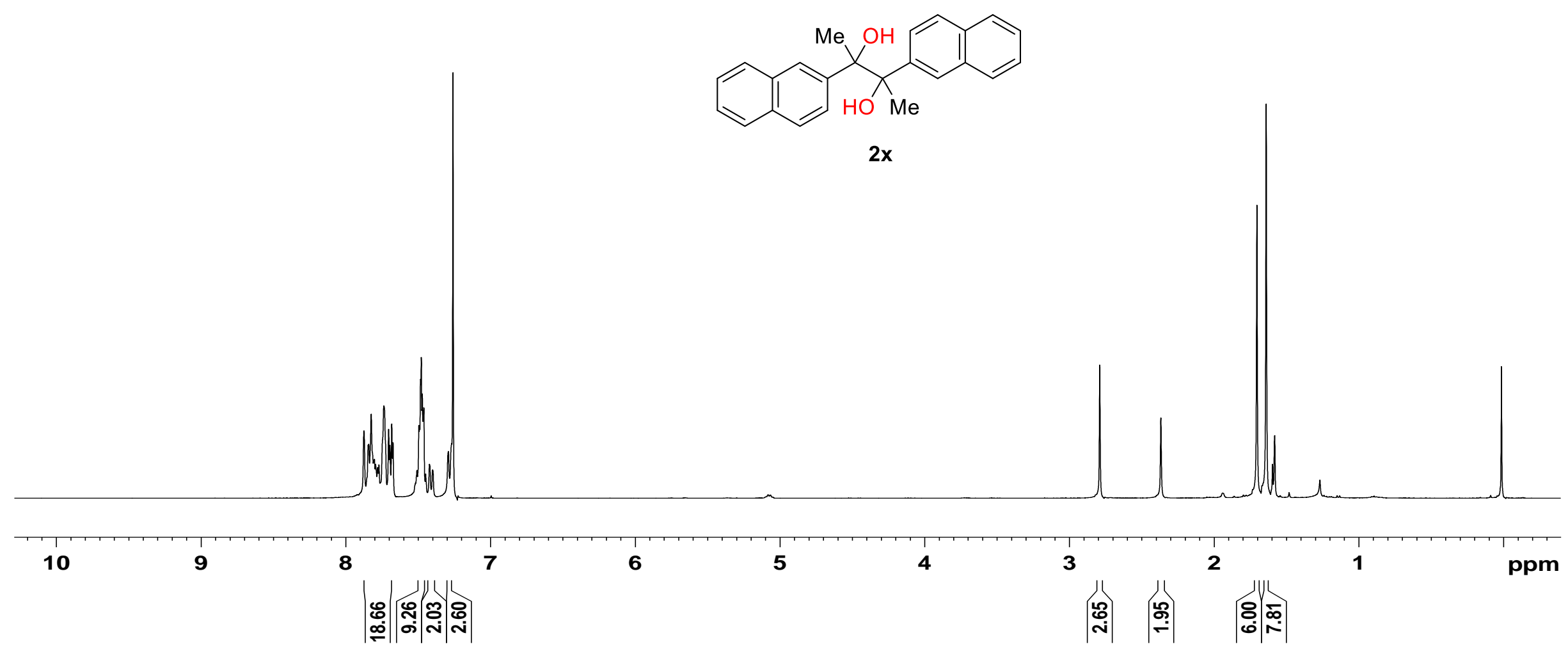

Figure S55. ${ }^{1} \mathrm{H}$ NMR spectra of $\mathbf{2 x}\left(\mathrm{CDCl}_{3}, 400 \mathrm{M}\right)$. 

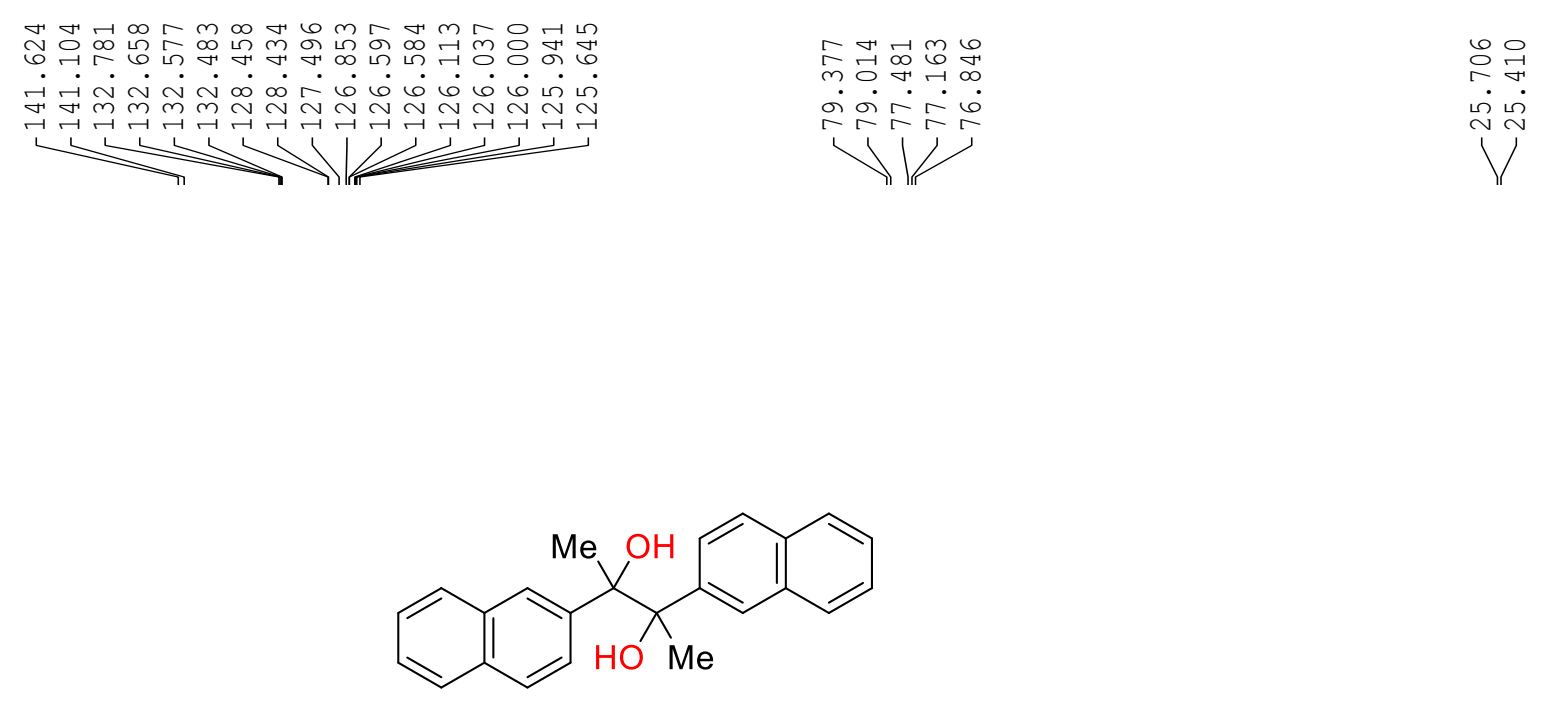

$2 x$

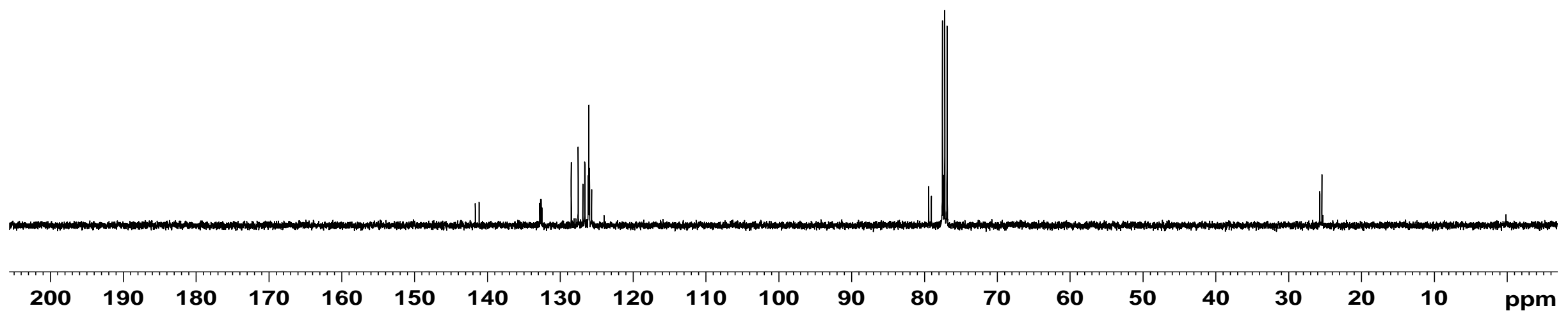

Figure S56. ${ }^{13} \mathrm{C}$ NMR spectra of $\mathbf{2 x}\left(\mathrm{CDCl}_{3}, 100 \mathrm{M}\right)$. 


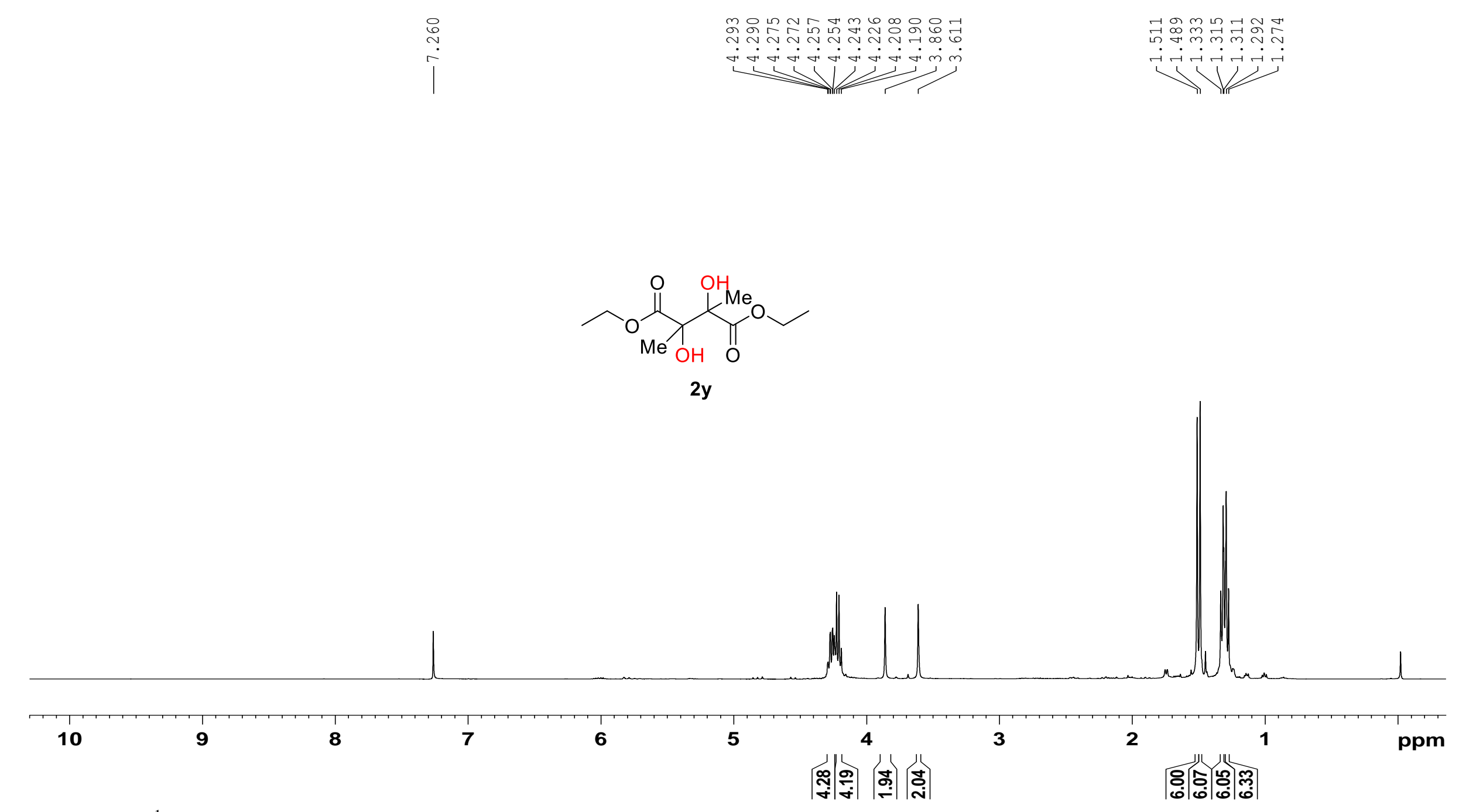

Figure S57. ${ }^{1} \mathrm{H}$ NMR spectra of $\mathbf{2 y}\left(\mathrm{CDCl}_{3}, 400 \mathrm{M}\right)$. 

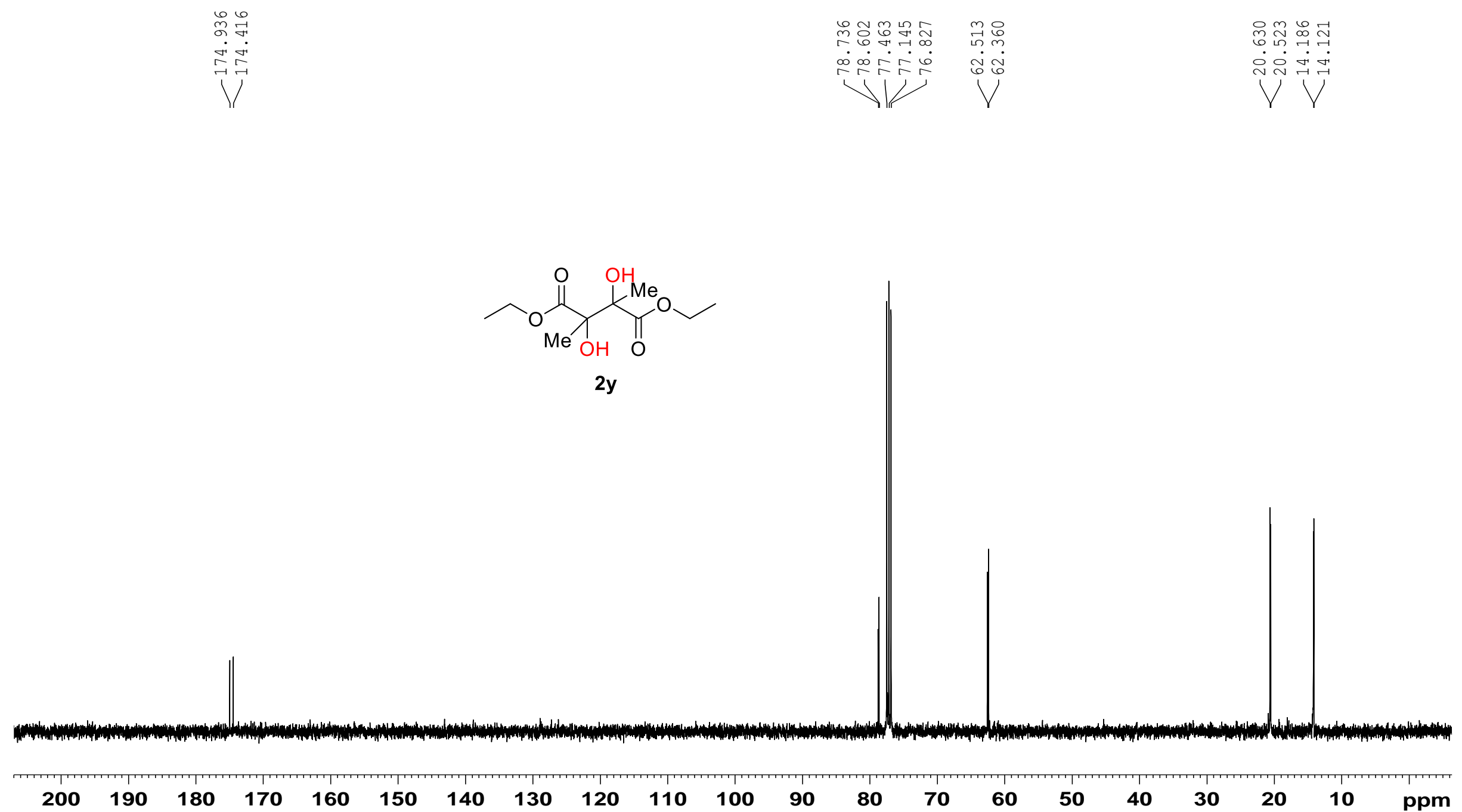

Figure S58. ${ }^{13} \mathrm{C}$ NMR spectra of $\mathbf{2 y}\left(\mathrm{CDCl}_{3}, 100 \mathrm{M}\right)$. 


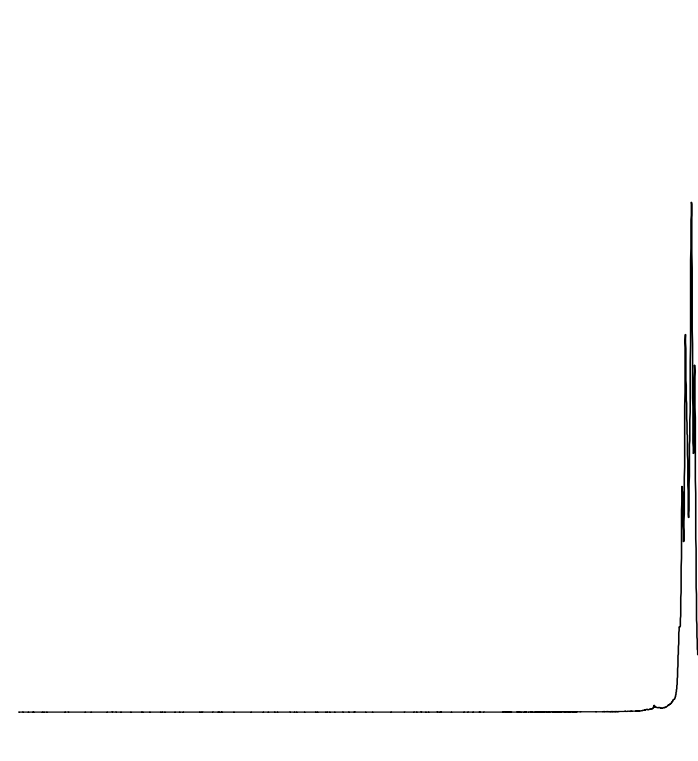

8

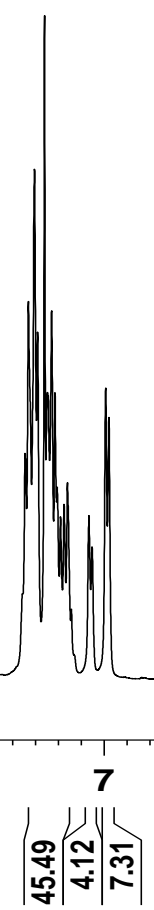

Figure S59. ${ }^{1} \mathrm{H}$ NMR spectra of $\mathbf{4 a}\left(\mathrm{CDCl}_{3}, 500 \mathrm{M}\right)$.

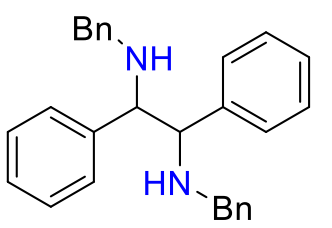

$4 a$

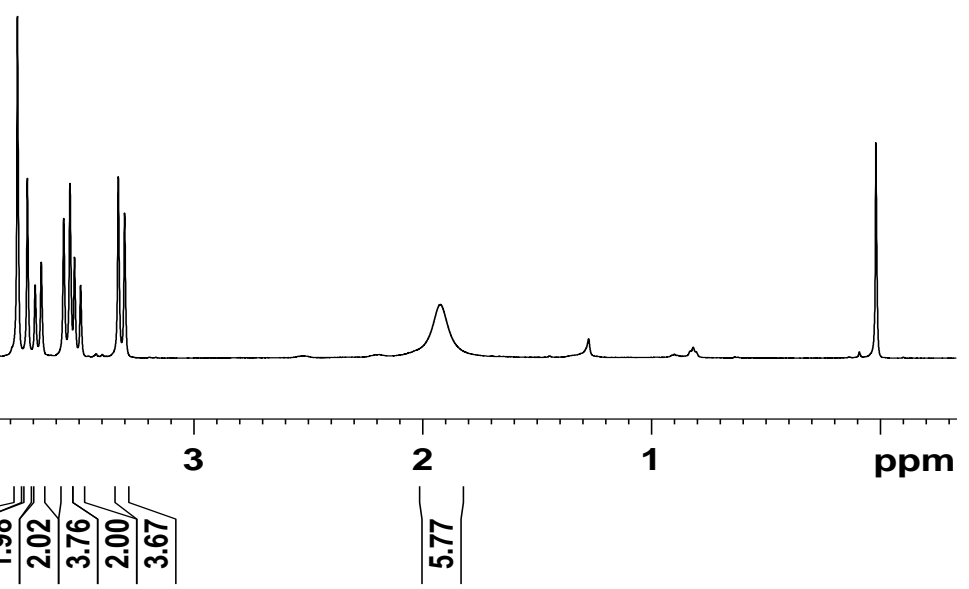



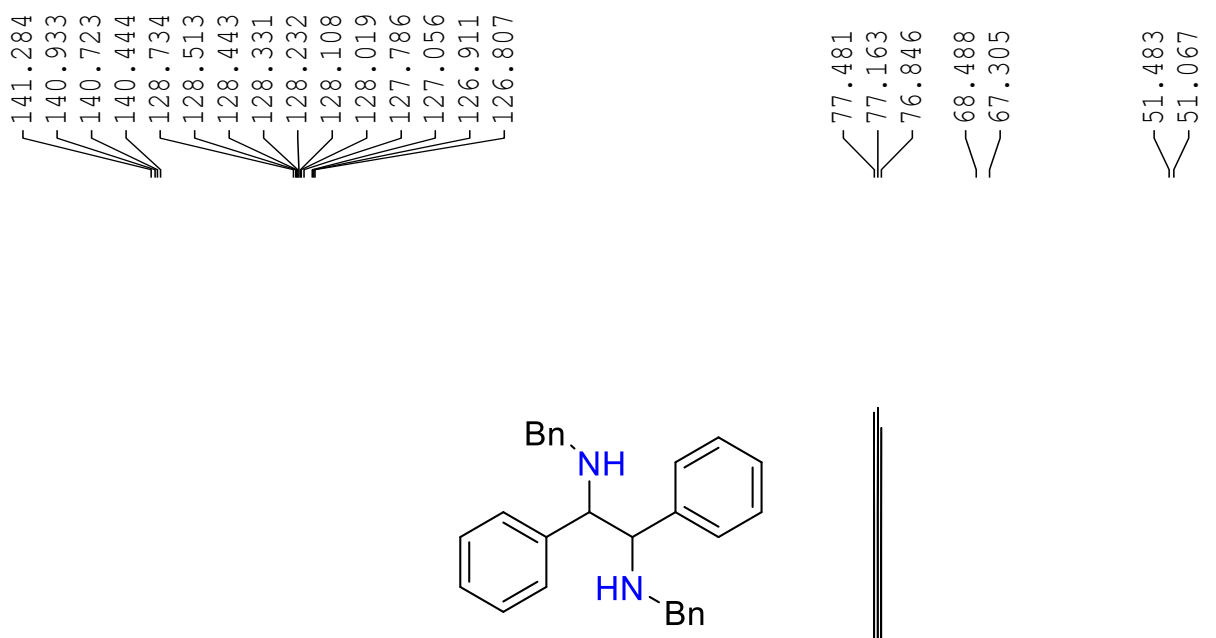

$4 a$

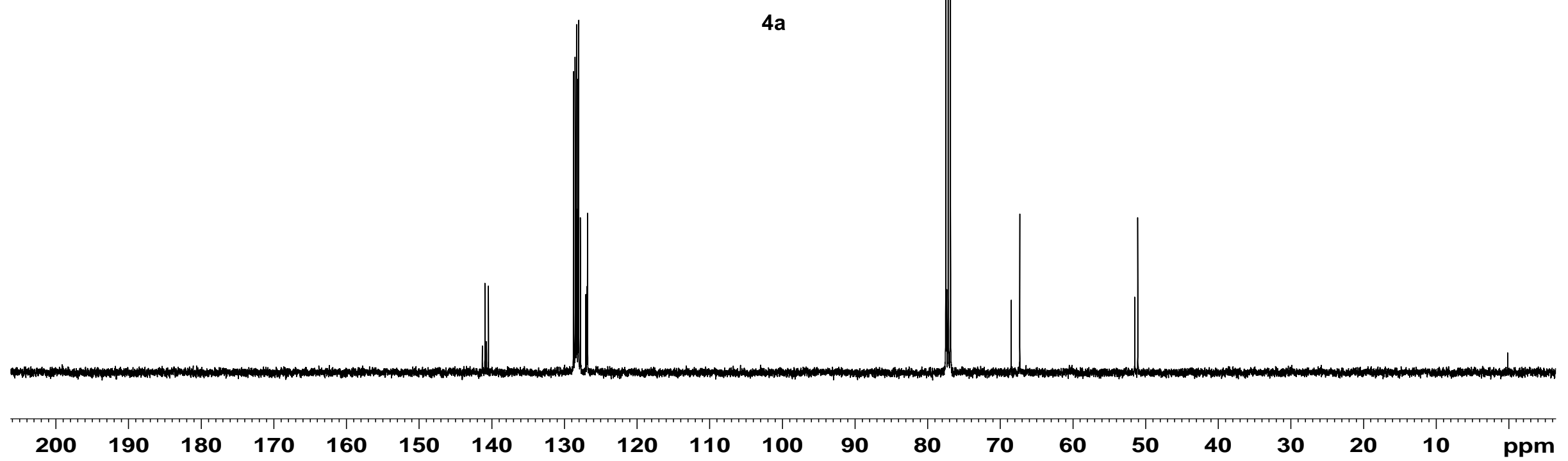

Figure S60. ${ }^{13} \mathrm{C}$ NMR spectra of $\mathbf{4 a}\left(\mathrm{CDCl}_{3}, 125 \mathrm{M}\right)$. 

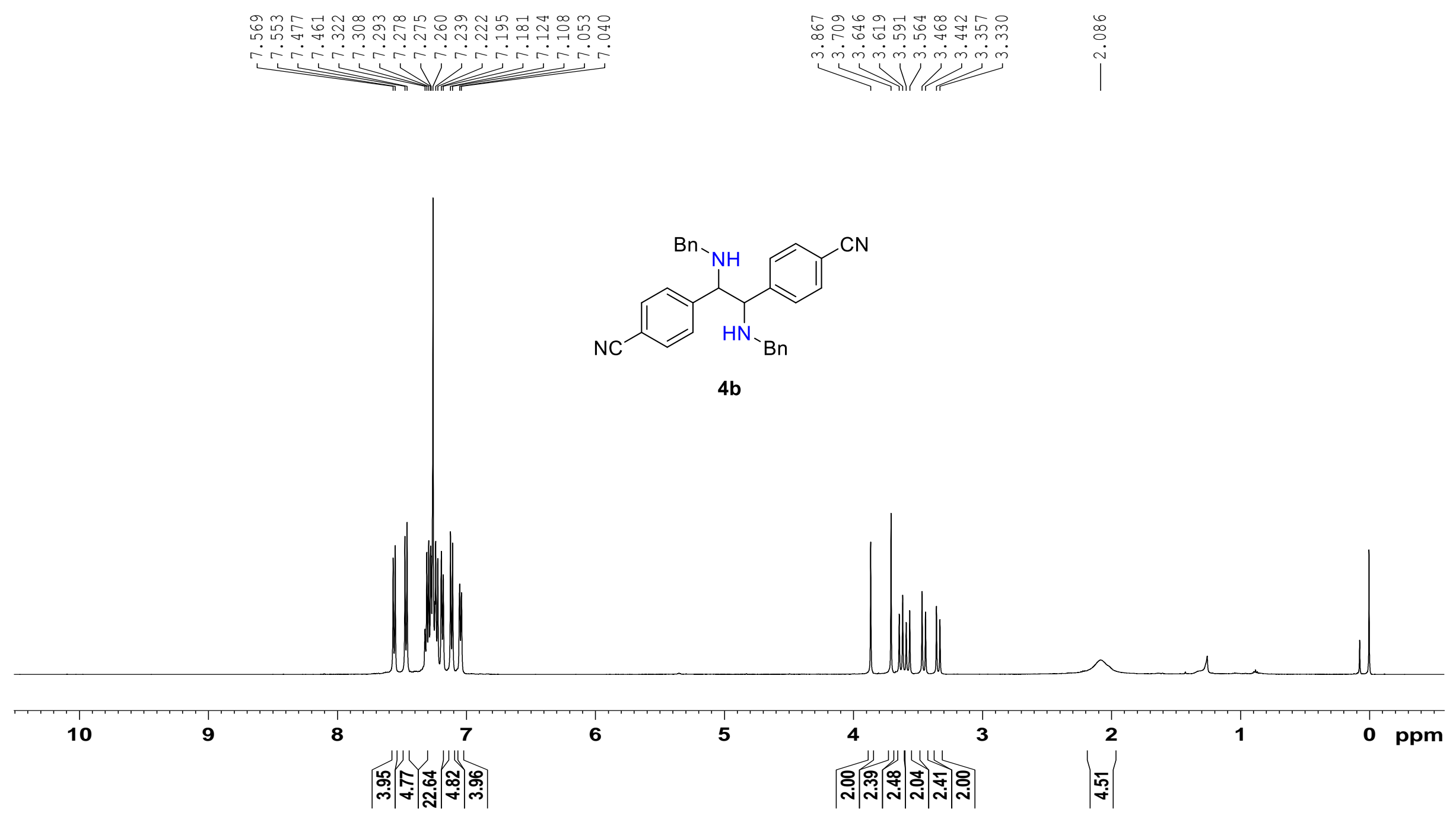

Figure S61. ${ }^{1} \mathrm{H}$ NMR spectra of $\mathbf{4 b}\left(\mathrm{CDCl}_{3}, 500 \mathrm{M}\right)$. 

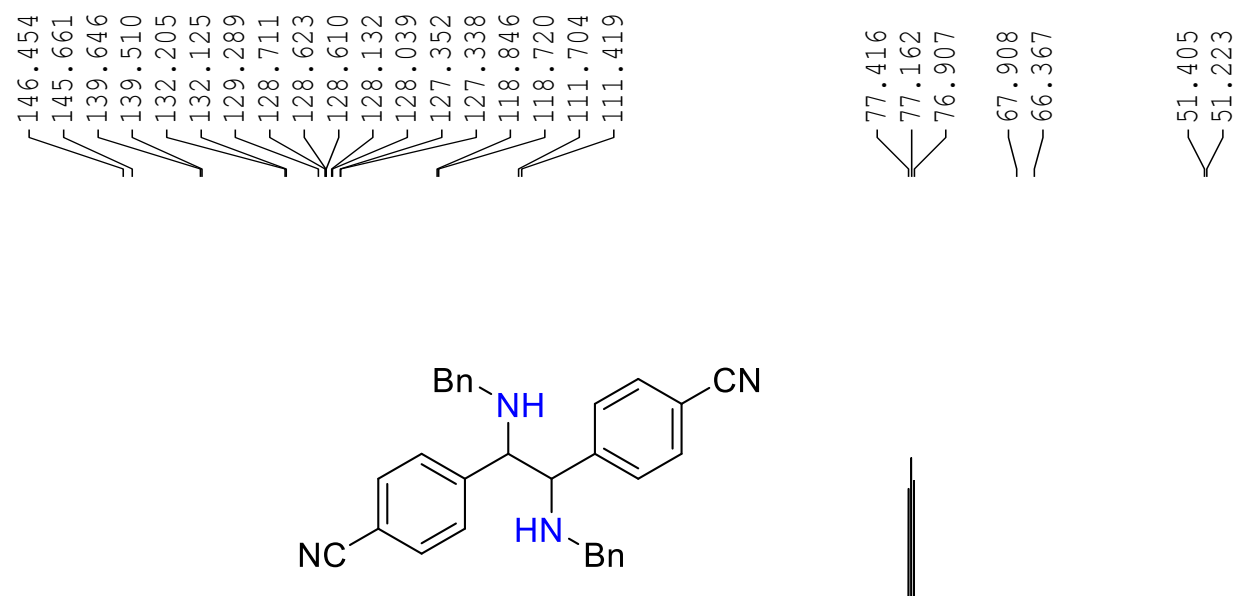

4b

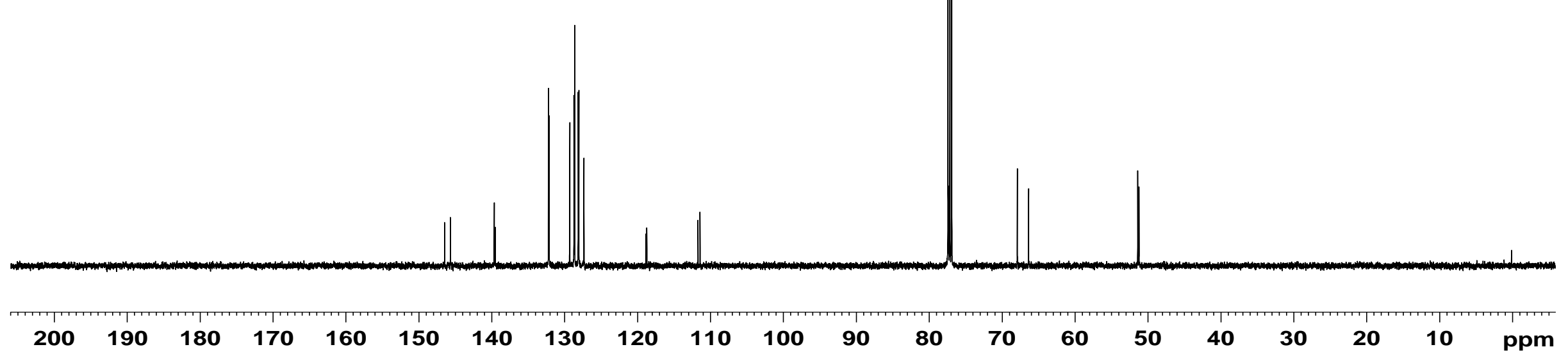

Figure S62. ${ }^{13} \mathrm{C}$ NMR spectra of $4 \mathbf{b}\left(\mathrm{CDCl}_{3}, 125 \mathrm{M}\right)$. 

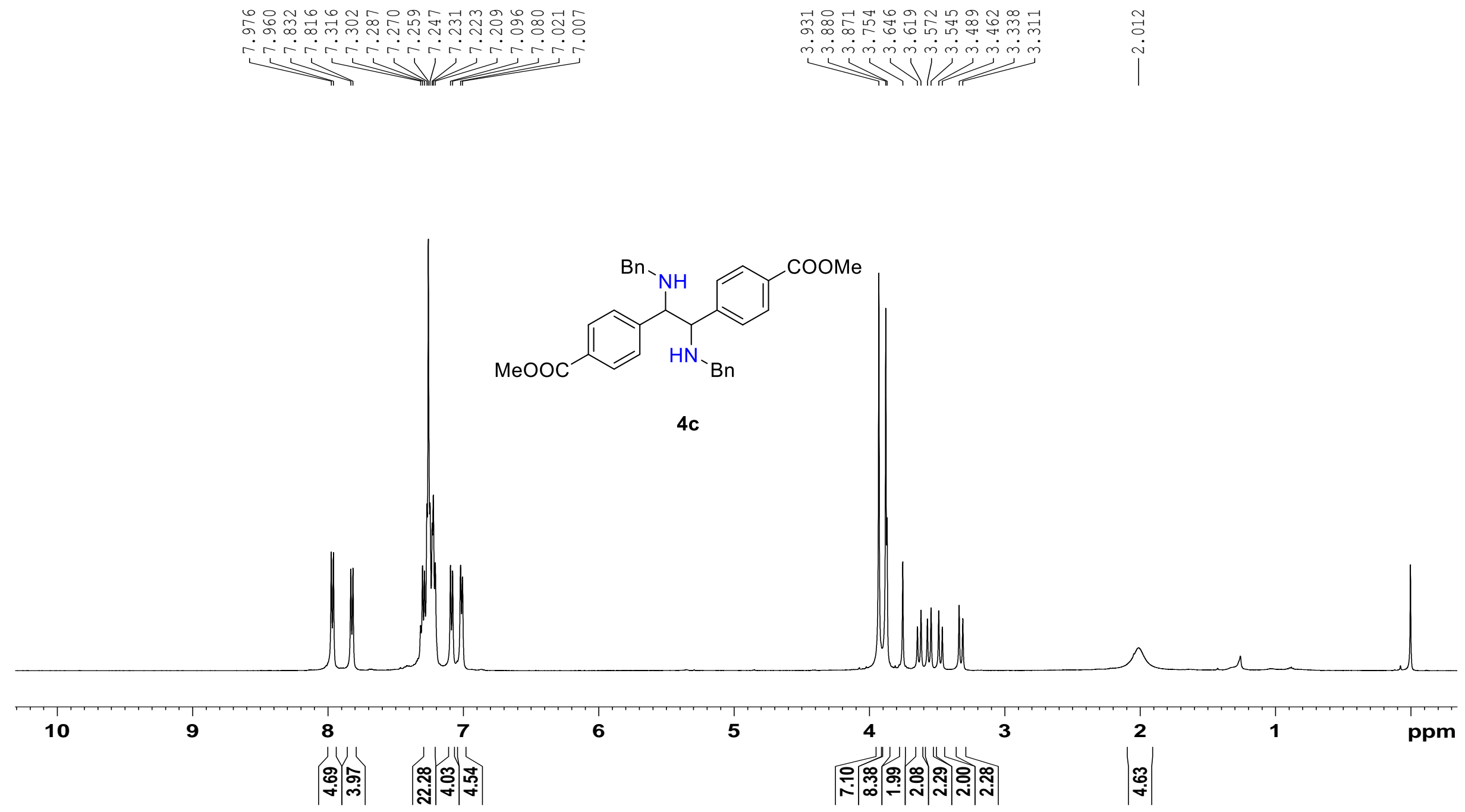

Figure S63. ${ }^{1} \mathrm{H}$ NMR spectra of $\mathbf{4 c}\left(\mathrm{CDCl}_{3}, 500 \mathrm{M}\right)$. 

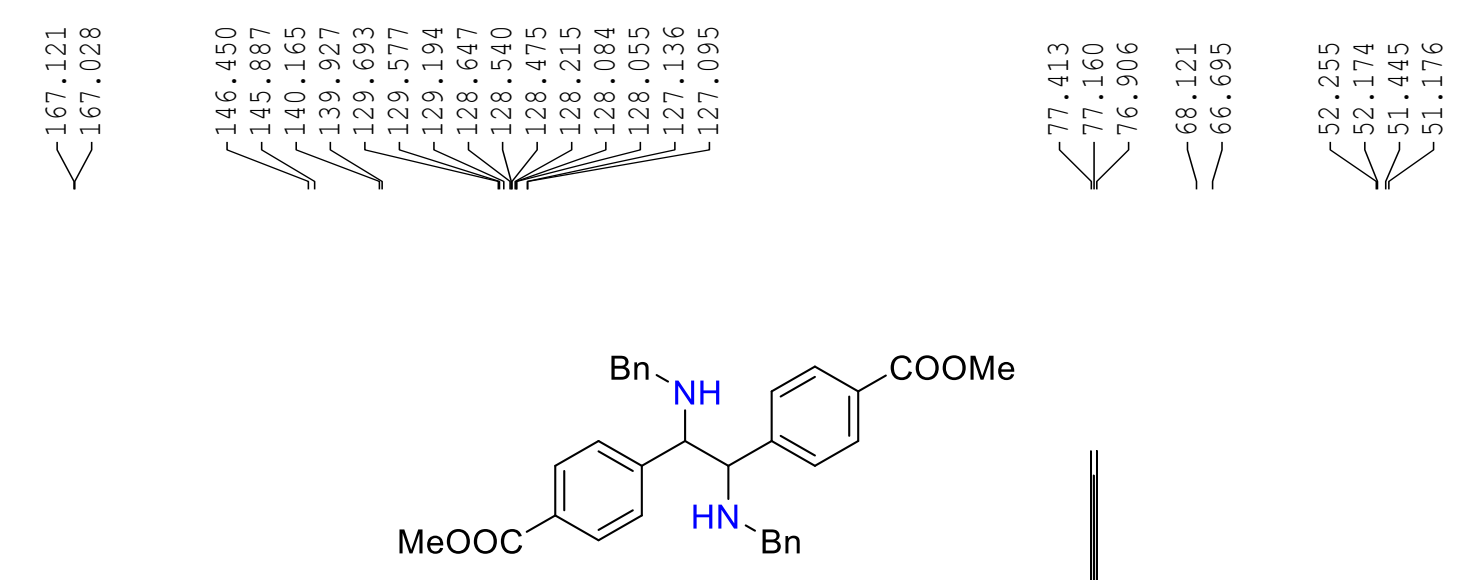

4c

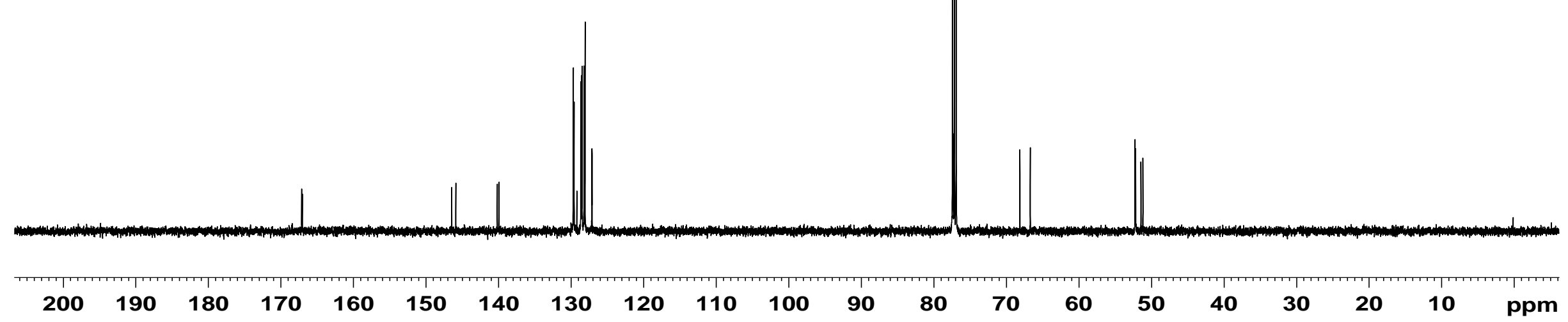

Figure S64. ${ }^{13} \mathrm{C}$ NMR spectra of $4 \mathbf{c}\left(\mathrm{CDCl}_{3}, 125 \mathrm{M}\right)$. 

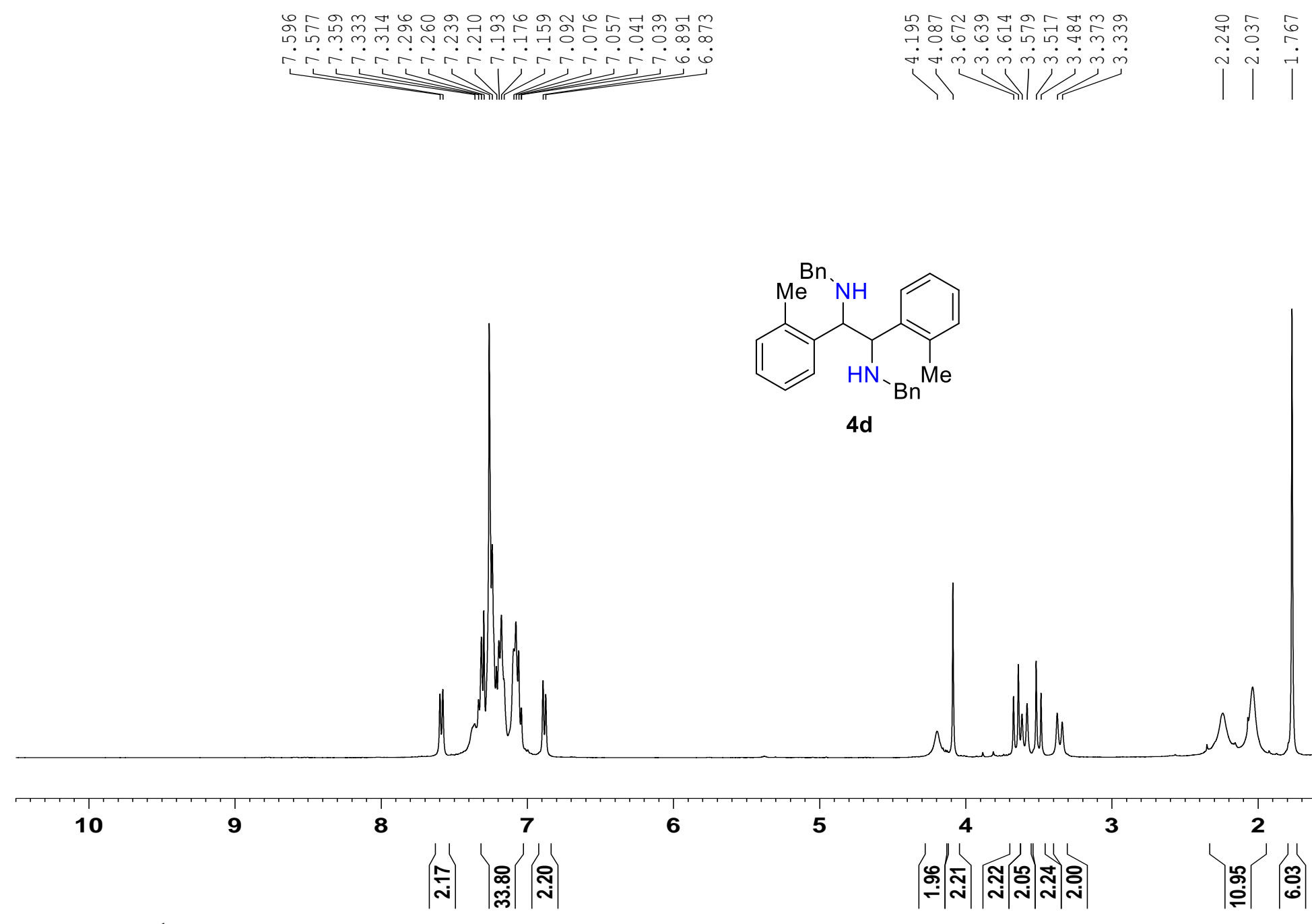

4d

Figure S65. ${ }^{1} \mathrm{H}$ NMR spectra of $\mathbf{4 d}\left(\mathrm{CDCl}_{3}, 400 \mathrm{M}\right)$. 

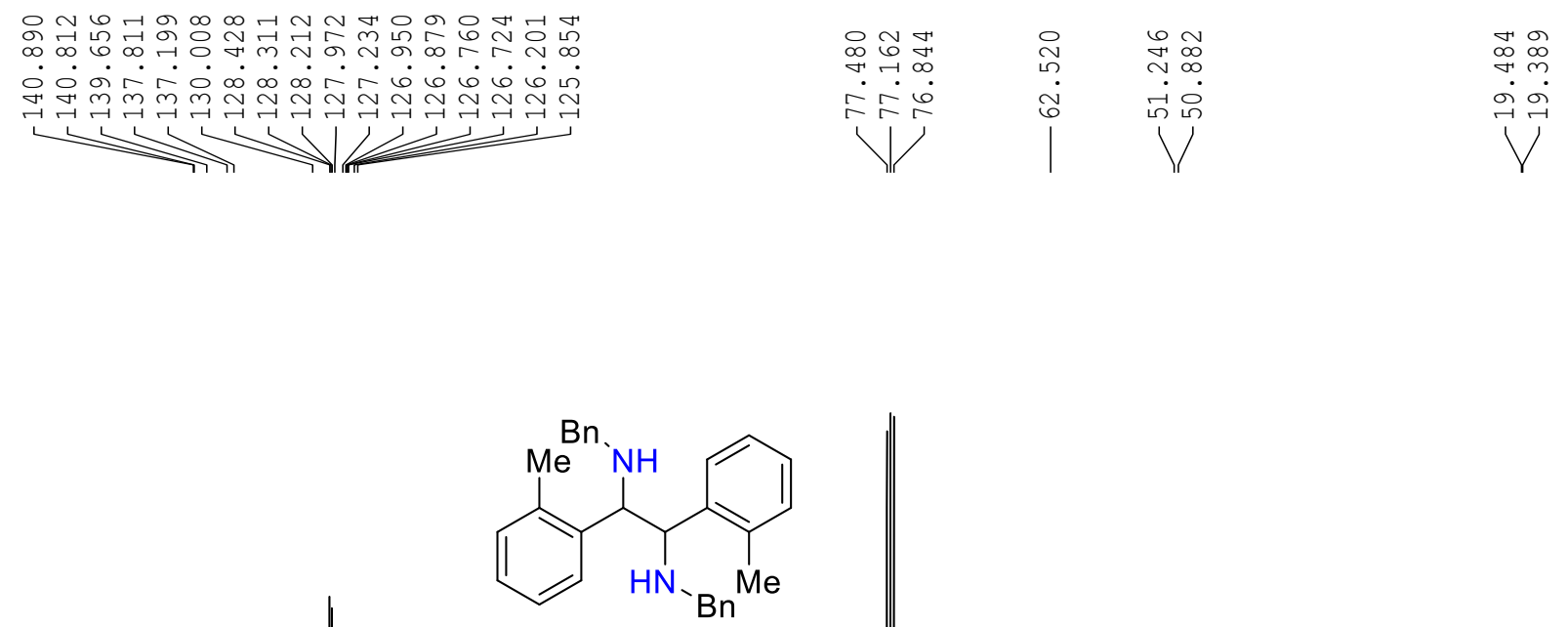

4d

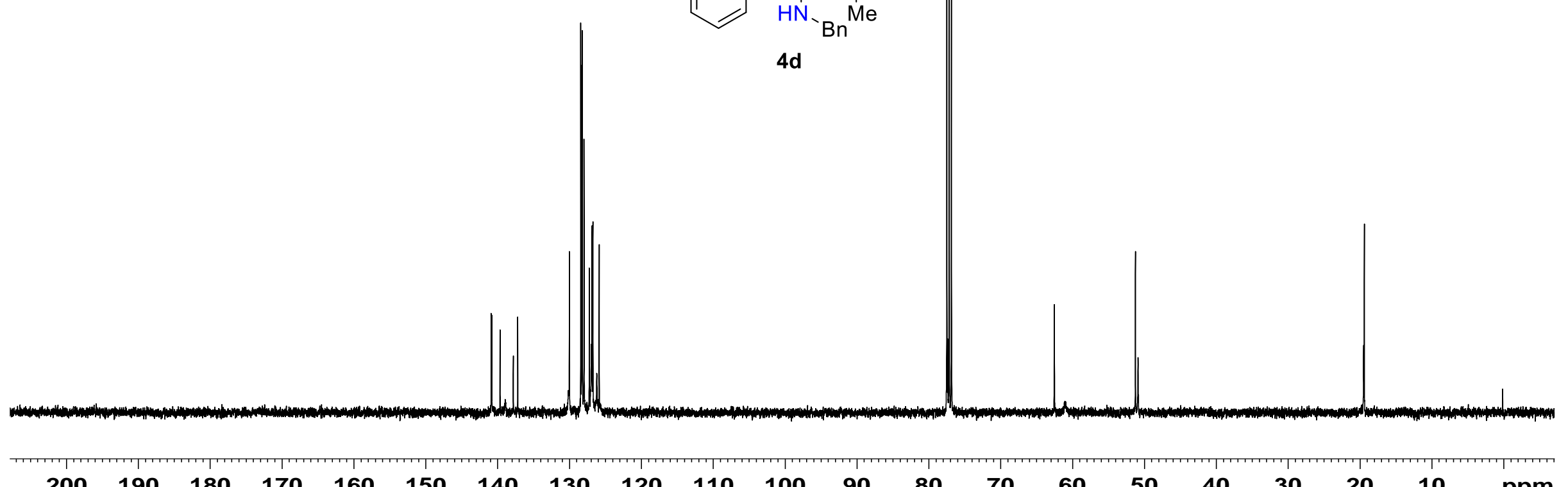

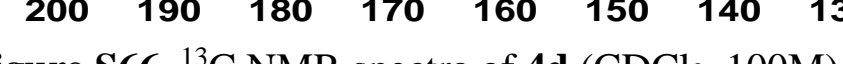




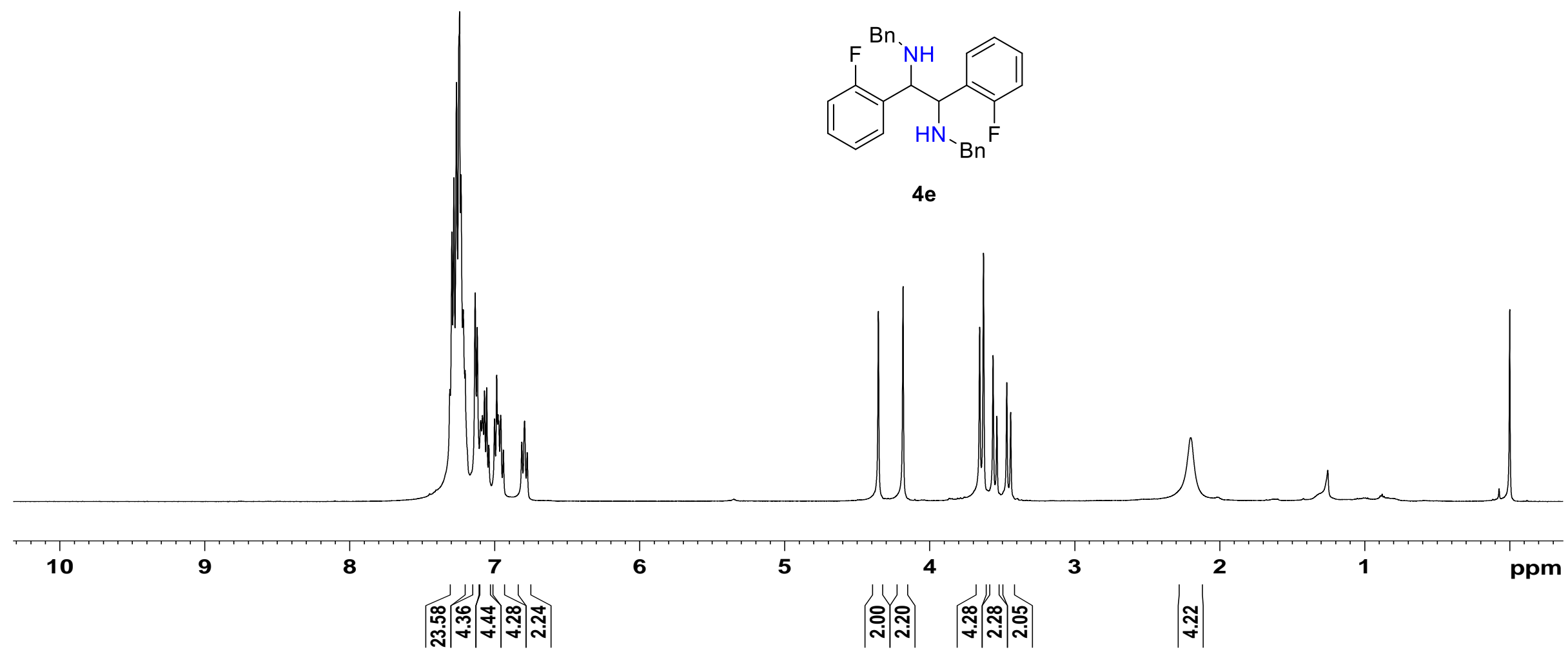

$4 e$

Figure S67. ${ }^{1} \mathrm{H}$ NMR spectra of $4 \mathbf{e}\left(\mathrm{CDCl}_{3}, 500 \mathrm{M}\right)$. 

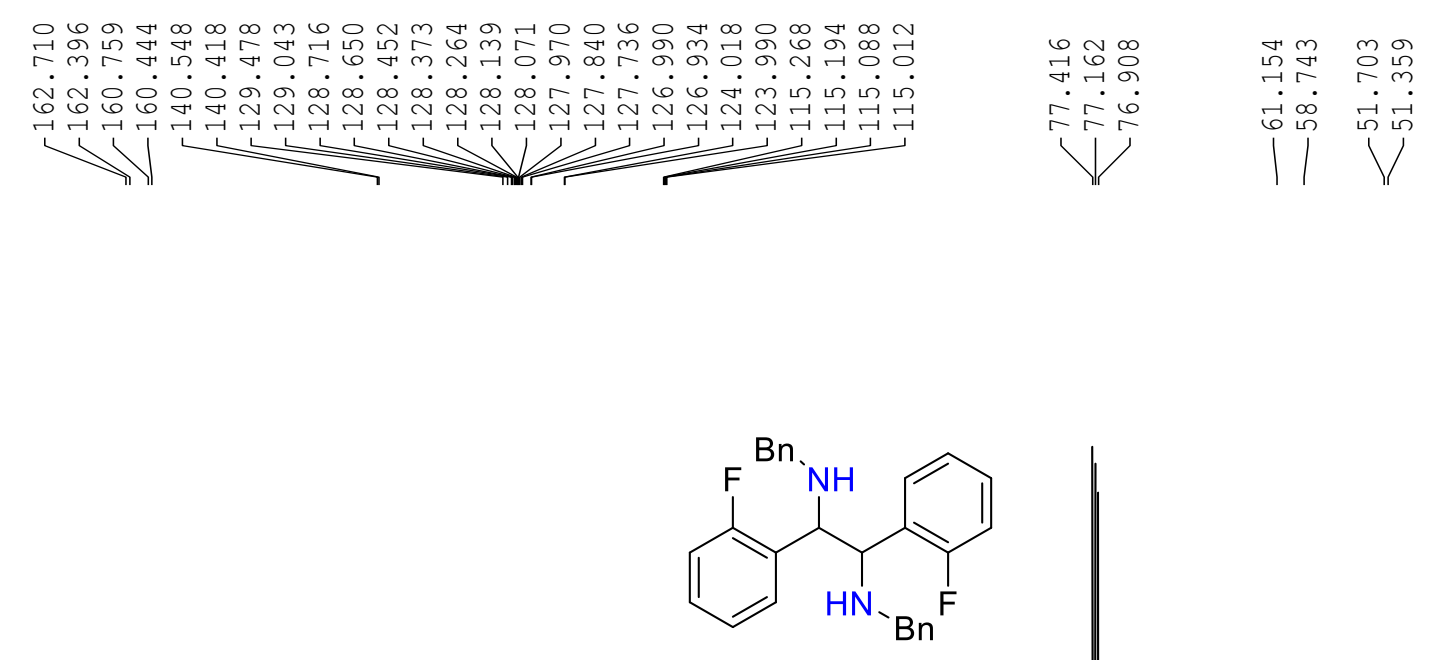

$4 e$

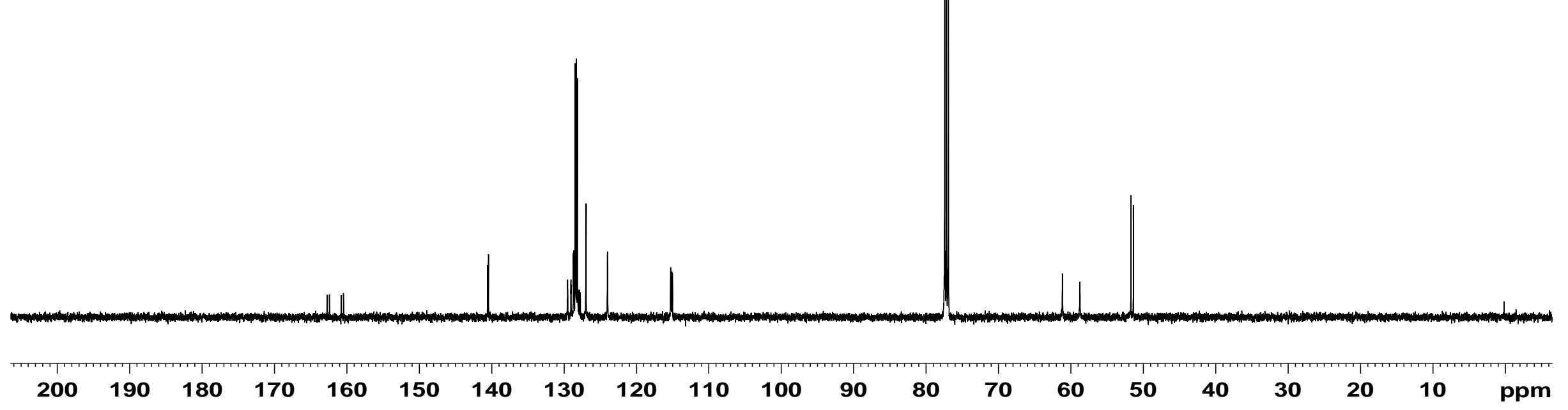

Figure S68. ${ }^{13} \mathrm{C}$ NMR spectra of $4 \mathbf{e}\left(\mathrm{CDCl}_{3}, 125 \mathrm{M}\right)$. 


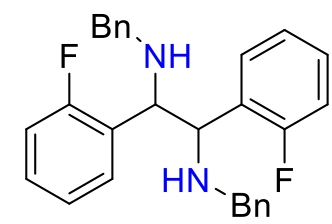

$4 e$

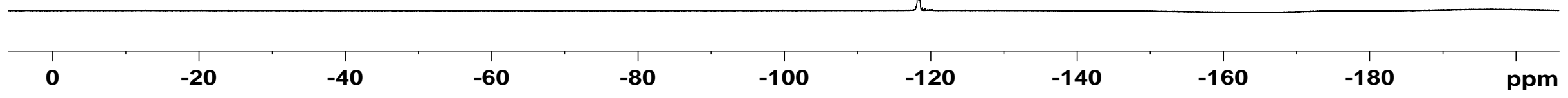

Figure S69. ${ }^{19} \mathrm{~F}$ NMR spectra of $4 \mathbf{e}\left(\mathrm{CDCl}_{3}, 471 \mathrm{M}\right)$. 

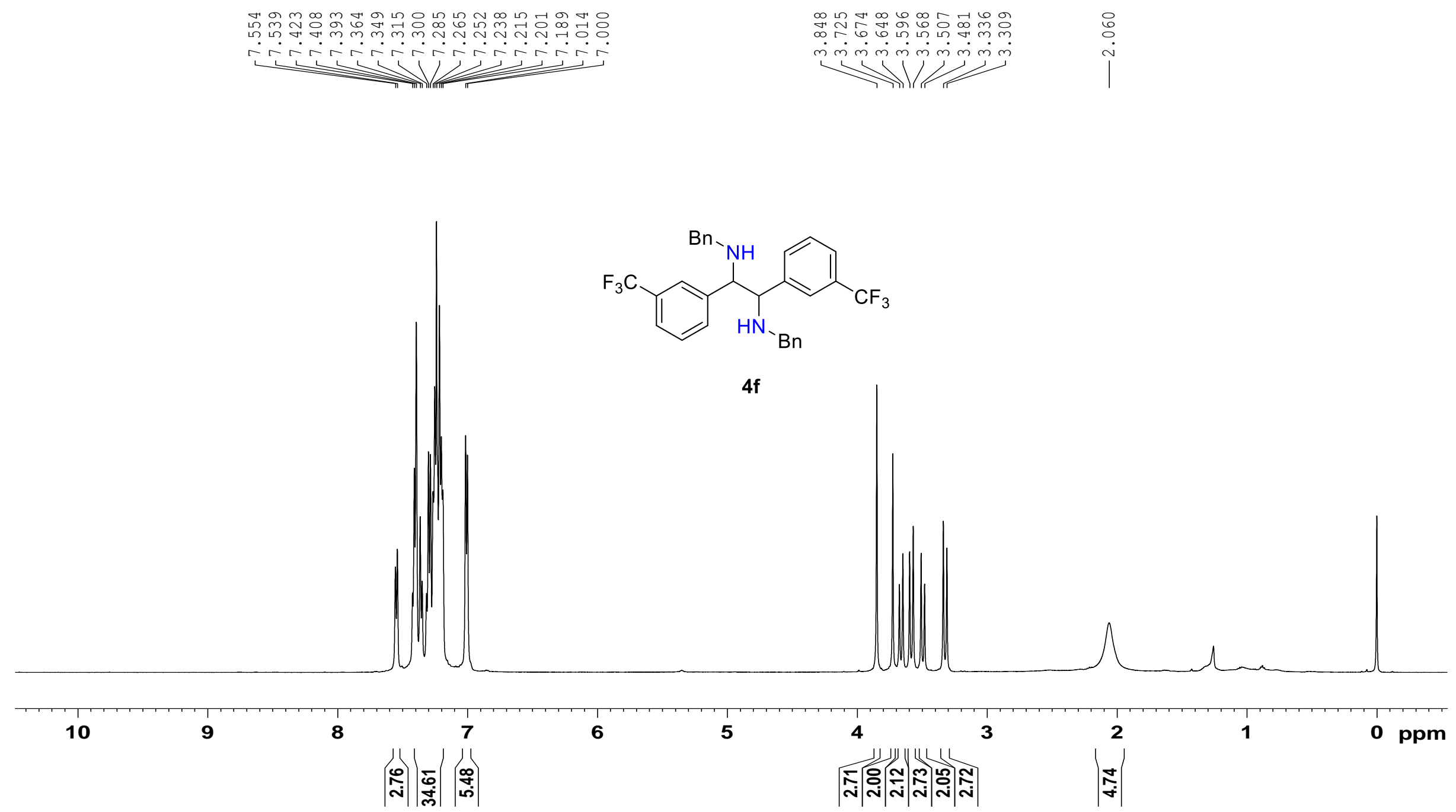

Figure S70. ${ }^{1} \mathrm{H}$ NMR spectra of $\mathbf{4 f}\left(\mathrm{CDCl}_{3}, 500 \mathrm{M}\right)$. 


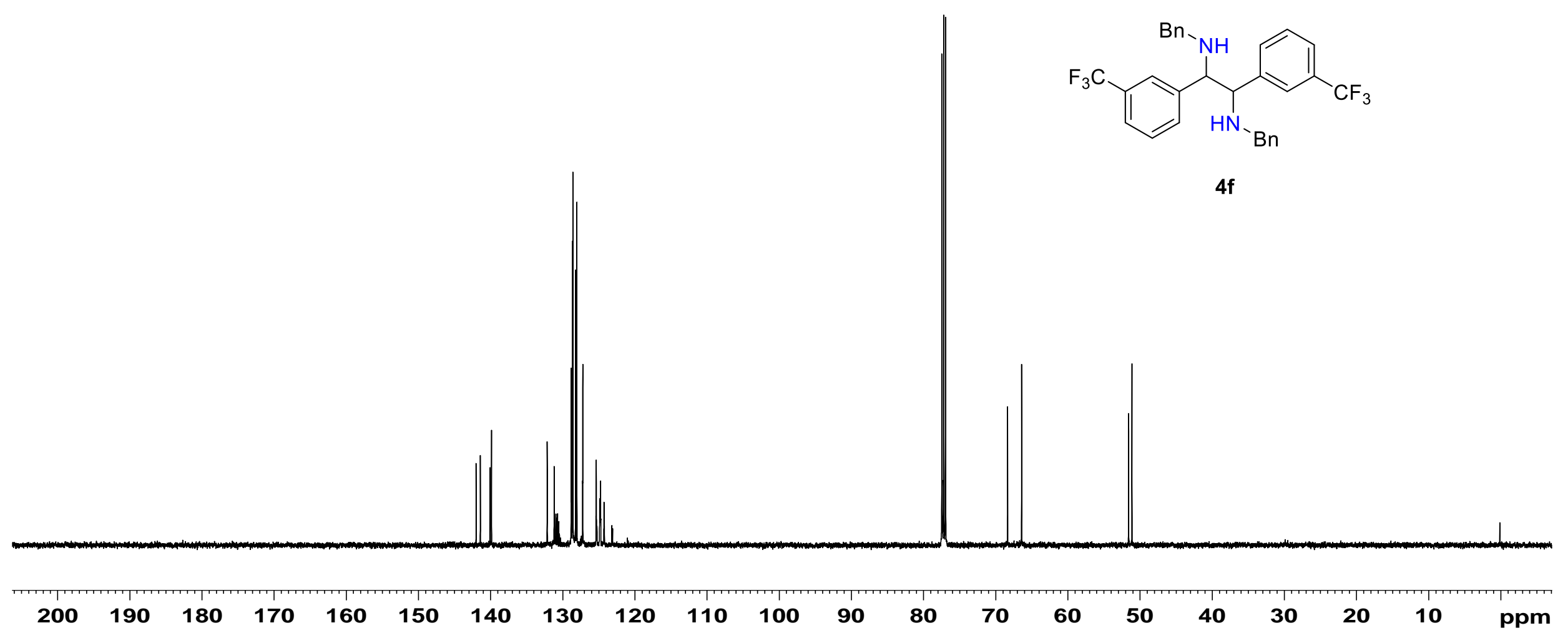

Figure S71. ${ }^{13} \mathrm{C}$ NMR spectra of $4 \mathbf{f}\left(\mathrm{CDCl}_{3}, 125 \mathrm{M}\right)$. 


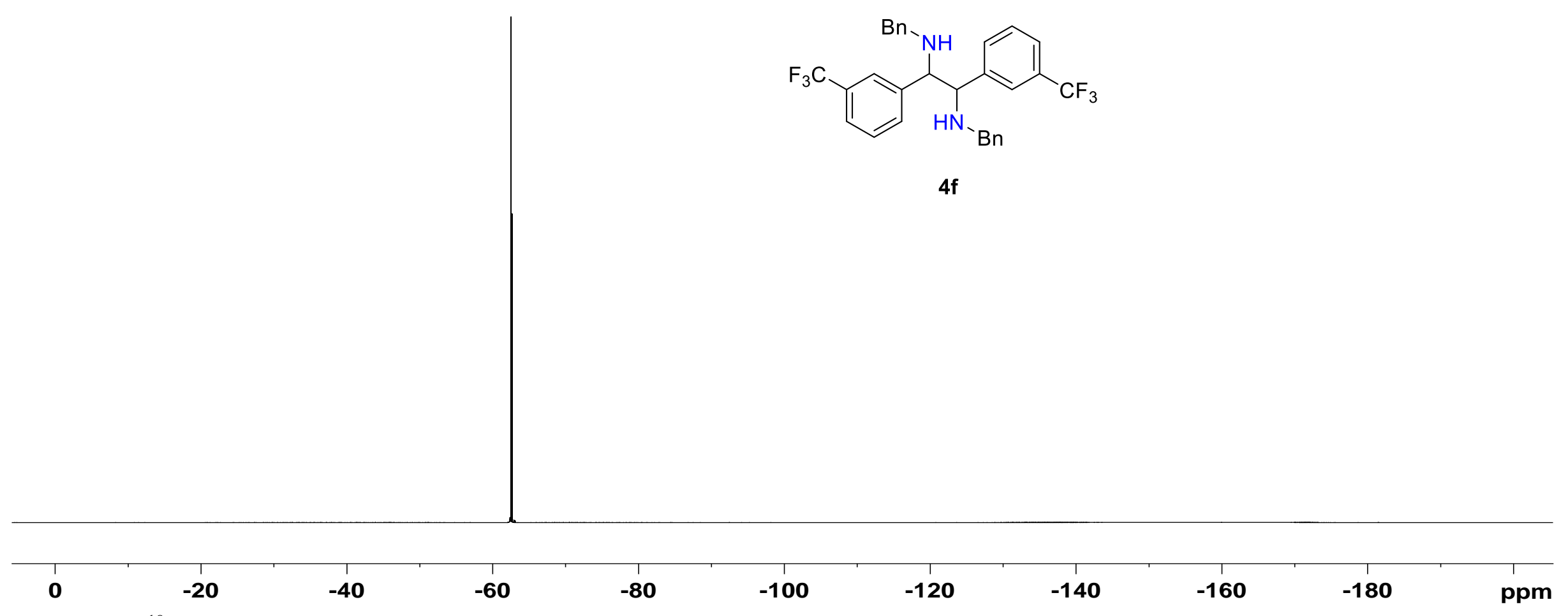

Figure S72. ${ }^{19} \mathrm{~F}$ NMR spectra of $\mathbf{4 f}\left(\mathrm{CDCl}_{3}, 471 \mathrm{M}\right)$. 

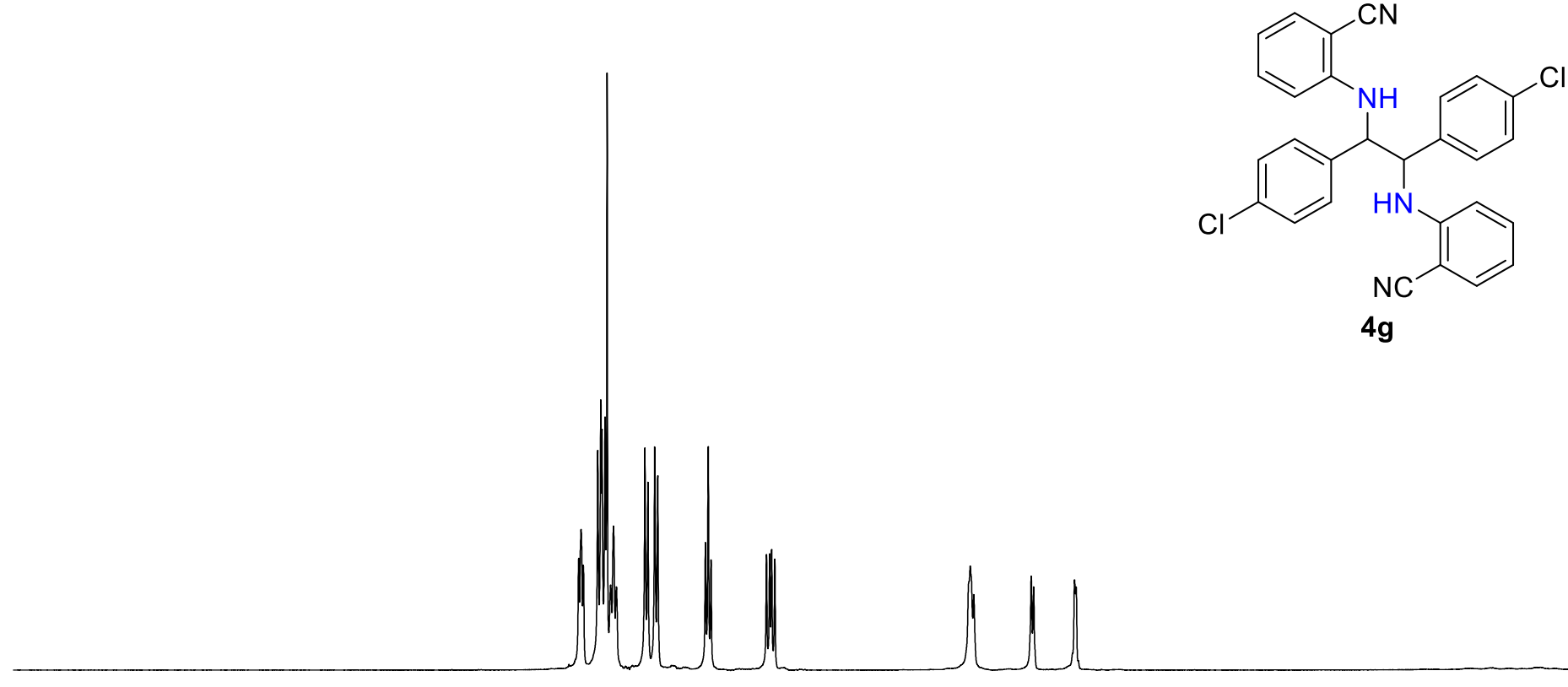

10

9

8

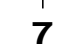

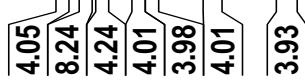

6

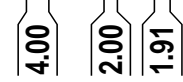

Figure S73. ${ }^{1} \mathrm{H}$ NMR spectra of $\mathbf{4 g}\left(\mathrm{CDCl}_{3}, 500 \mathrm{M}\right)$. 

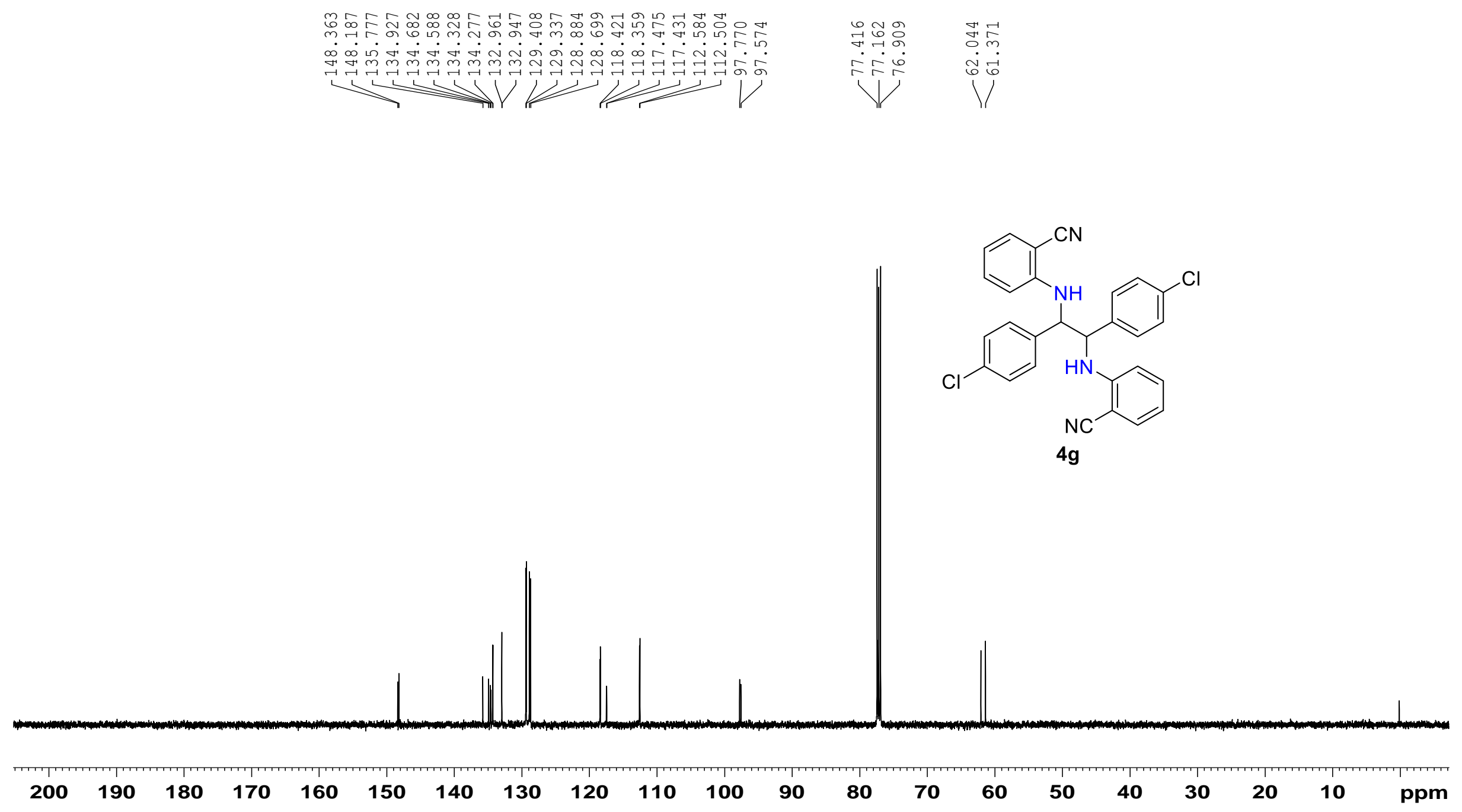

Figure S74. ${ }^{13} \mathrm{C}$ NMR spectra of $4 \mathrm{~g}\left(\mathrm{CDCl}_{3}, 125 \mathrm{M}\right)$. 

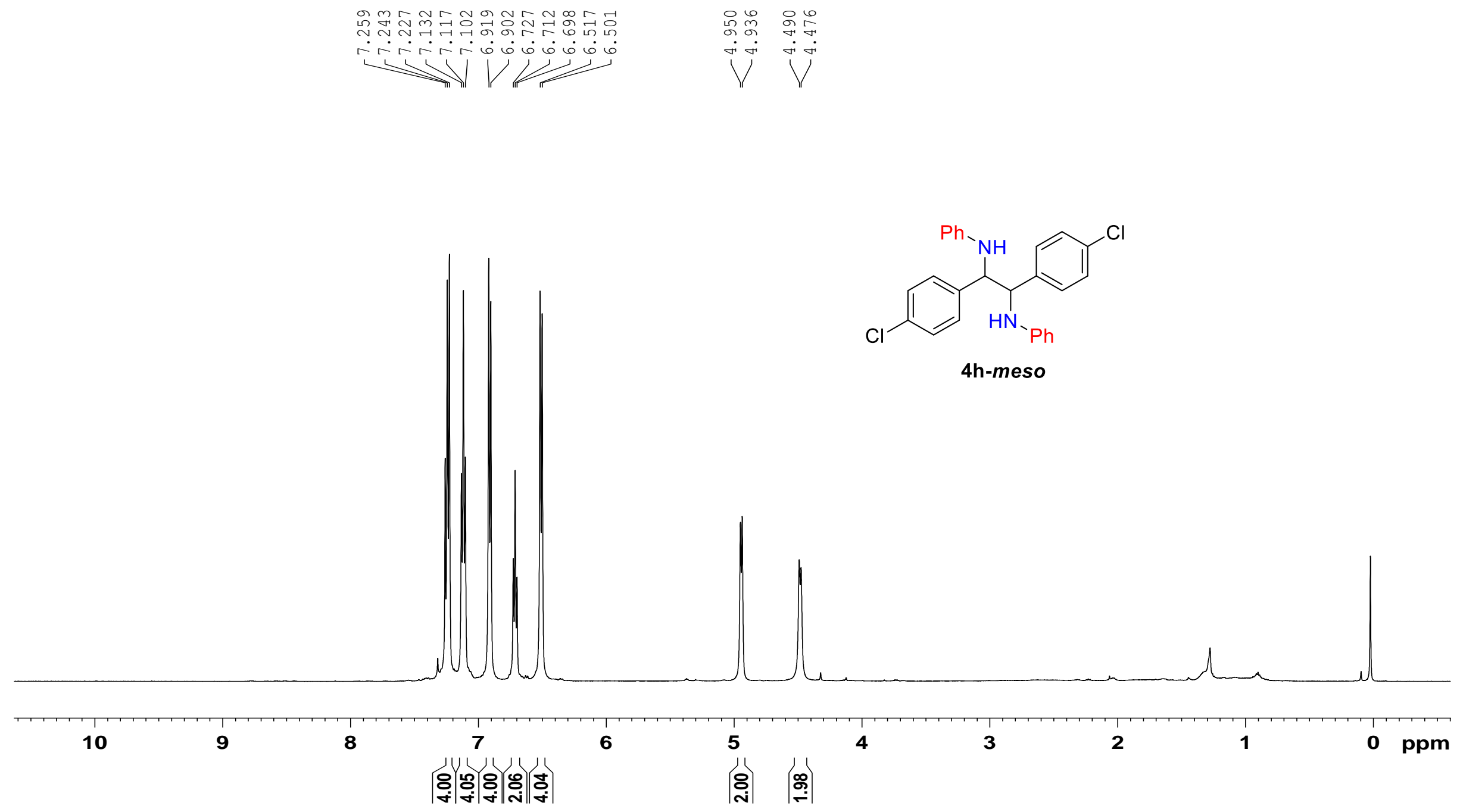

Figure S75. ${ }^{1} \mathrm{H}$ NMR spectra of $\mathbf{4 h}-$ meso $\left(\mathrm{CDCl}_{3}, 500 \mathrm{M}\right)$. 

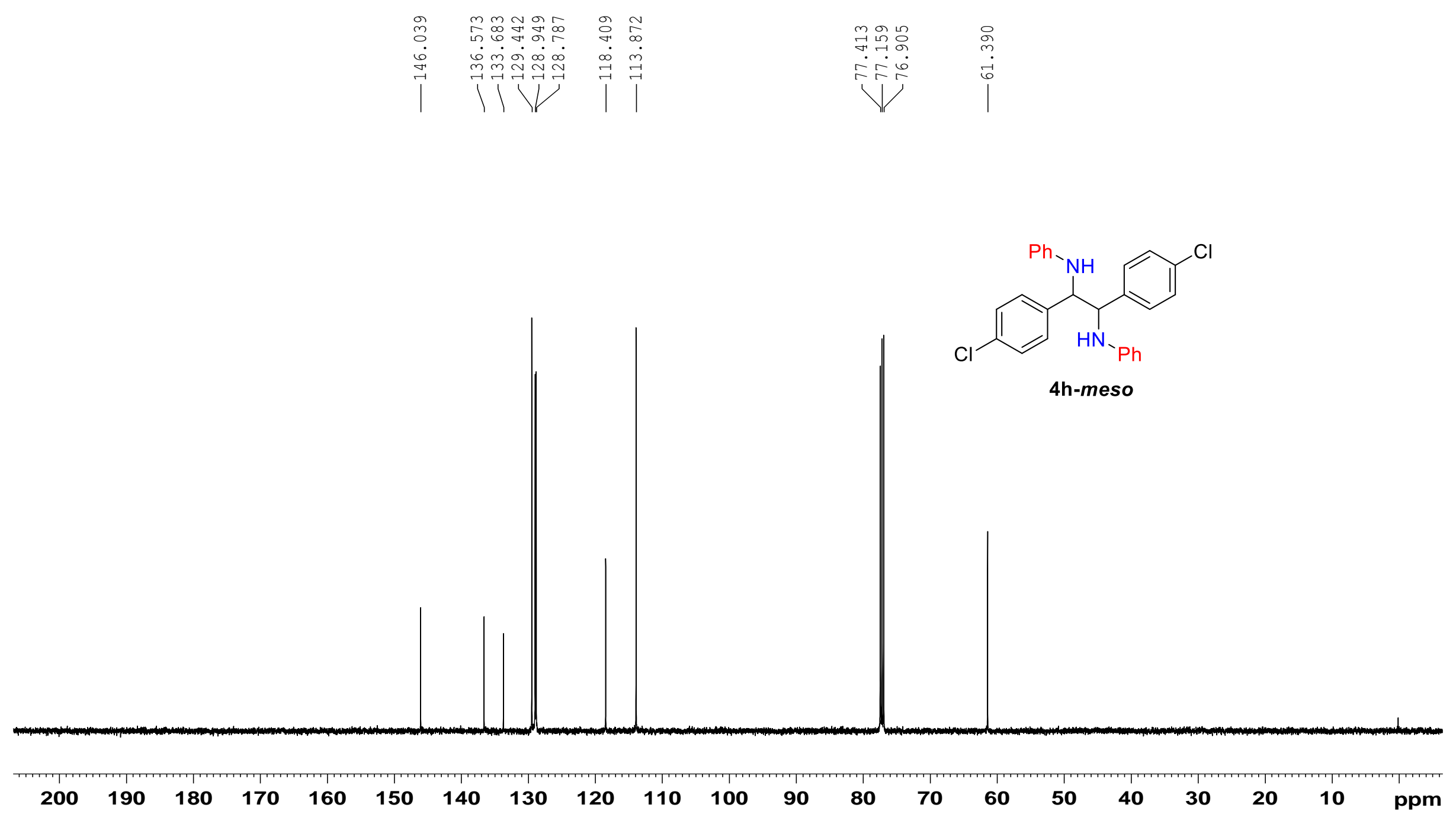

Figure S76. ${ }^{13} \mathrm{C}$ NMR spectra of $\mathbf{4 h}-$ meso $\left(\mathrm{CDCl}_{3}, 125 \mathrm{M}\right)$. 

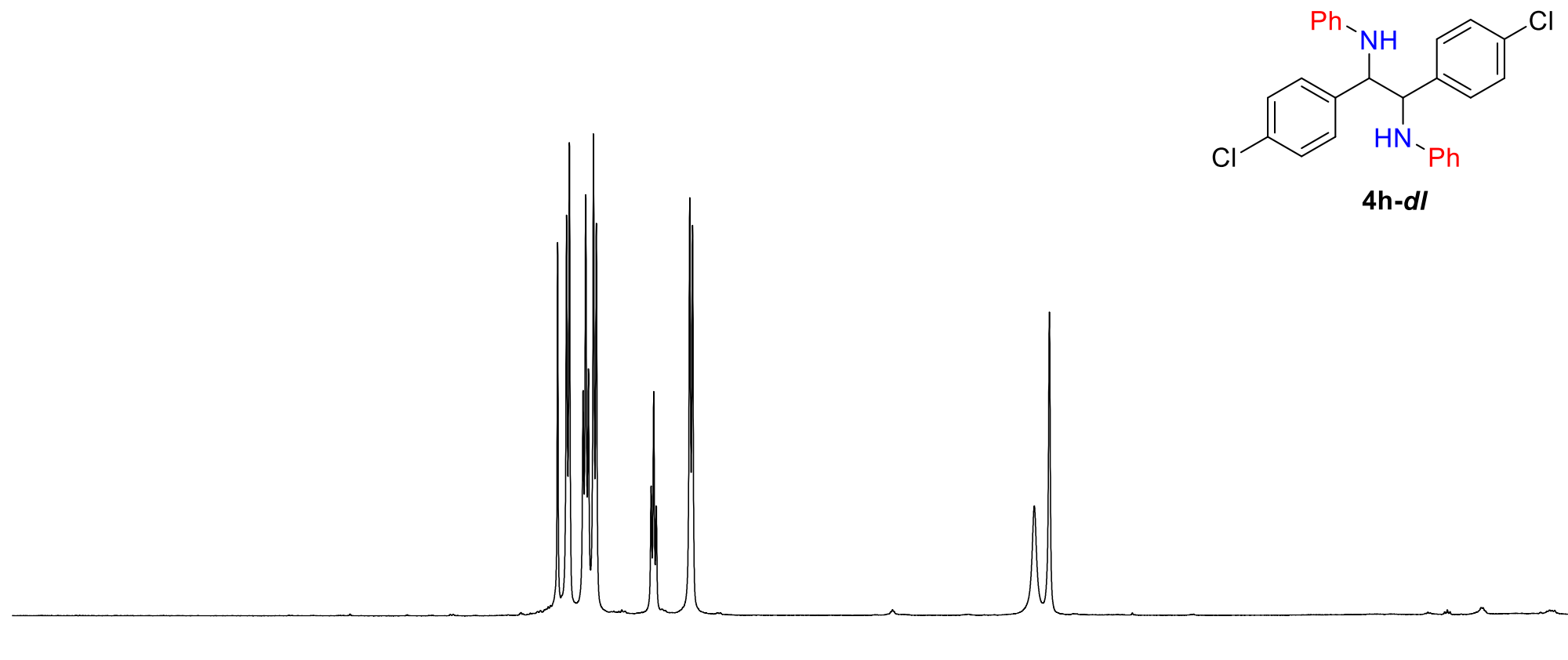

Figure S77. ${ }^{1} \mathrm{H}$ NMR spectra of $\mathbf{4 h}-\boldsymbol{d l}\left(\mathrm{CDCl}_{3}, 500 \mathrm{M}\right)$. 


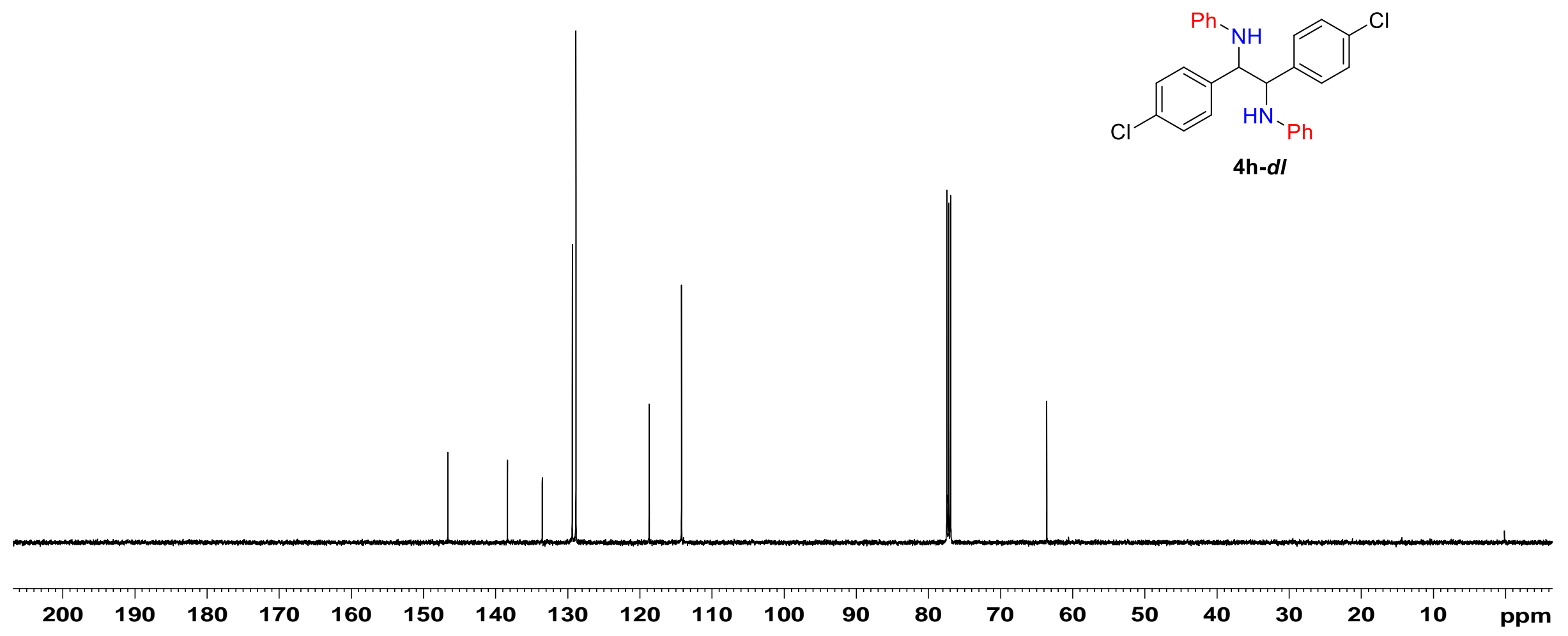

Figure S78. ${ }^{13} \mathrm{C}$ NMR spectra of $\mathbf{4 h}-\boldsymbol{d l}\left(\mathrm{CDCl}_{3}, 125 \mathrm{M}\right)$. 


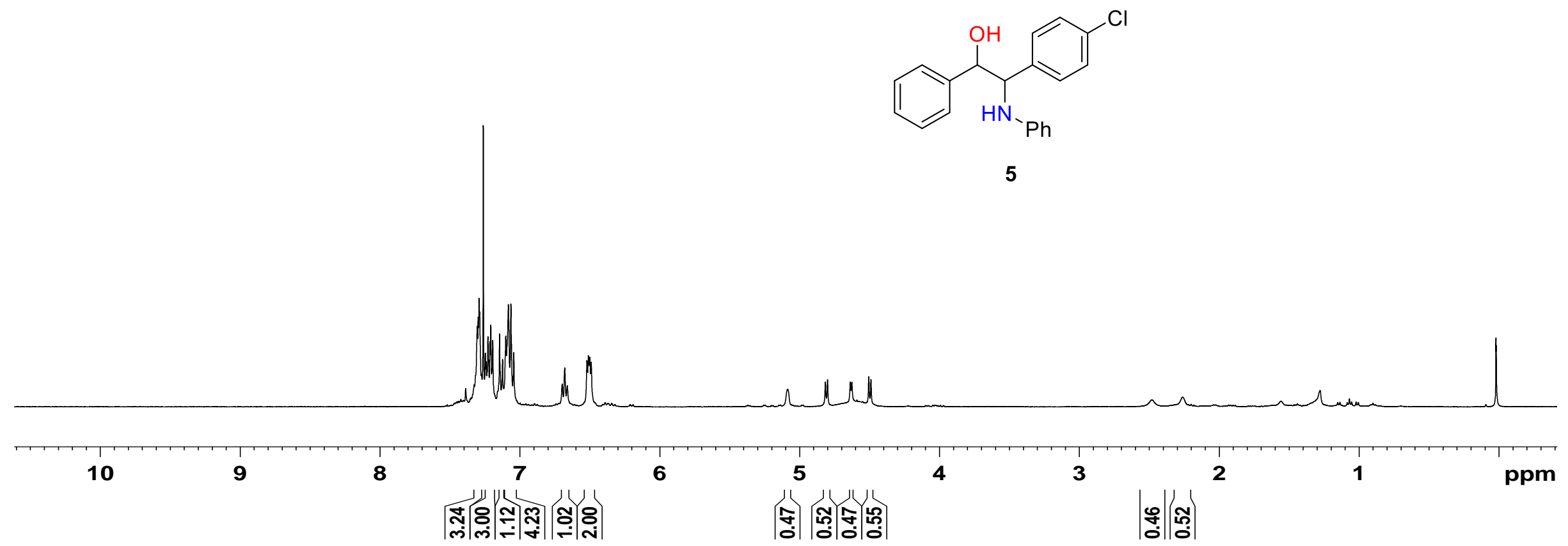

Figure S79. ${ }^{1} \mathrm{H}$ NMR spectra of $5\left(\mathrm{CDCl}_{3}, 400 \mathrm{M}\right)$. 

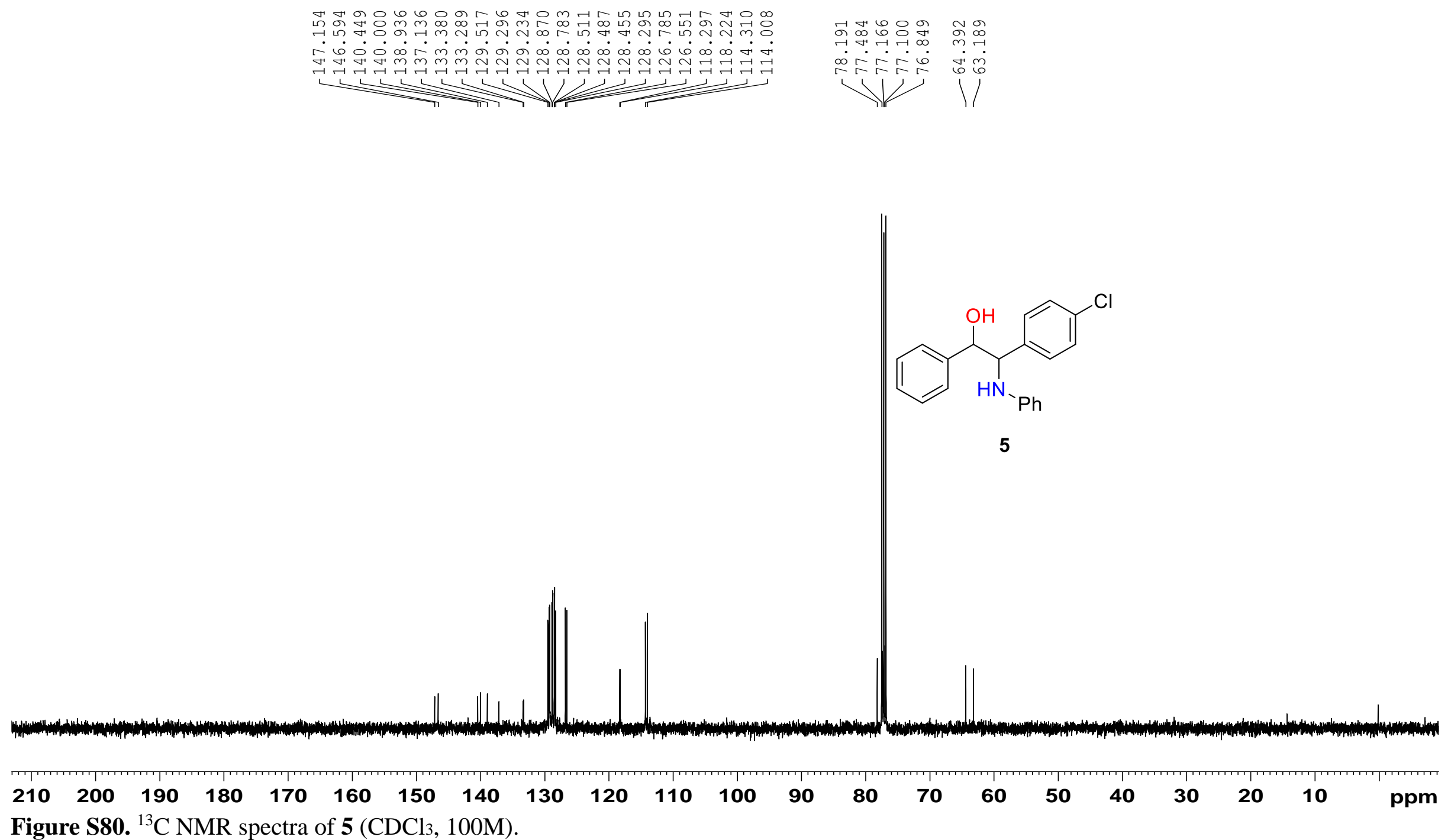